\title{
The Advanced Light Source: Technical Design
}

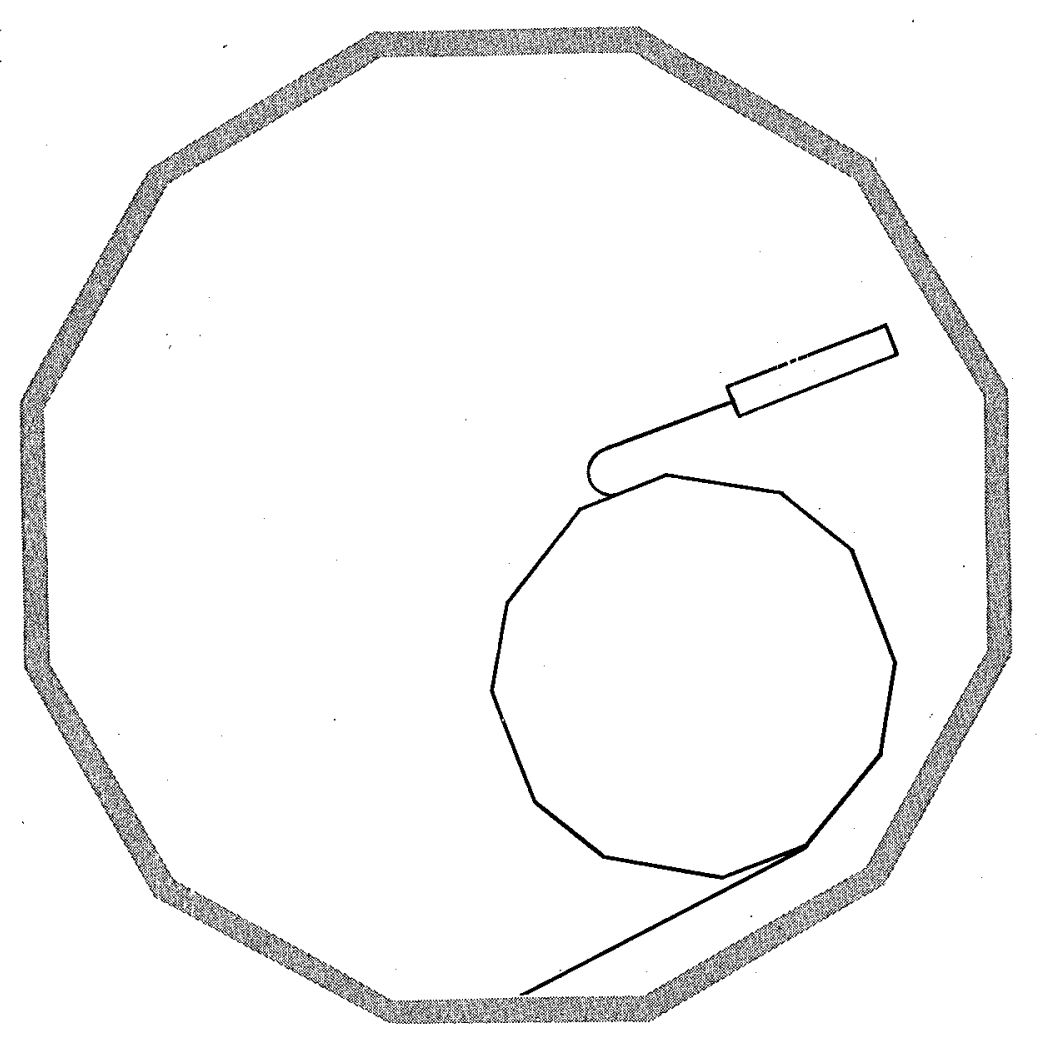

Lawrence Berkeley Laboratory

University of California

Berkeley, California 94720 
PUB-5111

Appendix A

\section{The Advanced Light Source: Technical Design}

MAY 1984

Lawrence Berkeley Laboratory

University of California

Berkeley, California 94720

This work was supported by the U.S. Department of Energy under Contract DE-AC03-76SF00098. 



\section{Contents}

Introduction to Synchrotron-Radiation Sources ...............................................................

Characteristics of Synchrotron Radiation ..................................................................

Theory of Storage Rings ............................................................................................ 5

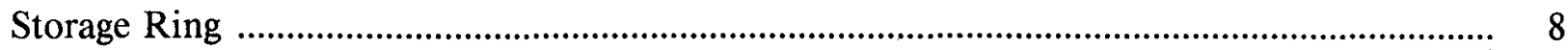

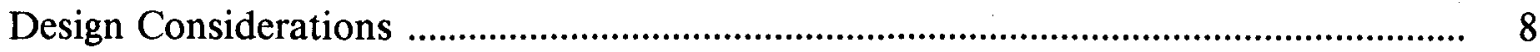

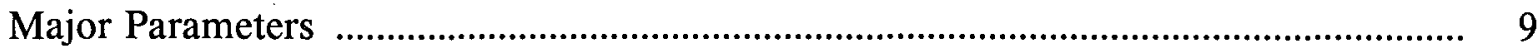

ALS Lattice ..................................................................................................... 19

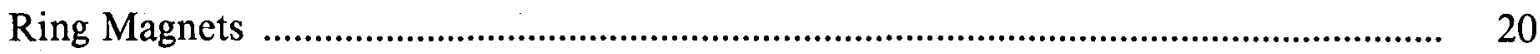

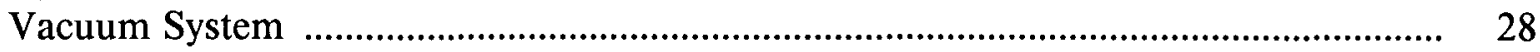

Magnet Power-Supply System ................................................................................... 31

RF System ................................................................................................... 34

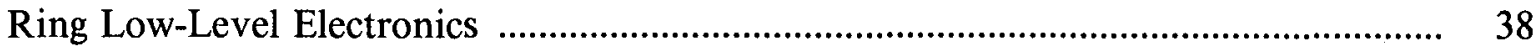

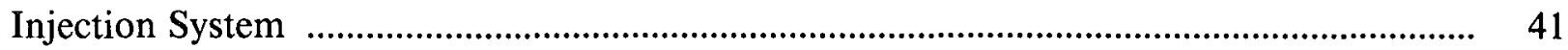

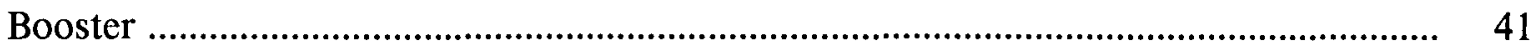

Beam Transfer System ........................................................................................... 48

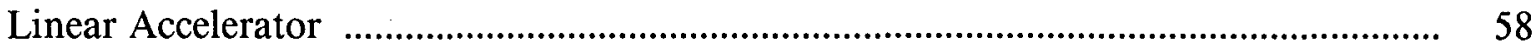

Positron Injection Option ................................................................................... $\quad 60$

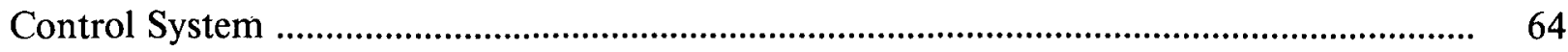

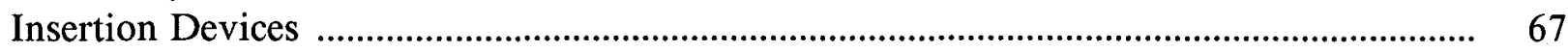

Room-Temperature Insertion Devices ................................................................... 67

Superconducting Wiggler ...................................................................................... 71

Photon Beam Lines ......................................................................................................... 74

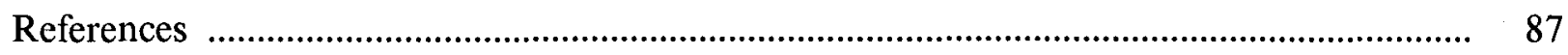




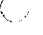




\section{Advanced Light Source}

\section{Introduction to Synchrotron-Radiation Sources}

The Advanced Light Source (ALS) is a synchrotron radiation source consisting of a $50-\mathrm{MeV}$ linear accelerator, a $1.3-\mathrm{GeV}$ "booster" synchrotron, a $1.3-\mathrm{GeV}$ electron storage ring, and a number of photon beam lines, as shown in Figure 1.

As an introduction to a detailed description of the Advanced Light Source, this section provides brief discussions on the characteristics of synchrotron radiation and on the theory of storage rings.

\section{Characteristics of Synchrotron Radiation}

Over the past several decades, the construction of particle accelerators and storage rings dedicated to high-energy physics research has led to the capability to store electron beams at high energy (up to many $\mathrm{GeV}$ ) for periods up to many hours. When a circulating electron beam is deflected by the bending magnets in a storage ring, an intense flux of electromagnetic radiation, called synchrotron radiation, is produced. Relativistic effects cause this synchrotron radiation to be emitted in a narrow cone centered about the electron-beam direction. For electron energies on the order of $1 \mathrm{GeV}$, the half-angle of this cone is typically a fraction of a milliradian. Originally, synchrotron radiation was viewed primarily as an annoyance, because of the need to compensate for the electron-beam energy loss by using a powerful radiofrequency accelerating system. However, it was soon recognized that synchrotron radiation is useful for a wide range of physical, chemical, and biological experiments, quite unrelated to the original purpose of electron storage rings. More recently, electron storage rings specifically dedicated to producing synchrotron radiation have been designed and constructed. Most storage rings in this second generation of synchrotronradiation sources are just becoming operational.

Two measures of photon beam intensity will be defined here, to permit comparisons of photon beams. First, we consider the quantity called "spectral brilliance," which is measured in the following units:

$$
\text { Spectral Brilliance }=\frac{\text { number of photons }}{\mathrm{s} \cdot(\mathrm{mm})^{2} \cdot(\mathrm{mr})^{2} \cdot(0.1 \% \text { bandwidth })}
$$




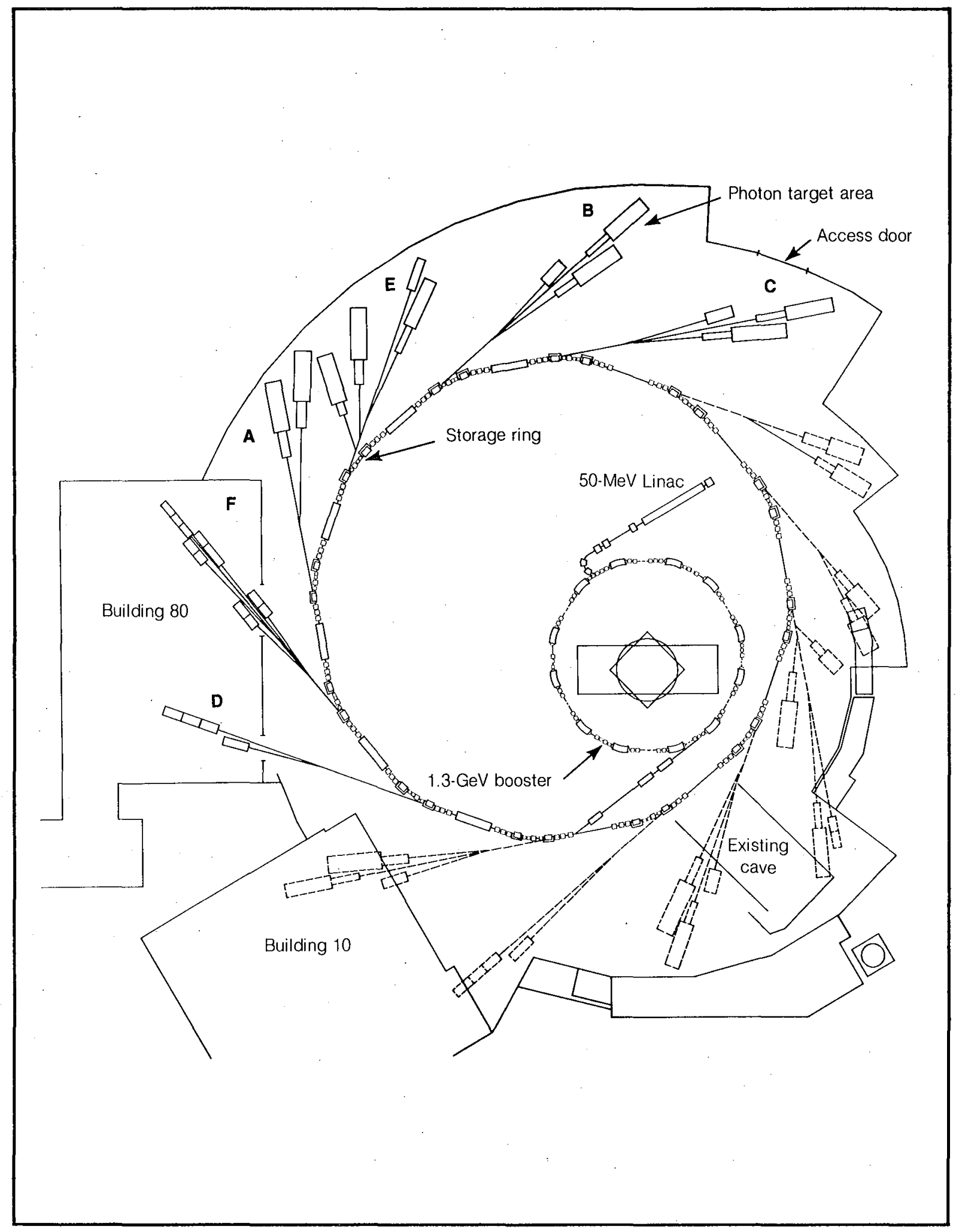

Figure 1. General plan for Advanced Light Source

XBL 833-8926 
This quantity is the spectral intensity of the radiation divided by the phase-space volume into which the radiation is emitted. (The phase-space volume is the product of the crosssectional area of the electron beam, measured in $\mathrm{mm}^{2}$, and the solid angle of the cone of radiation, measured in $\mathrm{mr}^{2}$.) A second useful measure of photon intensity is usually called "photon flux," defined as

$$
\text { Photon Flux }=\frac{\text { number of photons }}{\mathrm{s} \cdot \mathrm{mr}(\theta) \cdot(0.1 \% \text { bandwidth })} \quad, \text { for all } \psi .
$$

This quantity is the spectral brilliance integrated over the following three variables: the electron-beam width, the electron-beam height, and the vertical angle $\psi$. Note that the photon flux is still a differential quantity with respect to the horizontal angle $\theta$. For experiments that require the synchrotron radiation to originate from a small volume in phase space, spectral brilliance is a figure of merit for photon intensity. For experiments that can use photons from a large volume of phase space, the integrated quantity of photon flux is a better measure of intensity.

Storage-ring dipole bending magnets, which keep the electron beam in its approximately circular orbit, can produce useful beams of synchrotron radiation. However, special magnetic devices inserted in storage-ring straight sections can produce far more intense photon beams with greater flexibility in terms of photon beam characteristics. These magnetic devices, called "wigglers" and "undulators," are characterized by static transverse magnetic fields that alternate in direction periodically as the electrons traverse the length of the device. The distinction between wigglers and undulators is given by the dimensionless parameter

$$
\mathrm{K}=0.934 \mathrm{~B} \lambda_{\mathrm{w}}
$$

where $\mathrm{B}$ is the magnetic field (in Tesla) and $\lambda_{\mathrm{w}}$ is the spatial period (in $\mathrm{cm}$ ) of the alternations of the field. for $\mathrm{K} \gtrsim 10$, the magnetic device is called a "wiggler," and the spectral distribution of the synchrotron radiation is smooth and wide, as it is for a bending magnet. That is, in a wiggler the interference effects of the radiation are small, and the wiggler acts like a series of bending magnets whose synchrotron radiation combines to provide an intense beam. Wigglers particularly enhance photon flux. For values of $K \leqslant 1$, however, interference effects are important, and the device is called an "undulator." The synchrotron radiation from an undulator is spectrally compressed into a series of narrow peaks (see Figure 2), so undulators can provide unparalleled spectral brilliance. More detailed discussions of the radiation from wigglers and undulators are available in the literature [1].

It should be noted that the effect of a wiggler or an undulator on the operation of a storage ring is much less than that of a bending magnet of comparable strength, because of the cancellations produced by the spatially alternating fields. Therefore, one has far greater flexibility in selecting undulator and wiggler parameters than in selecting bending-magnet parameters. This flexibility is particularly useful because the energy of the synchrotronradiation photons can be controlled by selecting the strength of the magnetic field without changing the electron energy. 


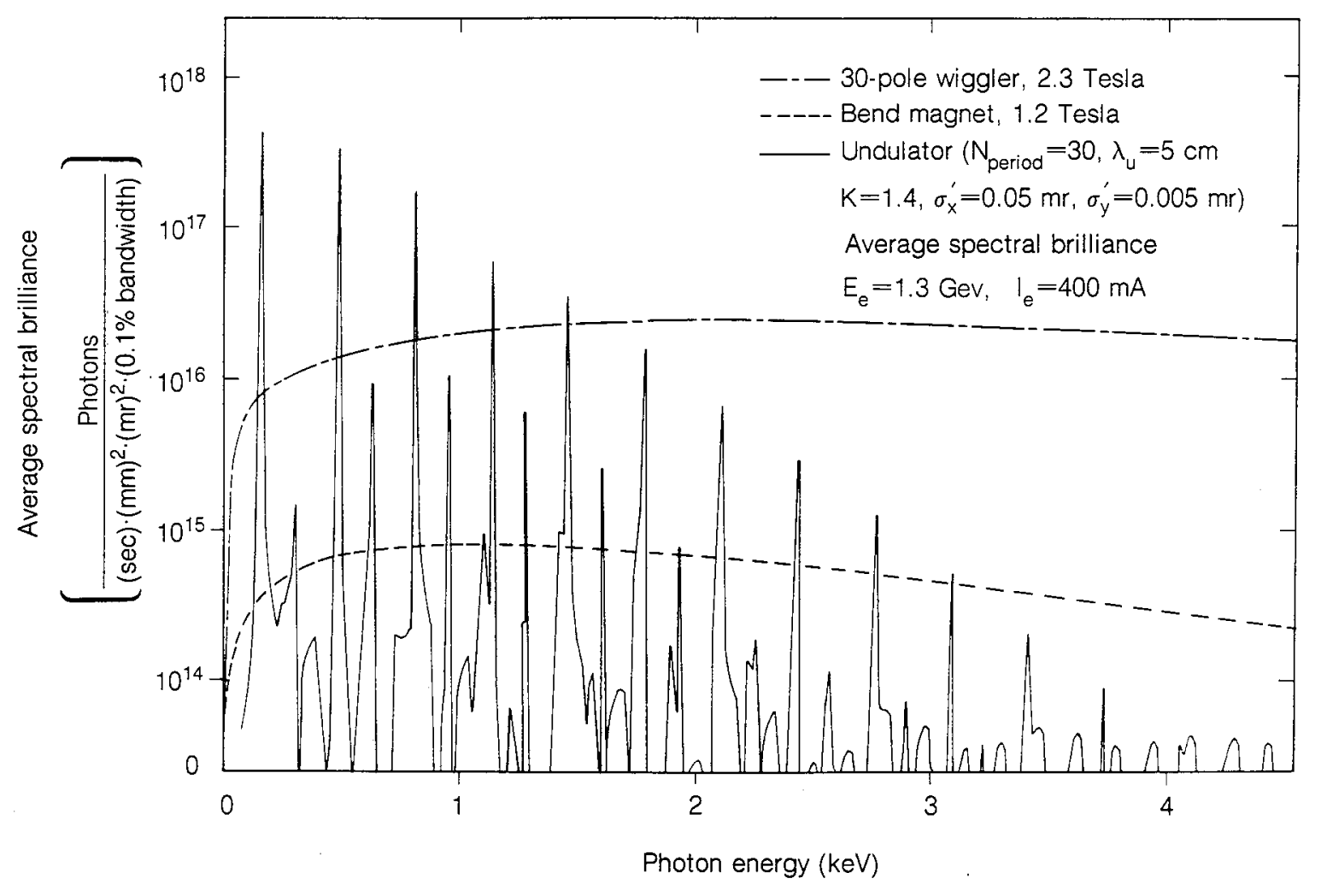

Figure 2. Spectral brilliance of bend magnet, wiggler, and undulator. 


\section{Theory of Storage Rings}

This subsection will present a brief discussion of the theory of electron storage rings. Some basic concepts concerning transverse and longitudinal particle dynamics will be introduced, and some important beam-loss mechanisms will be discussed.

The periodic arrangement of bending and focusing magnets, separated by field-free drift regions, is called the "lattice" of a storage ring. From the viewpoint of particle dynamics, this lattice and the radiofrequency (rf) accelerating structure are the fundamental components of a ring $[2,3]$.

The transverse motion of particles may best be described in terms of deviations from the "equilibrium orbit." This equilibrium orbit is the unique periodic solution of the equations of motion for a particle with the nominal momentum, $\mathrm{p}_{0}$. It has the property of being closed on itself and is characterized by a local curvature $\Omega(\mathrm{s})=1 / \rho(\mathrm{s})$, where $\mathrm{s}$ is the distance measured along the orbit. A general particle trajectory may be described by $\mathrm{x}(\mathrm{s})$ and $y(s)$, the horizontal and vertical distances from the equilibrium orbit. For a particle with momentum $p=p_{0}+\Delta p$, the equations of motion, to first order, are as follows:

$$
\begin{gathered}
\frac{\mathrm{d}^{2} \mathrm{x}}{\mathrm{ds}^{2}}+\mathrm{K}_{\mathrm{x}}(\mathrm{s}) \cdot \mathrm{x}=\frac{1}{\rho(\mathrm{s})} \frac{\Delta \mathrm{p}}{\mathrm{p}_{\mathrm{o}}} \\
\frac{\mathrm{d}^{2} \mathrm{y}}{\mathrm{ds}^{2}}+\mathrm{K}_{\mathrm{y}}(\mathrm{s}) \cdot \mathrm{y}=0
\end{gathered}
$$

The right-hand side of the second equation is zero for accelerator designs in which the beam bending magnets deflect the beam only in the horizontal plane. The functions $\mathrm{K}_{\mathrm{x}}$ and $\mathrm{K}_{\mathrm{y}}$ are determined by the lattice elements, and they are usually piecewise constant.

The "pseudo-harmonic" solutions to these equations of motion are given in terms of the "betatron oscillations" of the particle trajectories. For a particle with nominal momentum $\mathrm{p}_{\mathrm{o}}$, a trajectory in either plane may be described in terms of oscillations whose amplitude depends on the initial conditions and is proportional to $\beta^{1 / 2}$, where $\beta(\mathrm{s})$, the CourantSnyder "beta function," is a positive function that depends on the lattice configuration and has the same periodicity as the lattice. This beta function has units of length and is readily calculated using computer codes. The phase of the betatron oscillations is given by

$$
\psi(\mathrm{s})=\int_{0}^{\mathrm{s}} \frac{\mathrm{ds}}{\beta(\mathrm{s})}
$$

The trajectories of off-momentum particles exhibit an additional deviation from the equilibrium orbit in the horizontal plane, and this offset is given by

$$
\mathrm{x}_{\mathrm{p}}=\eta(\mathrm{s}) \frac{\Delta \mathrm{p}}{\mathrm{p}_{\mathrm{o}}}
$$

where the dispersion function $\eta(\mathrm{s})$ depends on the lattice configuration and is periodic in $\mathbf{s}$.

The "tune" of an accelerator, i.e., the number of betatron periods corresponding to the accelerator circumference $C$, is given by 


$$
\nu=\frac{1}{2 \pi} \int_{0}^{\mathrm{C}} \frac{\mathrm{ds}}{\beta(\mathrm{s})}
$$

The electron beam in a storage ring is normally characterized by a Gaussian density distribution in both transverse directions, so the density distribution may be conveniently described by a standard-deviation width $\sigma$. In terms of the lattice functions $\beta_{\mathrm{x}}$ and $\beta_{\mathrm{y}}$, the beam emittances are defined as

$$
\epsilon_{\mathrm{x}}=\frac{\sigma_{\mathrm{x}}^{2}(\mathrm{~s})}{\beta_{\mathrm{x}}(\mathrm{s})} \text { and } \epsilon_{\mathrm{y}}=\frac{\sigma_{\mathrm{y}}^{2}(\mathrm{~s})}{\beta_{\mathrm{y}}(\mathrm{s})}
$$

The longitudinal motion of an electron is dominated by a balance between the energy loss from synchrotron radiation and the compensating energy gain from the radiofrequency accelerating system. The time-averaged synchrotron-radiation power loss per electron is given by

$$
P_{\gamma}=\frac{2}{3} \frac{r_{0} c}{\left(m_{0} c^{2}\right)^{3}} \frac{E^{4}}{\rho^{2}}
$$

where $E$ is the electron energy and $r_{0}$ is the classical electron radius. The energy loss in one turn is then

$$
\mathrm{U}_{\mathrm{o}}=\mathrm{P}_{\gamma} \mathrm{T}
$$

where $T$ is the transit time for one turn. This energy loss is compensated by the rf acceleration voltage

$$
V(t)=V_{0} \sin \left(h \omega_{0} t\right)
$$

where $h$ is an integer (the "harmonic number") and $\omega_{0}$ is the revolution frequency in radians per second of a particle with $p=p_{0}$. If $\mathrm{eV}_{\mathrm{o}}>\mathrm{U}_{\mathrm{o}}$, the electron beam can be captured in $h$ stable bunches around the circumference of the storage ring.

Synchrotron radiation is emitted in a forward cone centered about the electron direction of motion, so the electrons lose both longitudinal and transverse momentum. Since the rf system restores only the longitudinal component of momentum, the transverse electron motion is damped. Energy damping also occurs, because particles with different momenta follow different trajectories and experience different energy losses.

The above results are obtained by assuming a continuous energy loss from radiation. However, synchrotron radiation is actually a statistical process involving the loss of energy in discrete quanta, and there is a quantum excitation of the beam in the horizontal plane in regions with non-zero dispersion. This excitation leads to a random-walk diffusion superimposed on the damping process. The competition between damping and quantum excitation leads to equilibrium values for the energy spread and horizontal emittance of the electron beam. Emittances are larger at higher electron energies. 
In a perfectly constructed and perfectly aligned electron storage ring having no coupling between the horizontal and vertical planes, the vertical emittance of the electron beam would become negligibly small. However, in any actual machine, the unavoidable construction and alignment errors produce closed-orbit errors, horizontal-vertical coupling, and vertical dispersion. In turn, these effects can produce a vertical emittance with a magnitude approaching that of the horizontal emittance.

To store the beam for long periods in an electron storage ring, it is necessary to avoid various types of beam loss mechanisms. These mechanisms may be categorized as singleparticle effects (which do not depend on the number of circulating electrons) and collective effects (which depend on the magnitude of the circulating beam current).

The most important single-particle loss mechanisms are the linear and non-linear resonances of the storage ring. These resonances can lead to transverse beam growth and resultant beam loss; thus, they must be avoided through the careful selection of storage-ring parameters and the accurate construction of storage-ring elements. A related problem is the correction of the natural chromaticities of the storage ring (defined as $\mathrm{p} d \nu / \mathrm{dp}$ ) through the use of carefully placed sextupoles. Gas scattering also leads to particle losses and is controlled by maintaining an extremely good vacuum throughout the storage-ring vacuum chamber.

Collective effects may be subdivided into incoherent and coherent mechanisms. An example of an incoherent effect is the space-charge defocusing of the electron beam, including image effects. This defocusing is normally unimportant for tightly bunched beams, because of the cancellation of electric and magnetic forces at relativistic energies. However, if ions become trapped by the potential well of the beam, these forces do not cancel completely, and the electron beam can be focused. Since compensation for this effect may be difficult, the ions must be cleared from the vacuum chamber. Another important incoherent effect is the Coulomb scattering of particles within the beam. In the Touschek effect, the transverse momentum of particles within a dense bunch is transformed by hard collisions into longitudinal momentum, and particles are lost from the longitudinal potential well of the bunch. The severity of this problem depends on the charge density within each bunch, so it is exacerbated by a storage-ring design that has a very small electronbeam emittance. Designing for an acceptably long Touschek lifetime for the electron beam requires a careful selection of storage-ring parameters. Multiple collisions within a bunch are another manifestation of Coulomb scattering, and they can lead to an increase in the electron-beam emittance. This effect is small as long as the growth time resulting from multiple collisions is long compared to the radiation damping time.

Transverse and longitudinal coherent instabilities, which are due to interactions between the beam and its environment, are encountered in electron storage rings, and they must be controlled to achieve a large circulating current of electrons. These instabilities can be roughly divided into coupled-bunch oscillations and single-bunch (or internal) oscillations.

The source of coupled-bunch instabilities is easy to understand. Since each beam bunch oscillates in a three-dimensional potential well with three characteristic frequencies, the many bunches represent many similar oscillators that are coupled through the beam environment and that can drive each other on-resonance. The primary method for reducing coherent effects is to provide a spread in the oscillator frequencies. Longitudinally, this may be accomplished by adding a "Landau Cavity" to make the potential well more non- 
linear. Transversely, an if quadrupole can be used to split the frequencies of the bunches. Alternatively, these instabilities may be controlled by altering the beam environment to decrease the coupling between bunches (reducing the impedance of the storage ring and rf cavities) or by adding active feedback to stabilize the bunches.

Internal-bunch modes are more difficult to correct. To control the transverse modes, the natural negative chromaticity of the storage ring must be corrected to zero by using sextupoles. For internal-bunch modes as well as coupled-bunch modes, the primary method of alleviating coherent instabilities is to reduce the impedance of the storage ring at high frequencies by providing a smooth vacuum chamber.

\section{Storage Ring}

The electron storage ring for the ALS has a nominal operating energy of $1.3 \mathrm{GeV}$, which has been chosen to provide intense photon beams of the desired energies.

\section{Design Considerations}

The design of the Advanced Light Source has been optimized to achieve two major goals. This facility is designed to provide intense photon beams (especially in terms of spectral brilliance) in the nominal energy range $0.1 \mathrm{eV}$ to $5000 \mathrm{eV}$, and the ALS is designed to provide very short pulses of synchrotron light (tens of picoseconds) for the many experiments with timing requirements in this range. In order to achieve these goals, the storage ring incorporates twelve long straight sections in which special magnetic devices, wiggler and undulators, will provide intense beams of synchrotron light. Wiggler and undulator magnets have become increasingly important and successful as high-intensity synchrotronradiation sources, and the capability to design and construct such devices has improved greatly. Therefore, the ALS will take advantage of the photon-beam intensity and the flexibility of such devices, and most or all of its photon beams will originate from wigglers and undulators rather than from bending magnets. Since wigglers can each service several photon beam lines, over 30 beam lines can eventually be constructed at the ALS from these sources as well as additional beam lines from bending magnets.

Aside from providing high photon-beam intensity, the use of wigglers and undulators has another important advantage. If the wiggler or undulator fields are higher than the bending-magnet field, the photons emitted will have higher energies than those from bending magnets. That is, a relatively low-energy electron beam can provide high-energy photons from a high-field wiggler or undulator. An electron energy of $1.3 \mathrm{GeV}$ appears optimal for the ALS, since it will provide intense photon beams with energies from 0.1 to $5000 \mathrm{eV}$, the major region of interest. This electron energy is high enough for a long beam lifetime, yet low enough to permit a very low electron-beam emittance.

For the benefit of synchrotron-radiation experiments requiring high-energy photons, the ALS will be operated at electron energies up to $1.9 \mathrm{GeV}$. Photons with energies up to $10 \mathrm{keV}$ will then be available from undulators, and photons with energies up to $40 \mathrm{keV}$ will be available from a superconducting wiggler. However, at higher electron-beam energies the ALS will have an increased electron beam emittance and, therefore, reduced spectral brilliance in some photon beams. Also, the electron bunch lengths will be increased.

The low electron-beam horizontal emittance is very desirable for a synchrotronradiation source, because a low emittance means a small cross-sectional area for the 
electron beam. In turn, a small cross-sectional area implies a high photon-beam spectral brilliance, as is evident from the definition of spectral brilliance given earlier. Furthermore, a small emittance for the electron beam permits the construction of very-highperformance undulators.

Several considerations are important in the choice of a frequency for the radiofrequency accelerating system. Foremost is the desire to provide a very short bunch length for the benefit of experiments with critical timing requirements. A fairly high frequency (around $500 \mathrm{MHz}$ ) has been selected to provide photon pulses approximately $35 \mathrm{ps}$ long. For those experiments requiring long dead times between successive pulses, the storage ring will be operated with a few electron bunches, at lower average electron currents.

\section{Major Parameters}

The major parameters of the ALS can best be understood by making a comparison (see Table 1) with existing electron storage rings dedicated to synchrotron radiation. The most prominent feature of the ALS design is the large number of straight sections for wigglers and undulators. These special synchrotron-radiation sources will provide greatly enhanced photon-beam performance, as compared with bending magnets, and the required straight sections account for the relatively large circumference of the ALS. The second noteworthy feature of the ALS is the very small design value of the horizontal emittance of the electron beam. This emittance has been carefully minimized in the ALS design in order to maximize the intensities of the photon beams, in general, and to increase greatly the spectral brilliance of the undulator photon beams, in particular. The excellent performance of wigglers and undulators permits the selection of modest values for the electron energy and electron current, while maintaining outstanding photon-beam performance. In the table, comparisons are given for Aladdin, the storage ring located near Madison, Wisconsin, and for the National Synchrotron Light Source (NSLS), which operates two storage rings located at the Brookhaven National Laboratory in New York. The Stanford Synchrotron Radiation Laboratory (SSRL) uses the SPEAR storage ring at Stanford, California.

Table 1

Electron Storage-Ring Design Parameters

\begin{tabular}{lccrcc}
\hline & $\begin{array}{c}\text { ALS } \\
\text { (Proposed) }\end{array}$ & Aladdin & $\begin{array}{c}\text { NSLS } \\
\text { (VUV) }\end{array}$ & $\begin{array}{c}\text { NSLS } \\
\text { (X-ray) }\end{array}$ & $\begin{array}{c}\text { SSRL } \\
\text { (SPEAR) }\end{array}$ \\
\hline Electron Energy (GeV) & 1.3 & 1 & 0.7 & 2.5 & 4 \\
& $(1.9$ max) & & & & \\
Electron Current (mA) & 400 & 500 & 1000 & 500 & 100 \\
$\begin{array}{l}\text { Circumference (m) } \\
\text { Horiz. Emittance }\end{array}$ & 182.4 & 88 & 51 & 170 & 234 \\
$\begin{array}{l}\left(10^{-8} \pi \text { m-rad) }\right. \\
\text { Bunch Length (ps) }\end{array}$ & 0.68 & 6 & 8 & 8 & 40 \\
& 23 & 80 & 400 & 400 & 100 \\
\hline
\end{tabular}


Table 2 presents a more detailed list of parameters for the ALS electron storage ring. It should be emphasized that the selection of parameters is a process of compromising among competing, and often conflicting, design considerations. For example, a low electron-beam emittance requires very strong focusing in the storage ring, but this focusing leads to large values of natural chromaticity, which can be difficult to correct. A more complex compromise arises from a consideration of the electron-beam lifetime, as limited by the intra-beam scattering (Touschek effect) and gas scattering. Since the Touschek effect depends on the electron energy, the electron current, the horizontal beam emittance, and the rf frequency and voltage, the choice of a set of these parameters is constrained by the necessity to design for an acceptable beam lifetime of more than 6 hours. Chromaticity is defined as $\mathrm{p} \mathrm{d} \nu / \mathrm{dp}$.

Table 2

Advanced Light Source Design Parameters

(Electron Storage Ring)

\begin{tabular}{lc}
\hline Electron Energy (GeV) & $1.3(1.9$ max) \\
Electron Current (mA) & 400 \\
Circumference (m) & 182.4 \\
Horiz. Emittance ( $\pi$ m-rad) & $6.8 \times 10^{-9}$ \\
No. of Superperiods & 12 \\
No. of Long Straight Sections & 12 \\
Length of Long Straight Sections (m) & 6 \\
Max. Horiz. Beta (m) & 13.3 \\
Max. Vert. Beta (m) & 13.3 \\
Horiz. Tune & 13.8 \\
Vert. Tune & 7.8 \\
Horiz. Chromaticity & -32.0 \\
Vert. Chromaticity & -17.5 \\
Energy Loss per Turn - Dipoles Only (keV) & 64 \\
Radiofrequency (MHz) & 499.65 \\
Harmonic Number & 304 \\
& \\
Dipole Field (T) & $1.9-$ GeV Operation: \\
Max. Quad. Gradient (T/m) & 22.9 \\
Energy Loss per Turn-Dipoles Only (keV) & 291 \\
\hline
\end{tabular}

Figures 3 and 4 show the spectral brilliance of the ALS photon beams for an electron current of $400 \mathrm{~mA}$. The spectral output of undulators consists of a number of sharp peaks, as shown in Figure 2. Figure 3 shows smooth curves drawn through the maxima of the spectral peaks for each undulator. The top curve in Figure 4, however, is the envelope of the curves in the preceding graph. For all ALS and NSLS curves in Figure 4, the electron beam vertical emittance was assumed to be one-tenth the horizontal emittance. Since the undulator spectra consist of peaks, it is necessary to vary the undulator fields to scan over 


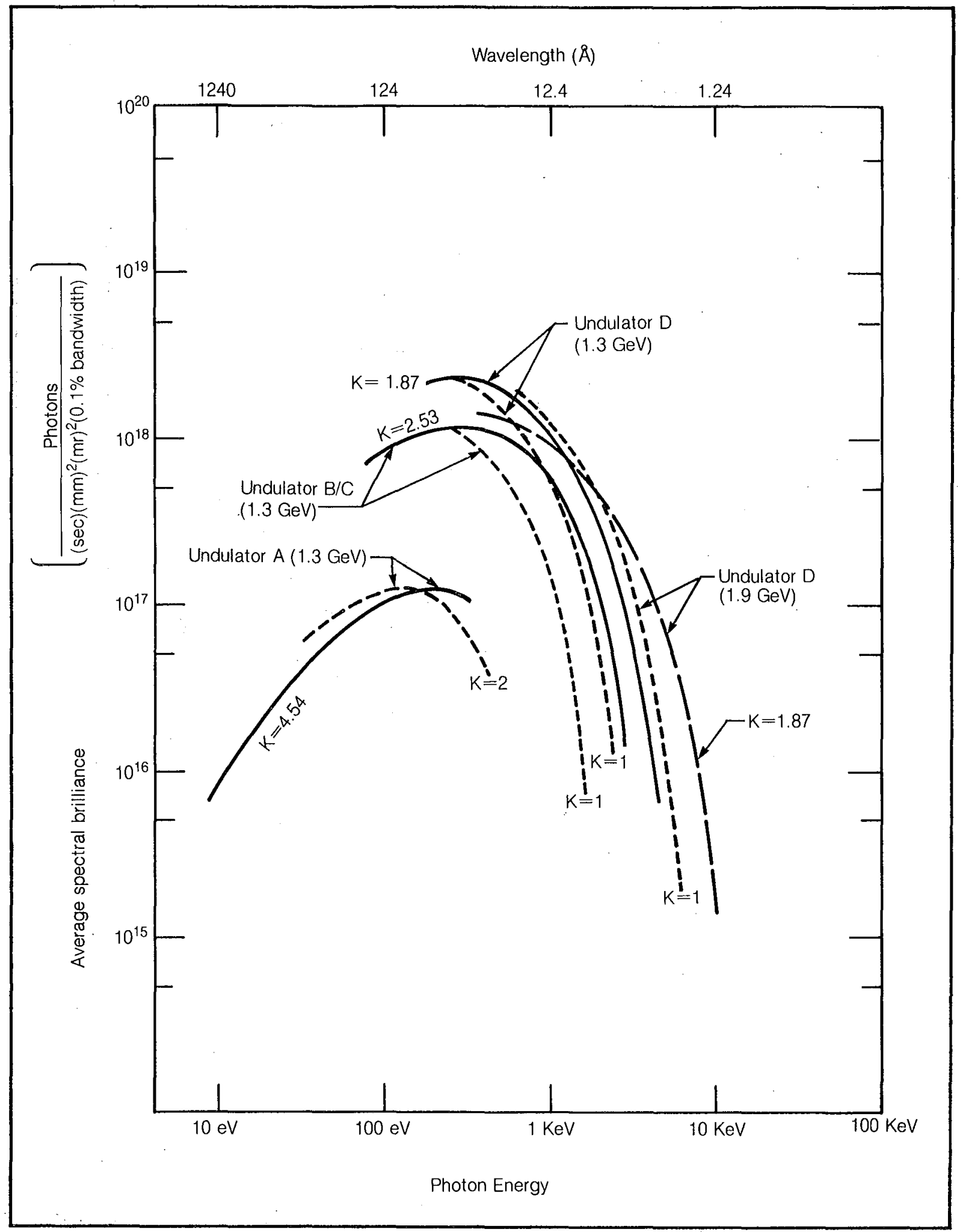

Figure 3. Spectral brilliance of ALS undulators.

XBL 833-8927 


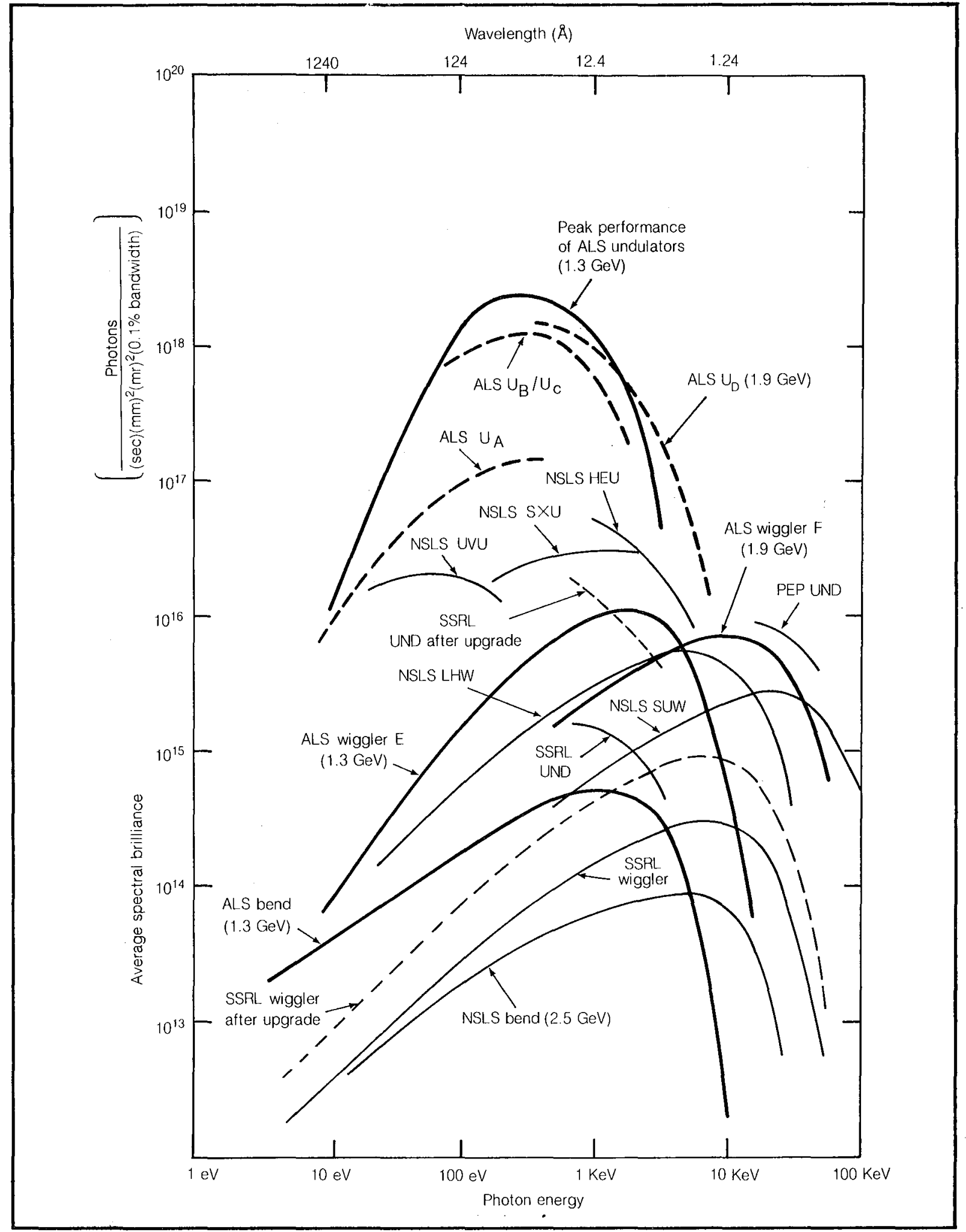

Figure 4. Spectral brilliance comparisons.

XBL 833-8928 
all desired photon energies. For undulators constructed with permanent-magnet technology, the field strengths are varied by changing the magnet gaps. The label "average spectral brilliance" means that the spectral brilliance has been averaged over time, i.e., the small duty factor of the electron beam has been ignored. While an electron bunch traverses an undulator, for example, the instantaneous spectral brilliance is much higher than is shown in these figures. The instantaneous electron current is tabulated for several operating modes later in this section.

To compute the spectral brilliance introduced in the above, one starts from the following quantity

$$
\text { spectral brightness }=\frac{\text { number of photons }}{(\mathrm{s})(\mathrm{mr})^{2}(0.1 \% \text { bandwidth })}
$$

This quantity is unambiguously determined from electromagnetic theory. The spectral brilliance is then obtained by dividing the spectral brightness by an effective source area $S$, which is given by

$$
\mathrm{S}=2 \pi \Sigma_{\mathrm{x}} \Sigma_{\mathrm{y}}, \quad \Sigma_{\mathrm{x}, \mathrm{y}}=\sqrt{\sigma_{\mathrm{x}, \mathrm{y}}^{2}+\left(\lambda \mathrm{L}+\sigma_{\mathrm{x}, \mathrm{y}}^{2} \mathrm{~L}^{2}\right) / 4}
$$

in the case of a wiggler or an undulator of length $\mathrm{L}$. Here, $\sigma_{\mathrm{x}, \mathrm{y}}$ and $\sigma_{\mathrm{x}, \mathrm{y}}^{\prime}$ are the onestandard-deviation sizes and angular divergences of the electron beam, and $\lambda$ is the wave length of the observed photon. $\mathrm{S}$ is a measure of the effective source area including the effects of diffraction and the extended nature of the source (i.e., depth of field effects). However, it should be mentioned that the definition of $S$ is somewhat arbitrary and different authors may use different numerical factors. For a bending magnet source, both the diffraction effect and the depth of the field effect can be neglected (except in the far infrared region), so that

$$
\mathrm{S}=2 \pi \sigma_{\mathrm{x}} \sigma_{\mathrm{y}}
$$

Figure 5 shows the photon flux from the bending magnets at the ALS and the much higher photon fluxes from the ALS wigglers. Note that a superconducting wiggler magnet can provide photons with energies up to about $40 \mathrm{keV}$. These curves are calculated for a current of $400 \mathrm{~mA}$.

The theory of electron storage rings has not been developed to the point where we can predict with complete certainty the peak operating parameters that can be achieved with a storage ring. Nevertheless, in this section we shall present the approximate parameters for a few ALS standard operating modes and also discuss the limitations on these parameters. The "initial performance goals" which are given in Table 3 represent the state-of-the-art performance as scaled from existing storage rings, and these figures may be used as a general guide by prospective users of the ALS. The peak currents in this table can be used to calculate instantaneous spectral brilliances and photon fluxes from Figures 4 and 5, which show curves for $400 \mathrm{~mA}$ average current. The "ultimate performance goals" represent some extrapolation from current experience, and they should not be used to plan synchrotron radiation experiments at this time. However, these ultimate goals are consistent with presently understood limits on storage ring performance, and it is our intent to design the 


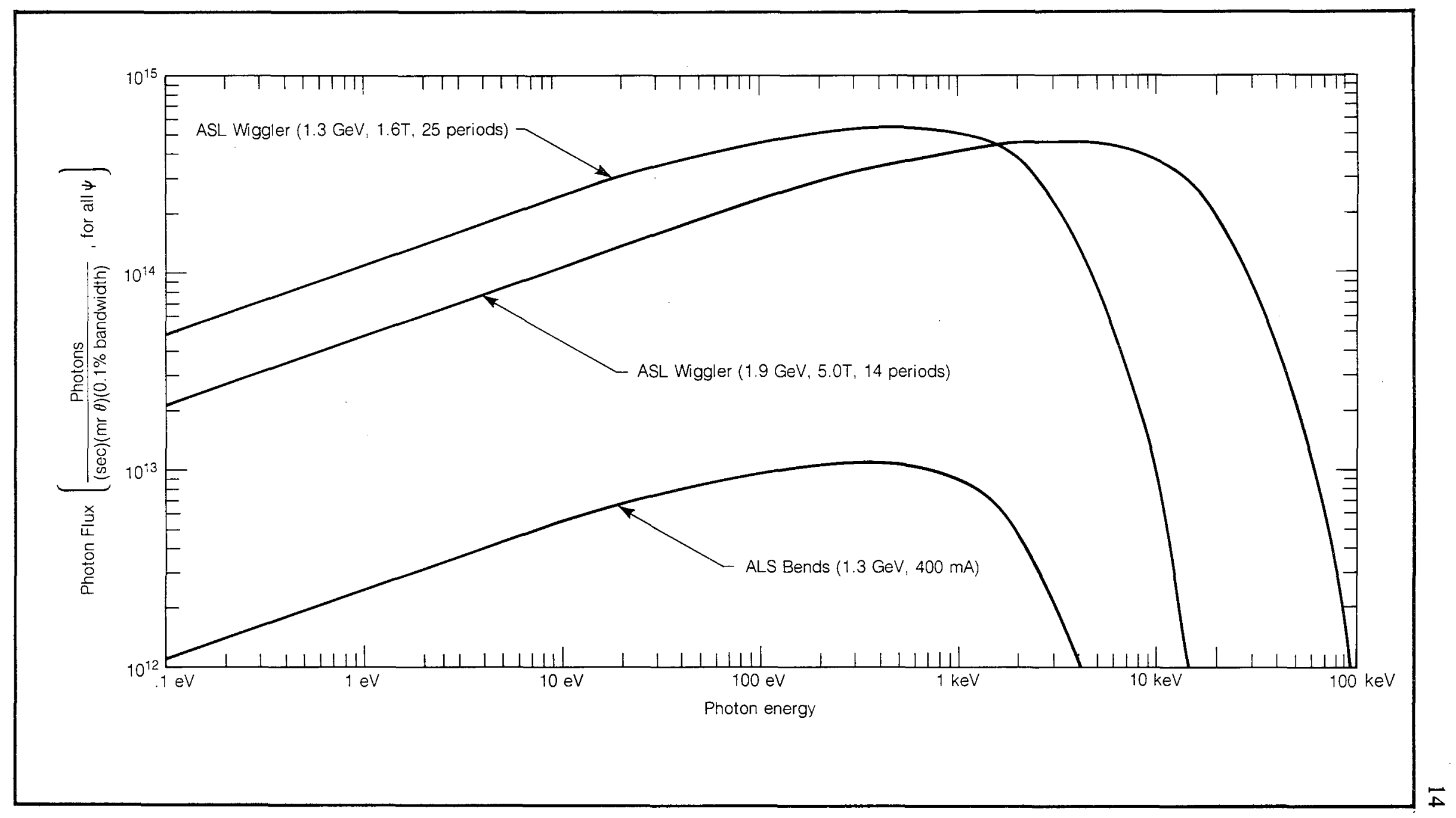

Figure 5. Photon flux. 
ALS so that it can be operated at these conditions.

Table 3

Approximate Parameters for

ALS Standard Operating Modes

\begin{tabular}{lrrrrrr}
\hline Energy (GeV) & 0.9 & 1.3 & 1.3 & 1.3 & 1.3 & 1.9 \\
No. of Bunches & 250 & 250 & 250 & 20 & 1 & 250 \\
Initial Performance Goals & & & & & & \\
$\quad$ Average Current (mA) & 400 & & 400 & 200 & 7.5 & 400 \\
$\quad$ Bunch Length (ps) & 23 & & 23 & 38 & 35 & 27 \\
$\quad$ Peak Current (A) & 34 & & 34 & 128 & 104 & 29 \\
$\quad$ Touschek Lifetime (h) & 6 & & 16 & 5 & 6 & 85 \\
Ultimate Performance Goals & & & & & & \\
$\quad$ Average Current (mA) & 400 & 25 & 400 & 400 & 50 & 400 \\
Bunch Length (ps) & 23 & 8 & 23 & 50 & 90 & 27 \\
Peak Current (A) & 34 & 6 & 34 & 194 & 270 & 29 \\
\hline
\end{tabular}

In estimating the peak operating parameters presented in Table 3, a number of possible limitations to storage ring performance must be considered. These limitations are discussed in the rest of this section. The second column in Table 3 indicates that extraordinarily short pulse lengths may be achieved by specially tuning the ALS to provide a lower momentum compaction factor, although at the cost of increased beam emittance.

The threshold current for the longitudinal microwave instability [4] is given by the relation

$$
\mathrm{I}_{\mathrm{th}}=\frac{\mathrm{F} \nu_{\mathrm{s}}^{2}(\mathrm{E} / \mathrm{e})}{\sqrt{2 \pi} \alpha\left|\frac{\mathrm{Z}}{\mathrm{n}}\right|}\left(\frac{\sigma_{\mathrm{s}}}{\mathrm{R}}\right)^{3}
$$

where $\nu_{\mathrm{s}}$ is the synchrotron tune, $\sigma_{\mathrm{s}}$ the rms bunch length, $\mathrm{F}$ a form factor, and $\mathrm{Z}$ the broadband component of the longitudinal impedance for mode $\mathrm{n}$. When the current stored exceeds this value, the bunch lengthens to satisfy the condition. The maximum of $|\mathrm{Z} / \mathrm{n}|$ has been estimated to be $8 \Omega$ for SPEAR and less than $1 \Omega$ (excluding rf cavities) for the large rings PEP and PETRA, located respectively at Stanford in California and at DESY in Hamburg, Germany. For the ALS with smooth vacuum chamber construction, $2 \Omega$ or less should be realizable. At frequencies above cutoff, $|\mathrm{Z} / \mathrm{n}|$ decreases to the free-space impedance, which for the ALS beam pipe will introduce a few tenths ohm. The bunch lengths for various operating conditions are summarized in Table 3 , where the rolloff of $|\mathrm{Z} / \mathrm{n}|$ for short bunches is scaled from SPEAR until the free-space value above cutoff is attained.

Transverse single-bunch instabilities can be driven by the broadband transverse impedance. The slow-growth "head-tail" instability will be stabilized in the ALS by 
sextupole correction of the machine chromaticity. The fast-growth transverse instability sets an upper limit on the maximum bunch current that can be stored. For the ALS, the threshold current is over $75 \mathrm{~mA} /$ bunch and should not interfere with the planned modes of operation, as indicated in Table 3.

Narrowband impedances from, for example, parasitic modes of the rf cavities, can produce long wake fields that couple succeeding bunches and cause longitudinal instability. Care in the design of the rf system (to reduce the $\mathrm{Q}$ of higher modes of the cavity) is fundamental in eliminating this coherent effect. The rise time $\tau$ for the dipole mode is given by [5]

$$
\frac{1}{\tau}=\frac{\omega_{\mathrm{o}} \mathrm{el} \alpha}{4 \pi \nu_{\mathrm{s}} \mathrm{E}}\left[\mathrm{n} \mathrm{Z} \mathrm{Z}_{\mathrm{n}} \mathrm{e}^{-\frac{1}{2} \mathrm{n}^{2} \sigma_{\theta}^{2}}\right]
$$

and should be the most severe coupled-bunch stability. From past experience in damping cavities at the Photon Factory (KEK, Japan) and at the Synchrotron Radiation Source (Daresbury, England), it is estimated that at approximately $75 \mathrm{~mA}$, the growth rate for the fastest growing mode will equal the synchrotron radiation damping rate. It has been the experience at both the NSLS VUV ring and the KEK Photon Factory that higher currents do not lead to catastrophic beam loss, but rather to finite longitudinal bunch oscillations. For the KEK Photon Factory, which has a comparable instability threshold, the longitudinal coupled-bunch instability has not been a limiting factor in attaining more than $200 \mathrm{~mA}$ of stored beam. A small second rf system, which would provide either a bunch-to-bunch shift or an internal-bunch spread in synchrotron frequency, will provide sufficient damping to achieve the design current of $400 \mathrm{~mA}$ without any residual oscillation. It has also been found at SPEAR that certain configurations of filled buckets are more stable than others. The ALS injection scheme provides flexibility in selecting configurations of bunches, so that the most stable operating mode can be found. Transverse coupled-bunch modes will be less severe and can be stabilized by natural radiation damping or, if necessary, by a modest bunch-to-bunch tune spread from rf quadrupoles.

In addition to the single-particle synchrotron radiation, energy loss can occur through higher-order-mode excitation [6] and coherent synchrotron radiation [7] in the bending magnets. Conservative estimates indicate that higher-order-mode losses will dominate over coherent synchrotron radiation even for the shortest bunches in the ALS. For the 4-bunch, 30-mA mode, higher-order-mode losses of $80 \mathrm{kV} /$ turn can be expected. For the 20-bunch, 200-mA mode the losses will be $95 \mathrm{kV} /$ turn. Great care must be taken in the construction of storage ring components to avoid vacuum chamber components which can behave as high-frequency resonant cavities. The passage of dense bunches of electrons can excite intense standing waves in such cavities and produce component failure through overheating.

A primary goal in the design of the ALS is to provide electron bunches of extremely short duration with many milliamperes of current per bunch. A variety of effects which are sensitive to the planned high peak currents has led to the choice of an rf system which can provide $3 \mathrm{MeV}$ peak electron energy gain per turn. First of all, a high rf voltage provides longer Touschek lifetimes. Furthermore, providing $3 \mathrm{MeV}$ energy gain per turn results in a zero-current bunch length of $20 \mathrm{ps}$ at $1.3 \mathrm{GeV}$; thus we have a safety margin in achieving a 
35-ps bunch length, even if the storage ring impedance for smooth rings turns out to be inductive at high frequencies as is suggested by experience at PETRA. Finally, both the fast-growth transverse-instability threshold and the coupled-bunch longitudinal-instability threshold are eased by operation at higher synchrotron frequencies. These considerations have led to the selection of a powerful radiofrequency system for the ALS.

The Touschek lifetime decreases with decreasing beam dimensions and is therefore sensitive to the low transverse emittance and short bunch length goals for the ALS. However, the low transverse emittance design of the ALS also limits the transverse momentum available to scatter electrons outside the rf bucket. With an rf voltage of $3 \mathrm{MV} / \mathrm{turn}$, which will provide a $35 \mathrm{ps}$ bunch length at several milliamperes per bunch, the nominal bucket height is $\pm 5 \%$. However, the dynamic aperture sets more a severe limitation on the momentum deviation that can be stably stored. Orbit tracking studies indicate that momentum deviations in the approximate range of $2.4 \%$ to $3.7 \%$ (depending on the location of the scattering event in the ring) can be tolerated. With these limits the Touschek lifetime was calculated using the local lattice functions. Increasing the single-bunch current reduces the achievable Touschek lifetime, and some parameter optimization with respect to current, lifetime, emittance, bunch length and energy is needed in determining which modes of operation will be most satisfactory to users.

The dynamic aperture of a storage ring is the useful aperture of the vacuum chamber within which beam particles can execute stable oscillations. The dynamic aperture can be established by particle tracking studies in which the stability of particle trajectories can be tested for many thousands of turns around the storage ring. Such studies for the ALS have shown that, when a carefully optimized system of sextupoles is used to correct the natural chromaticities, the dynamic aperture is approximately equal to the physical aperture of the vacuum chamber.

Scattering from residual gas and intrabeam scattering can reduce stored-beam lifetime. At a pressure of $10^{-9}$ torr, the gas scattering lifetime with a $400-\mathrm{mA}$ beam current is estimated to be 13 hours, if the minimum vertical gap at an undulator is $9 \mathrm{~mm}$. Intrabeam scattering can also lead to both longitudinal and transverse emittance growth. For the ALS, the synchrotron damping rate exceeds the rates of growth by at least an order of magnitude, and thus there should be no significant impact.

Even at a vacuum of $10^{-9}$ torr, the electron beam can become neutralized in a few seconds, if positive ions become trapped in the potential well formed by the beam. These ions can produce a focusing force that is nonlinear with respect to displacement from the central orbit, so they can cause both tune shifts and tune spreads in the electron beam. Even worse, the electron-beam emittance can grow substantially (possibly through encountering resonances because of the tune shift), and the beam lifetime can be substantially reduced.

Although the presence of ions has been seen to improve beam behavior in some cases, it seems clear that ion trapping should be avoided in the ALS if necessary. There are three standard methods of avoiding ion trapping. First, the storage ring can be run with fewer bunches of electrons, so that a fraction of the circumference is empty. The rest of this section will discuss this strategy in some detail. Second, clearing electrodes may be used to remove the ions. However, the addition of clearing electrodes may be awkward in some locations and is likely to produce an unattractive increase in the impedance of the storage ring. Third, the electron beam can be replaced with a positron beam, at a substantially 
increased cost. This last option is discussed in some detail at the end of the Section titled "Injection System."

Ion trapping by bunched electron beams can be simulated using an analytic model in which the space-charge force of an electron bunch is represented by a thin focusing lens $[8,9]$. The electron bunch has a Gaussian density distribution, so the focusing force is not linear with displacement from the central orbit. However, for simplicity we assume a linear lens whose strength is taken to be the actual focusing force near the electron beam axis. Therefore, the electron beam is represented as a lattice of converging lenses in which ions execute transverse oscillations. For ion motion to be stable in such a lattice, the ion mass must exceed a critical value given by

$$
A_{c}(\text { a.m.u. })=2\left(\frac{\pi R}{n}\right)^{2} \frac{m_{e}}{m_{p}} \frac{I}{I_{o}} \frac{1}{\sigma_{y}\left(\sigma_{x}+\sigma_{y}\right)}
$$

where $\mathrm{R}=$ mean radius of storage ring

$\mathrm{n}=$ number of equally-spaced electron bunches

$I=$ total electron beam current

$I_{0}=$ Alfven current

$\sigma_{\mathrm{x}}, \sigma_{\mathrm{y}}=$ transverse beam dimensions.

Ions with masses lower than $A_{c}$ will be overfocused vertically and therefore not be trapped.

The above expression can usefully be re-written as

$$
A_{c}(\text { a.m.u. })=K \frac{I}{n^{2}}
$$

where the constant $\mathrm{K}$ is a figure of merit that depends solely on the parameters of the storage ring. A large value of $\mathrm{K}$ is preferred, since it indicates a relative immunity from ion trapping.

Table 4 shows how various storage rings compare, in terms of ion trapping. First of all, it is evident that the ALS has unusually large values of $\mathrm{K}$, meaning that the ALS is relatively immune to ion trapping because of its large radius and small beam size (which in turn is due to a low emittance). Second, the operating modes of the ALS, other than the 250-bunch case, all have $A_{c}$ larger than 10. Since the NSLS VUV ring does not exhibit ion trapping with a value of $A_{c}$ equal to 10.45 (for single-bunch operation), it seems that ion trapping is not likely at the ALS when the number of bunches is no more than about 20 .

It is believed that carbon monoxide is a major component of the gas which is found within operating electron storage rings. Therefore, it is reasonable to assume that singlycharged, molecular carbon monoxide ions (mass 28) might present a serious problem in terms of ion trapping. Since the above model predicts that ions above mass 10 could be trapped at the VUV ring during single-bunch operation, and since ion trapping was not observed, it appears that this model is somewhat pessimistic. 
Table 4

Ion Trapping in Storage Rings

\begin{tabular}{lcccrlll}
\hline Name & $\mathrm{E}(\mathrm{GeV})$ & $\sigma_{\mathrm{x}}, \sigma_{\mathrm{y}}(\mathrm{mm})$ & $\mathrm{K}$ & $\mathrm{n}$ & $\mathrm{I}(\mathrm{A})$ & \multicolumn{1}{c}{$\mathrm{A}_{\mathrm{c}}$} & Ions trapped \\
\hline ADONE & 1.5 & $3.5,2.0$ & 14.42 & 3 & 0.6 & .96 & yes \\
DORIS & 3.0 & $6.0,0.3$ & 644 & 480 & 0.9 & .0025 & yes \\
NSLS VUV & 0.7 & $0.66,0.21$ & 209 & 9 & 0.10 & 0.26 & yes \\
& 0.7 & $0.66,0.21$ & 209 & 1 & 0.05 & 10.45 & no \\
SPEAR & 2.5 & $3.0,0.55$ & 411 & 4 & 0.1 & 2.57 & probably \\
ALS & 0.9 & $0.114,0.036$ & 90,400 & 20 & 0.2 & 45.2 & \\
& 0.9 & $0.114,0.036$ & 90,400 & 250 & 0.4 & 0.58 & \\
& 1.3 & $0.165,0.052$ & 43,200 & 20 & 0.2 & 21.6 & \\
& 1.3 & $0.165,0.052$ & 43,200 & 250 & 0.4 & 0.28 & \\
& 1.3 & $0.165,0.052$ & 43,200 & 4 & 0.03 & 81.0 & \\
& 1.3 & $0.165,0.052$ & 43,200 & 4 & 0.2 & 540 & \\
& 1.9 & $0.241,0.076$ & 20,300 & 20 & 0.2 & 10.2 & \\
& 1.9 & $0.241,0.076$ & 20,300 & 250 & 0.4 & 0.13 & \\
\hline
\end{tabular}

A series of numerical simulations was carried out to check the analytic model. Ions were tracked through a series of lenses either with linear forces or with non-linear forces (Gaussian electron bunches). The simulation results confirm the analytic calculations. In particular, these simulations do not reveal any ion trapping which is not predicted by the analytic calculation, and ions are usually lost to the wall in less than 100 microseconds. Consequently, ion accumulation appears unlikely in the ALS, except in the many-bunch operating modes.

Should ion trapping occur in the many-bunch mode, linear and non-linear numerical simulations indicate it may be possible to remove ions by leaving a single large gap in the train of electron bunches. For example, one could have 250 consecutive electron bunches followed by 54 empty if buckets. The ions are propelled outward by the series of converging lenses, and they are lost as the gap passes their azimuthal position. This strategy has been used successfully at DORIS.

\section{ALS Lattice}

The lattice of the ALS electron storage ring is designed to accommodate a large number of radiation-source insertion devices, to produce a very low electron beam emittance, to have favorable values of the various lattice functions at the long straight sections, to have an acceptable natural chromaticity, to have a high periodicity to reduce structure resonances, and to have low beta functions to minimize beam loss due to gas scattering.

The ring is divided into twelve superperiods, and it contains twelve 6-meter straight sections for undulators, for wigglers, and for injection. The beta functions and the dispersion function are shown in Figure 6. The dispersion is zero in the long straight sections to minimize source widths and angular divergences. Furthermore, because the wigglers and 
undulators are located where the dispersion is zero, they will actually tend to reduce the electron-beam emittance. Figure 7 shows the overall layout of the ALS storage ring and the many long straight sections, which are the dominant feature of this lattice design. Figure 8 shows a more detailed view of the major magnets in a portion of this ring.

The unusually low emittance of the ALS storage ring is achieved by arranging to have low values of momentum dispersion and horizontal beta function in the bending magnets. Because the behavior of this dispersion is controlled solely by the single quadrupole, QF, located between each pair of dipoles, the tune and beta values may be controlled independently (within limits) by the quadrupole triplets located near the long straight sections. Studies have shown that this lattice can readily be retuned to compensate for the substantial vertical edge focusing of a superconducting wiggler.

The design of the ALS lattice leads to reasonable values of the natural chromaticity and also provides appropriate locations to place sextupole magnets for correcting this chromaticity.

The required vacuum-chamber aperture in the ALS, sometimes called the "beam stay clear," has been set at $\pm 32 \mathrm{~mm}$ horizontally and $\pm 24 \mathrm{~mm}$ vertically. These parameters were selected after considering the betatron oscillations of electrons, the momentum dispersion in the storage ring, the aperture requirements for injection, and an allowance for closed-orbit errors.

Particle-tracking studies using the computer program PATRICIA [10] have been conducted to study the useful aperture of the ALS, which is defined as the maximum amplitude of stable transverse oscillations. Results show that the useful aperture is about twice that required for beam injection into the storage ring and about 100 times the root-meansquare amplitude of the damped beam; the useful aperture is also roughly equal to the physical aperture given above.

Magnet construction errors, magnet setting errors, and closed-orbit errors can all produce a growth in the electron-beam emittance. A preliminary numerical simulation of these effects has been completed, and the case considered was one in which closed-orbit errors $( \pm 3 \mathrm{~mm}$ horizontally and $\pm 1 \mathrm{~mm}$ vertically) were produced by random transverse offsets of quadrupoles and in which the sextupoles were set to correct the chromaticities to zero. No significant emittance growth was found. We plan to perform more detailed simulations in which the effects of closed-orbit corrections, magnet errors, and wigglers are all taken into account.

\section{Ring Magnets}

The magnets for the ALS electron storage ring present no unusual engineering difficulties. Although the accurate construction and alignment of storage-ring components will be crucial to the proper operation of the ALS, existing storage-ring technology (described below) is equal to this challenge. The ring magnets consist of four basic types: dipole magnets to bend the beam, quadrupole magnets to focus the beam, sextupole magnets for chromaticity corrections, and small dipole magnets for beam steering.

The storage-ring bending magnets are "C" magnets (see Figure 9) to permit the convenient extraction of the synchrotron radiation. Since the synchrotron-radiation beam and the electron beam leave a straight section along the same path, they separate only at the next bending magnet, where the beam ports for the photon beam lines are located. The magnets are straight, and the sagitta required for the curvature of the electron beam 


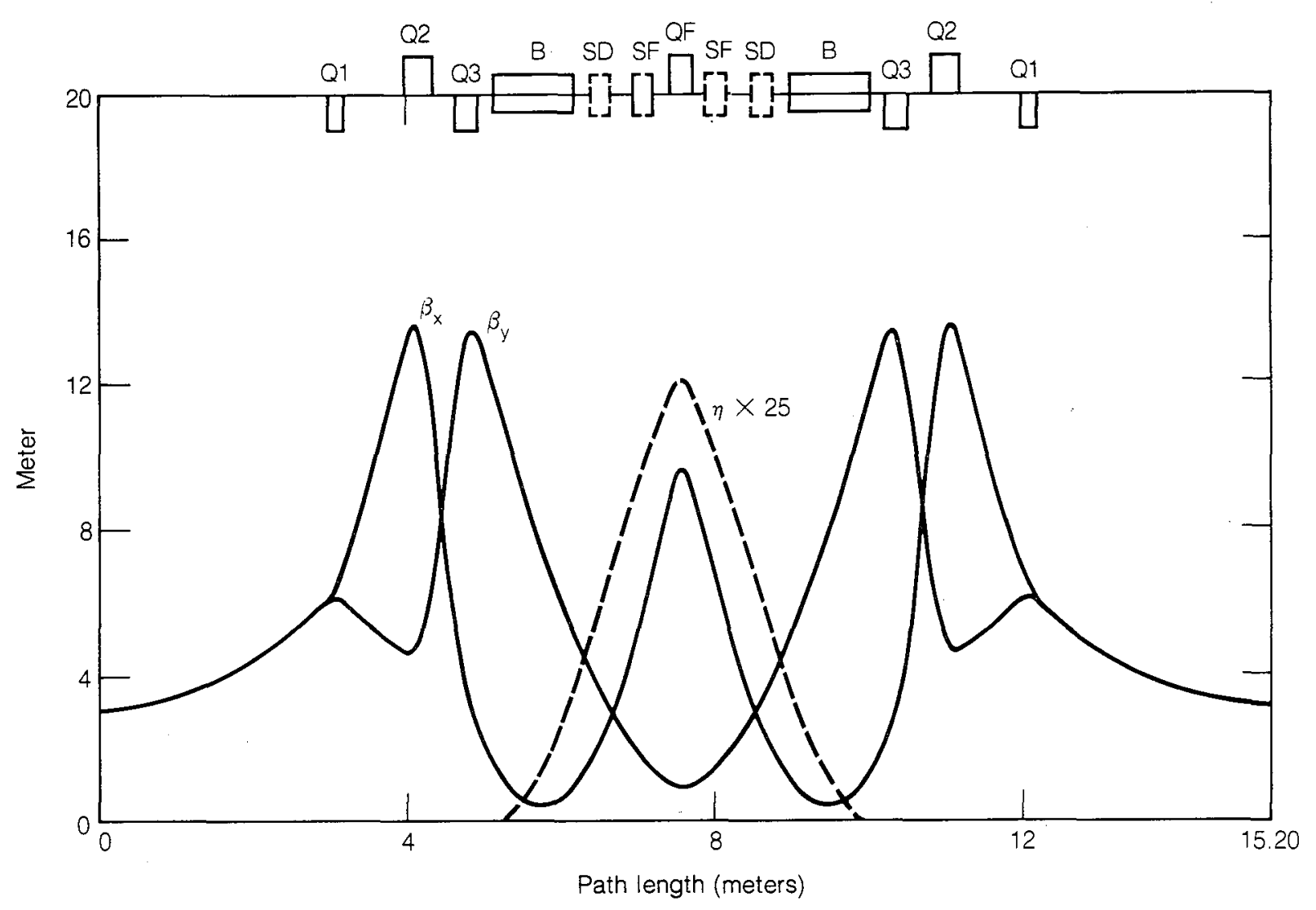

Figure 6. Storage Ring Lattice functions. 


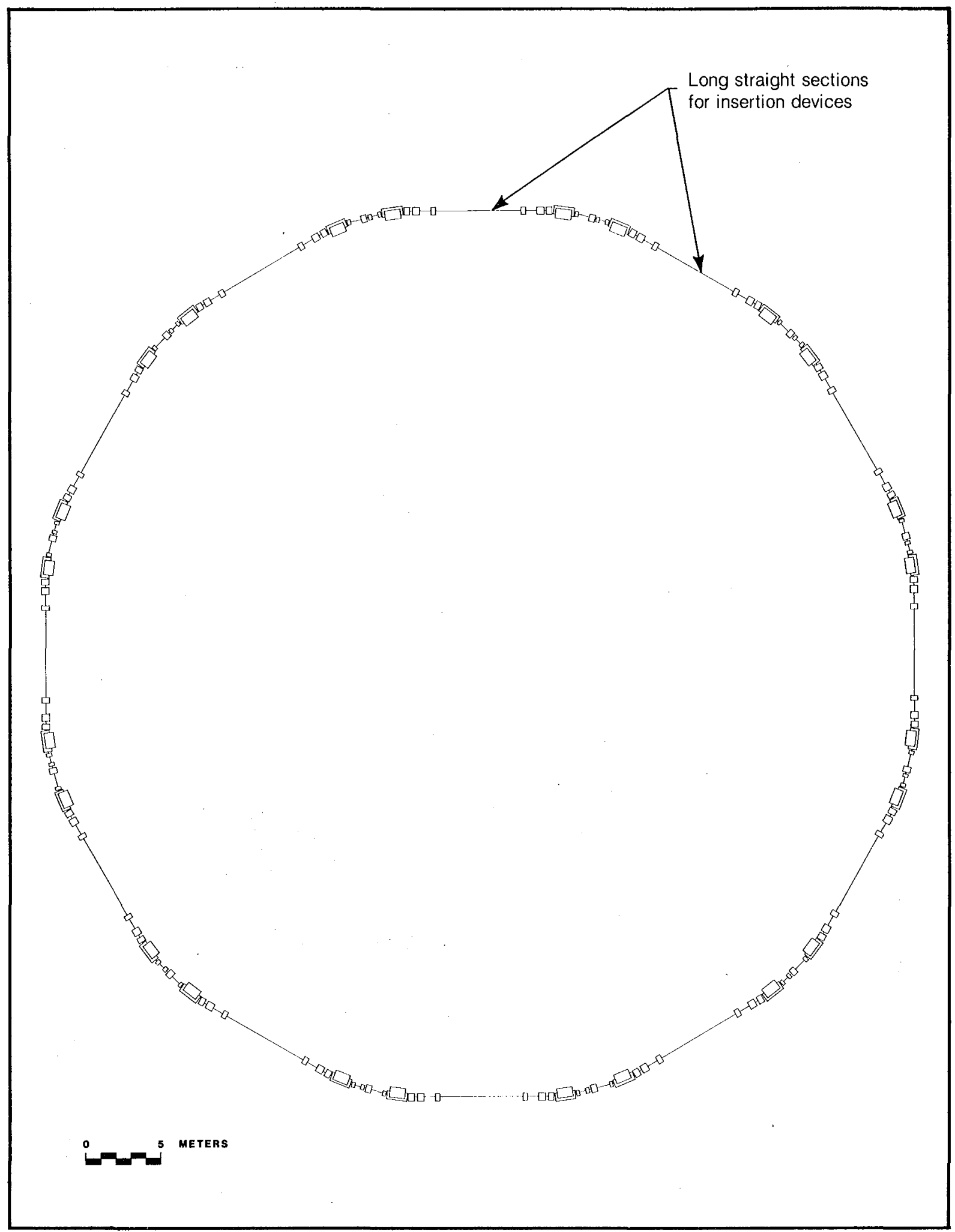

Figure 7. Storage ring.

XBL 833-8930 


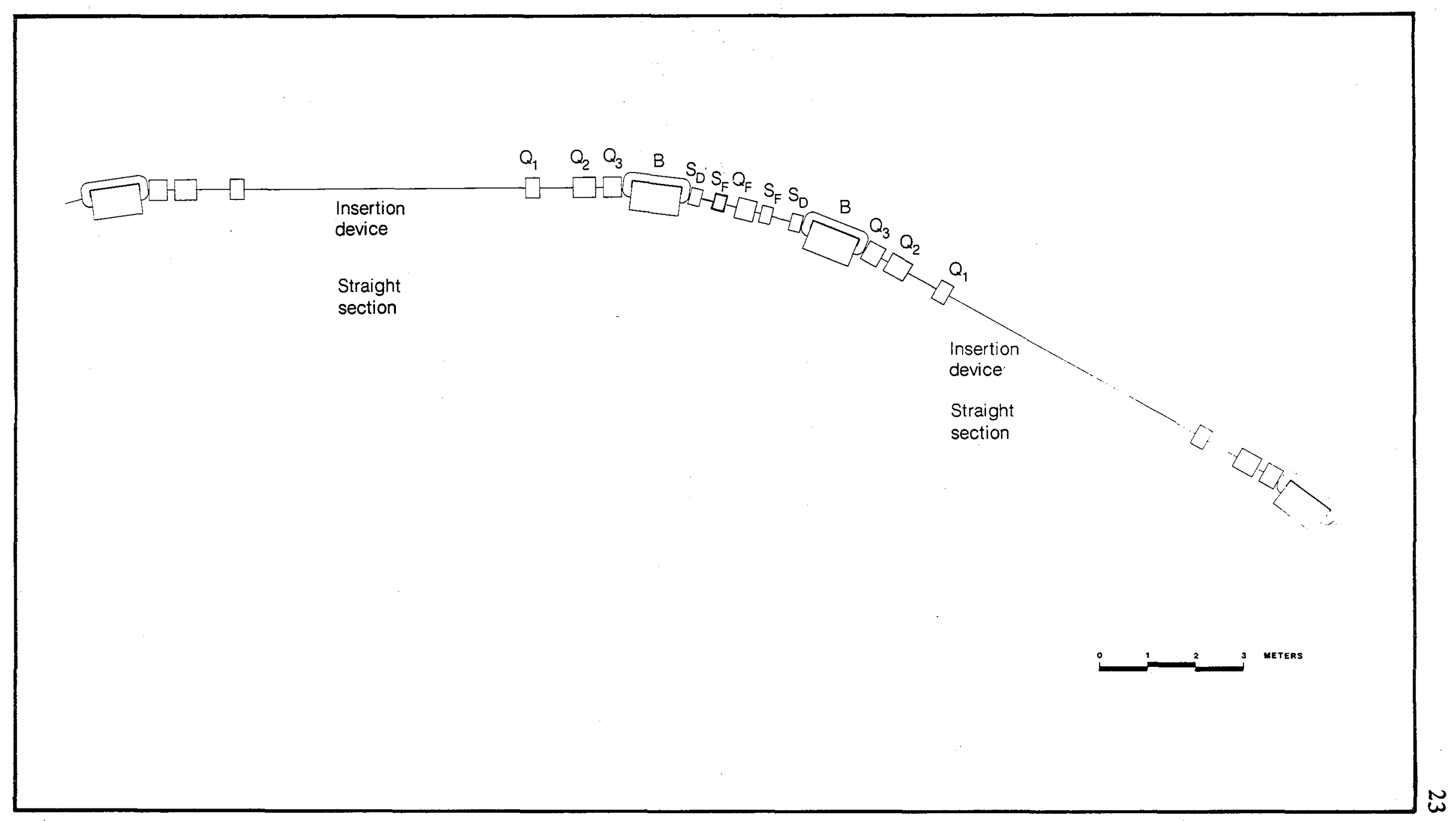

Figure 8. Detail of storage ring. 
trajectory is accommodated by a large pole area. These bending magnets are laminated, and the entire " $\mathrm{C}$ " shape is punched in one piece to ensure structural rigidity and to maintain the required high precision poletip contour. Table 5 is a short list of storage-ring bending-magnet parameters for $1.9-\mathrm{GeV}$ operation.

Table 5

Storage Ring Dipole-Magnet Parameters

\begin{tabular}{lc}
\hline Number required & 24 \\
Bending angle (degrees) & 15 \\
Field, 1.9 GeV (T) & 1.6 \\
Gap (cm) & 6.5 \\
Magnetic length (m) & 1.04 \\
Current (A) & 958 \\
Voltage, per magnet (V) & 22.1 \\
Power, per magnet, $1.9 \mathrm{GeV}(\mathrm{kW})$ & 21.2 \\
\hline
\end{tabular}

There are a large number of quadrupole magnets in the ALS electron storage ring because of the need to provide very strong focusing. These quadrupoles may be divided into four groups by magnet lengths: $20,37,29$, and $30.5 \mathrm{~cm}$. The aperture diameter is $6.5 \mathrm{~cm}$ for all magnets, as shown in Figure 10. In the ALS design, the synchrotron radiation from a wiggler or undulator passes through three quadrupole magnets before it leaves the ring at a bending magnet. Therefore, the quadrupole magnets must be designed to accept a vacuum chamber that is horizontally enlarged to transmit a wide, flat "fan" of synchrotron radiation. Some key parameters of the quadrupole magnets at $1.9 \mathrm{GeV}$ are given in Table 6.

Table 6

Ring Quadrupole-Magnet Parameters

\begin{tabular}{lcccc}
\hline Quadrupole Type & Q1 & Q2 & Q3 & QF \\
Number Required & 24 & 24 & 24 & 12 \\
Aperture Diameter (cm) & 6.5 & 6.5 & 6.5 & 6.5 \\
Effective Length (cm) & 20.0 & 37.2 & 29.1 & 30.5 \\
Maximum Gradient, 1.9 GeV (T/m) & 10.7 & 22.8 & 22.8 & 22.8 \\
Current (A) & 280 & 630 & 630 & 630 \\
Voltage, per magnet (V) & 1.8 & 7.0 & 5.8 & 6.0 \\
Power, per magnet, 1.9 GeV (kW) & 0.5 & 4.4 & 3.7 & 3.8 \\
Total Power for Circuit (kW) & 12 & 106 & 88 & 46 \\
\hline
\end{tabular}

Forty-eight sextupole magnets are used to correct the natural chromaticity of the storage ring. These magnets also have a $6.5-\mathrm{cm}$-diameter aperture (see Figure 11) and are 


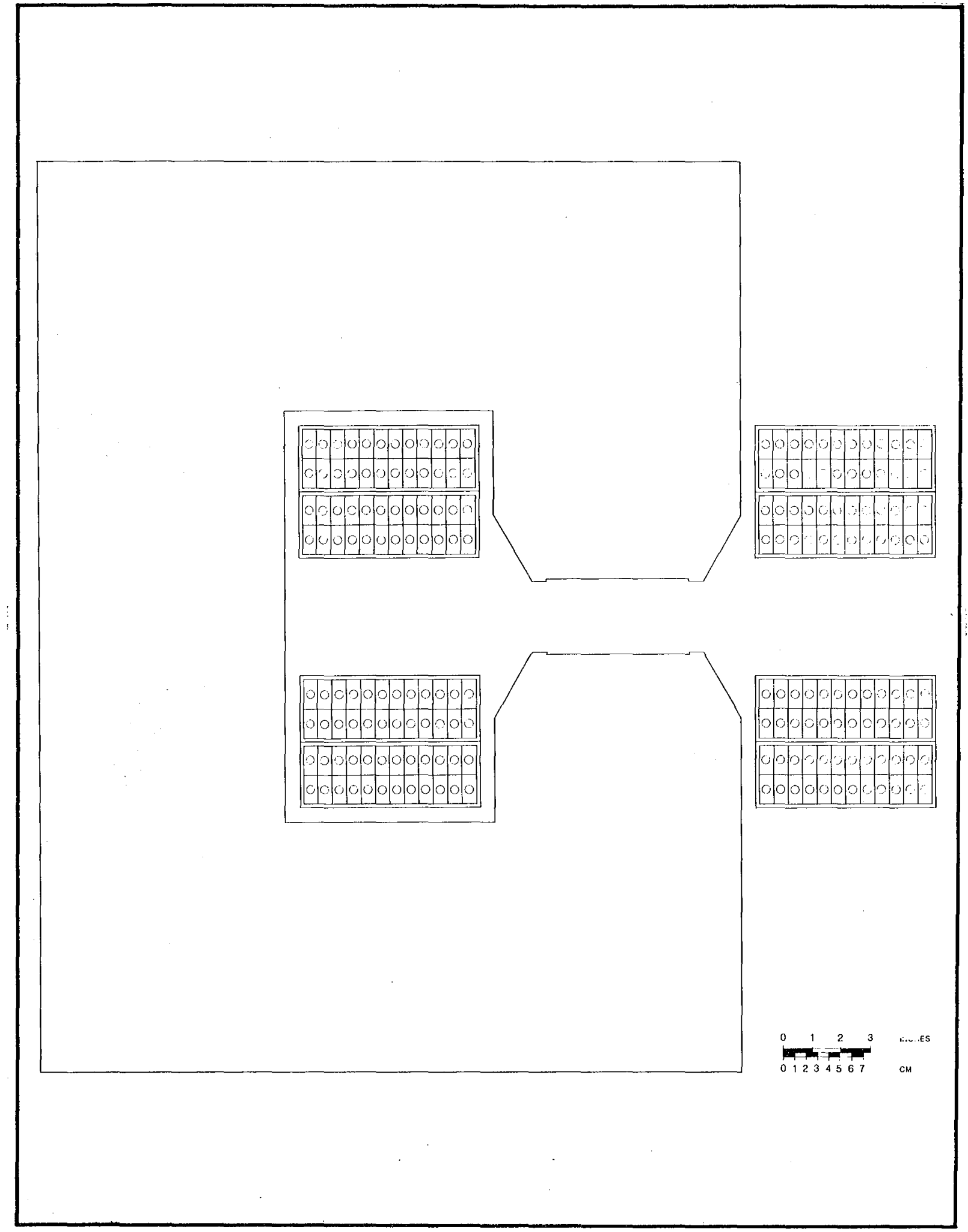

Figure 9. Storage ring bending magnet cross section.

XBL 833-8932 


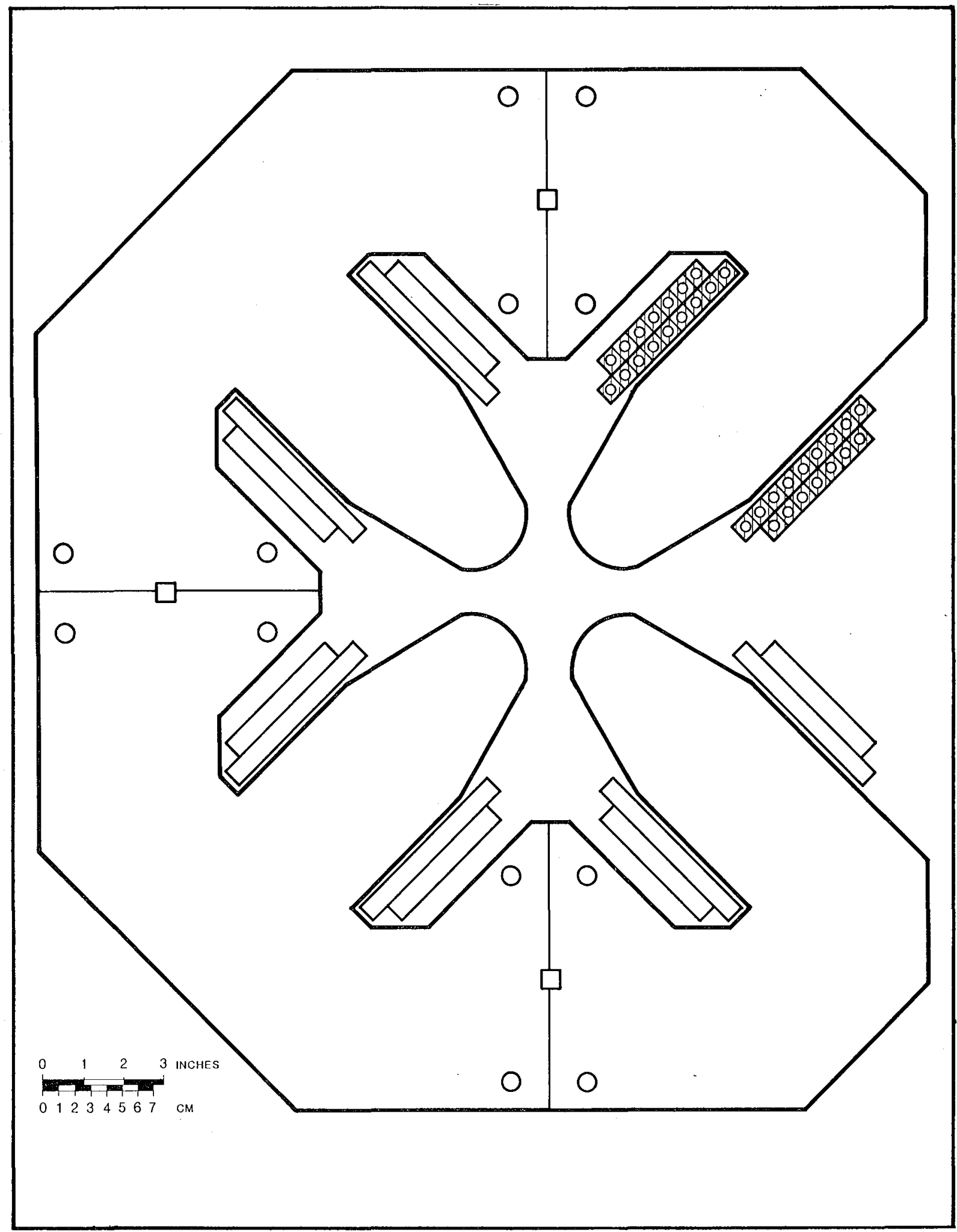

Figure 10. Storage ring quadrupole magnet cross section.

XBL 833-8933A 


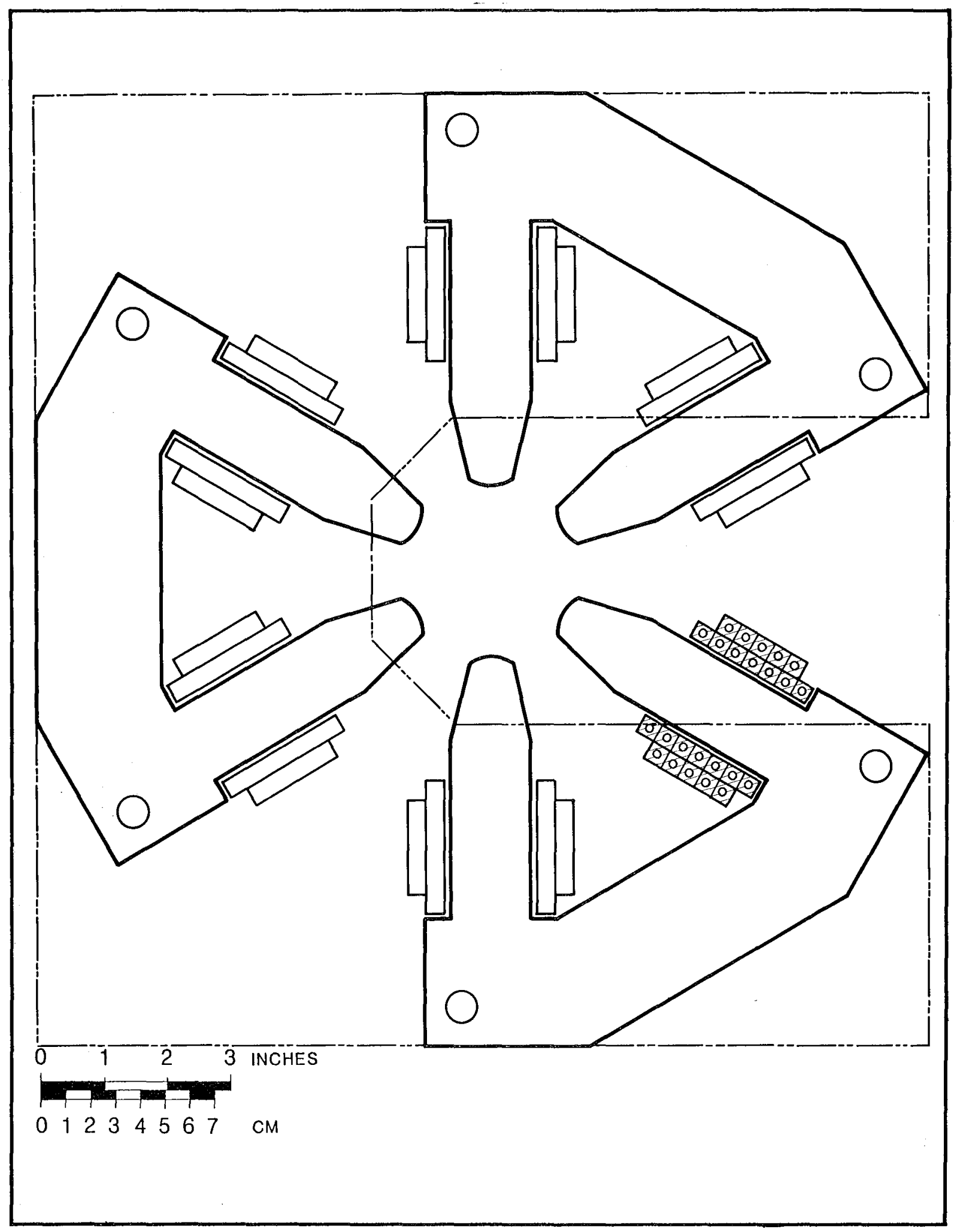

Figure 11. Storage ring sextupole magnet cross section.

XBL 833-8934A 
approximately $28 \mathrm{~cm}$ long. They are designed to accept a horizontally enlarged vacuum chamber, therefore they are constructed in three sectors and are supported in such a manner as to provide complete open access along the outer radius. The poletip field is $0.44 \mathrm{~T}$, and the power dissipation is less than $3.0 \mathrm{~kW}$ per magnet.

Steering magnets are used to correct the closed-orbit errors in the ALS electron storage ring and to adjust the electron-beam position and angle at the insertion devices. Each of the 24 storage-ring dipole magnets is fitted with a separate trim winding for horizontal steering. An additional 24 steering-magnet modules (each with one horizontal steering magnet and one vertical steering magnet) are installed around the storage ring to provide a complete steering capability. Each steering magnet is about $15 \mathrm{~cm}$ long, has a maximum effective field strength of $0.01 \mathrm{~T}-\mathrm{m}$, and has a power dissipation of less than $0.15 \mathrm{~kW}$.

The support and alignment of the magnets are crucial to proper ALS operation. The magnet support system must provide adjustments for three translations and three rotations. These six adjustments are made through three vertical and three horizontal struts. Where practical, several magnets share a common base frame. A multi-magnet package can then be aligned in the laboratory and this prealigned package located in the ring with the six struts. Provision for thermal expansion is provided in all supported elements.

All beam elements, including the linac, the booster ring, the storage ring, and the photon beam lines, must be aligned accurately. To do this, a network of accurately surveyed monuments is strategically placed throughout the ALS area. The horizontal alignment of beam elements is then based upon the monument locations. The vertical alignment of beam elements depends on reference elevations provided by a permanently installed liquid level. This system was developed for the PEP project and is both precise $( \pm 0.10 \mathrm{~mm})$ and quick to use.

Each beam element is located relative to the two nearest survey monuments. Offsets are measured from the line of sight between adjacent survey monuments to each beam element. Elevations are measured relative to the liquid level. A combination of conventional optical tooling instruments and proven distance-measuring devices is used in the gathering of survey data.

Distances between adjacent beam elements and distances to the survey monuments are entered into a small on-line computer for analysis. An important benefit of an on-line computer is the efficient use of survey data. After a group of components has been completely surveyed, the computer is used to calculate the support-stand adjustments which are required. The use of stacks of calibrated shims in the support struts permits these adjustments to be made accurately and with very few iterations. This adjustment scheme was very successfully used at PEP.

\section{Vacuum System}

A vacuum of $10^{-9}$ torr or less is required in the storage ring to obtain adequate electron-beam storage times. Achieving this pressure is relatively straightforward in the absence of stored electrons. However, with a stored electron beam, the dominant gas load is caused by the following two-step process. The synchrotron-radiation photons produce photoelectrons within the surface of the vacuum-chamber wall, and these electrons in turn cause desorption of gas molecules, the most significant of which are $\mathrm{CO}_{2}, \mathrm{CO}, \mathrm{CH}_{4}$, and $\mathrm{H}_{2}$. 


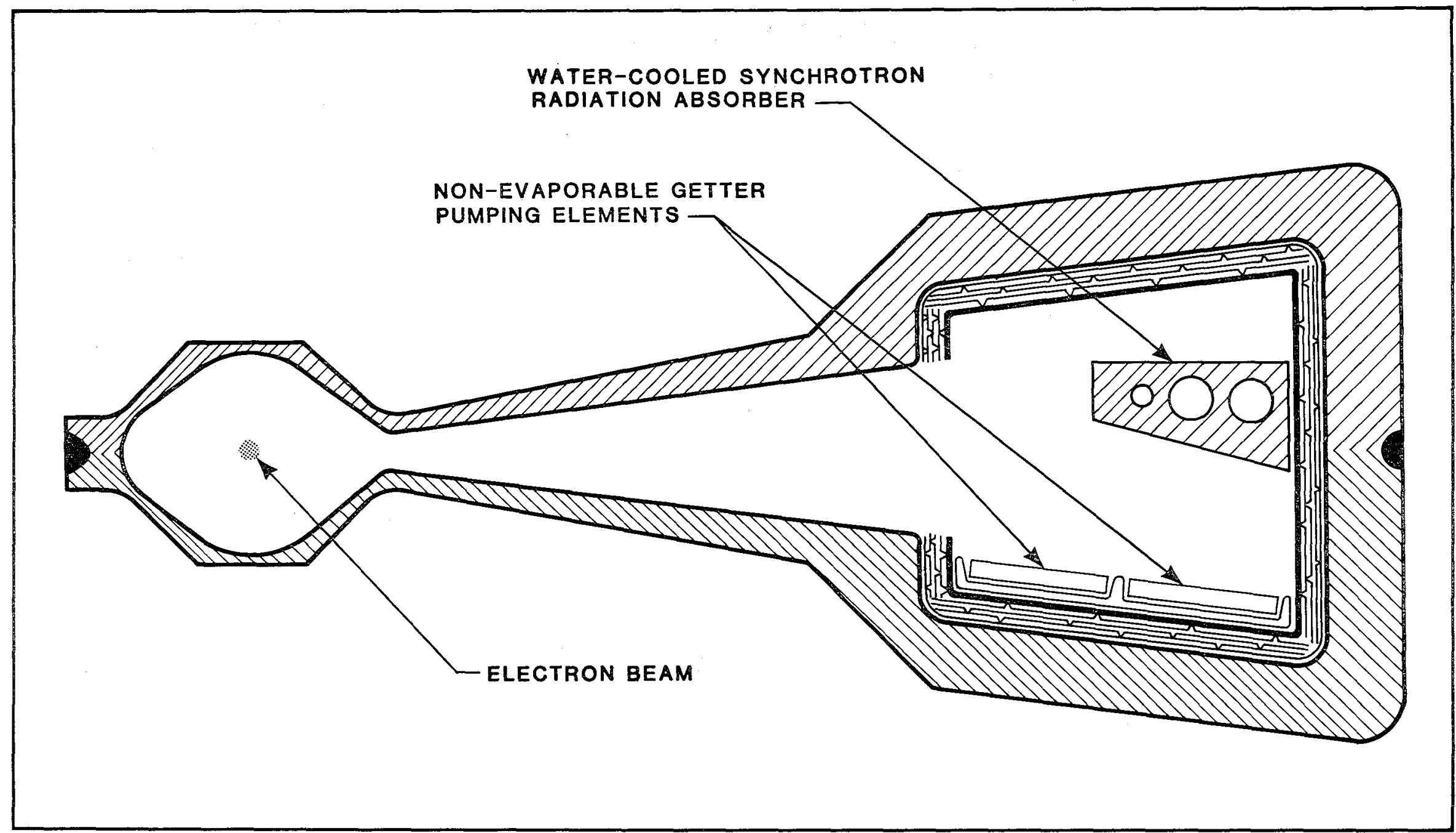

Figure 12. Storage ring vacuum chamber. 
This photon bombardment process has produced copious amounts of gas during initial operation of existing electron storage rings, raising pressures up to $10^{-6}$ torr at full electron beam current. As time passes, the photon bombardment gradually "scrubs" the impinged surfaces, causing the desorption rate to decrease slowly. Typically, it has taken many months to a year of operation for existing storage rings to achieve the desired pressures $\left(<10^{-8}\right.$ torr) and beam lifetime ( $\sim 8$ hours) at full beam current. If similar techniques were used for the ALS, a pressure of $\sim 10^{-8}$ torr could be expected in a few months, but it would take well over a year to reach the vacuum goal of $10^{-9}$ torr. Better performance is clearly needed.

The vacuum-chamber configuration under consideration for the ALS storage ring is shown in Figure 12. The electron-beam circulation region is connected by a long continuous slit to the adjacent pumping chamber which also contains a water-cooled synchrotronradiation absorber.

This design concept provides significantly improved performance, as compared with conventional designs. The residual gas pressure within the vacuum chamber of an operating electron storage ring represents an equilibrium reached between the two competing processes of gas desorption (mainly initiated by the synchrotron radiation) and vacuum pumping. In a conventional design, the gas desorption takes place close to the circulating electron beam, but the distribution pumps are placed in an adjacent chamber. Although the two chambers are connected by small openings which pierce the intervening wall, the restriction of gas flow reduces the effectiveness of the vacuum pump. In the ALS design, in contrast, most synchrotron-radiation photons escape the beam-circulation chamber to strike an absorber in the pumping chamber. Therefore, the gas desorption occurs immediately adjacent to the "non-evaporable getter" (NEG) pumps, and there is no restriction of gas flow. Furthermore, the roominess of the pumping chamber permits the use of large, highperformance pumps. In conclusion, Monte Carlo simulations of molecular flow indicate that the ALS vacuum chamber design should provide at least 100 times improvement in residual gas pressure, when compared with conventional designs. This improvement can be thought of as arising from improved effectiveness of vacuum pumping. It should be noted that this design does not rely upon differential pumping, since there are no separate pumps in the beam-circulation region.

The height of the slit should be large enough to transmit almost all of the synchrotron radiation with photon energies above a few electron volts, so that the gas desorption near the electron beam is minimized. However, the slit should be no larger than necessary, in order to minimize the rf coupling between the two chambers. The useable photons from. the insertion devices and the bending magnets emerge from the storage ring through slits in the outer wall of the pumping chamber. Gas contamination from the photon beam lines would have minimal effect on the circulating beam because of the presence of highperformance pumps in the pumping chamber.

In addition to the NEG distributed pumps described above, appendage pumps with a combined pumping speed of 14,000 liters per second are also included. Oil-free turbo molecular pumps backed by cryogenic absorption pumps are used to "rough" pump the storage ring and to pump it during cleaning by ion glow discharge. 


\section{Magnet Power-Supply System}

The storage-ring and booster power-supply system is based upon the one used at the PEP storage ring. Major magnetic elements (dipoles and quadrupoles) are current regulated by choppers fed from large dc power supplies. The numerous trim and steering requirements are provided by current-regulated bipolar transistor actuators. The reference and monitoring system from the central computer is locally represented by microprocessors interfaced to the current regulators through appropriate $\mathrm{DA}$ and $\mathrm{AD}$ converters. The major magnet currents are controlled and monitored by 16-bit devices and are regulated to an accuracy of $\pm 1 \times 10^{-4}$. The trim currents and steering-magnet currents are regulated to $\pm 1 \times 10^{-3}$ and are controlled and monitored by 12-bit devices.

The storage ring has four families of quadrupole magnets and one dipole-magnet family. Choppers feeding four of these families are all at the same location, each mounted in a single cabinet adjacent to the dc feeder supplies. The two feeder supplies are rated at $200 \mathrm{~kW}$ and $400 \mathrm{~kW}$, with a maximum terminal voltage of $260 \mathrm{~V}$ (see Figure 13).

The ALS choppers are similar to those which have been used successfully at the PEP storage ring. Using a common dc supply minimizes the cost of dc power and allows the use of choppers running at $2.16 \mathrm{kHz}$ and a current-loop unity-gain bandwidth of over a kilohertz. The corresponding high performance of the chopper system has been well documented at PEP. The remaining quadrupole family, Q1, is driven by a $280-\mathrm{A}$ siliconcontrolled-rectifier (SCR) power supply, except for the two quadrupoles, Q1' (in the superconducting wiggler straight section), which will be separately powered. The storage ring also has two families of sextupole magnets, designed for a maximum current of $320 \mathrm{~A}$. These are powered from SCR power supplies. Other SCR supplies are used to power the bending, septum, and quadrupole magnets in the transport line between the booster and storage rings.

The booster ring has three families of quadrupole magnets, two families of sextupole magnets, and one dipole-magnet family. To meet the pulsing rate of $1 \mathrm{~Hz}$ required of the booster, the net voltage needed in the dipole circuit is $2100 \mathrm{~V}$ at a current of $820 \mathrm{~A}$. The $2100-\mathrm{V}$ requirement is best met by four $600-\mathrm{V}$ supplies distributed around the ring, because the voltage above ground can then be limited to $600 \mathrm{~V}$. Only one of the four $600-\mathrm{V}$ supplies needs to be current regulated; the other three will be used as rectification or inversion supplies as required during the rectification, flat-top, and the inversion portions of the pulsing cycle. The current-regulating unit is a chopper, powered from a $630-\mathrm{V}$ dc supply. The three families of quadrupole magnets are each powered with two power supplies in one circuit, in order to provide the desired waveform during the booster cycle. A chopper rated at $260 \mathrm{~V}$ and $1000 \mathrm{~A}$ provides current regulation, and in addition there is a $600-\mathrm{V}$ voltagecontrolled SCR power supply. The dc feeder supplies are rated at $630 \mathrm{~kW}$ and $780 \mathrm{~kW}$, respectively (see Figure 14).

Booster-ring sextupole circuits are rated at $200 \mathrm{~A}$ and $310 \mathrm{~A}$ and are fed from SCR power supplies. The septum magnet, the magnets in the transport section between the linear accelerator and the booster, and the solenoids between the electron source and the linac will all be powered by commercial supplies.

There are numerous steering-magnet supplies used at the ALS. Table 7 lists the supplies needed. 

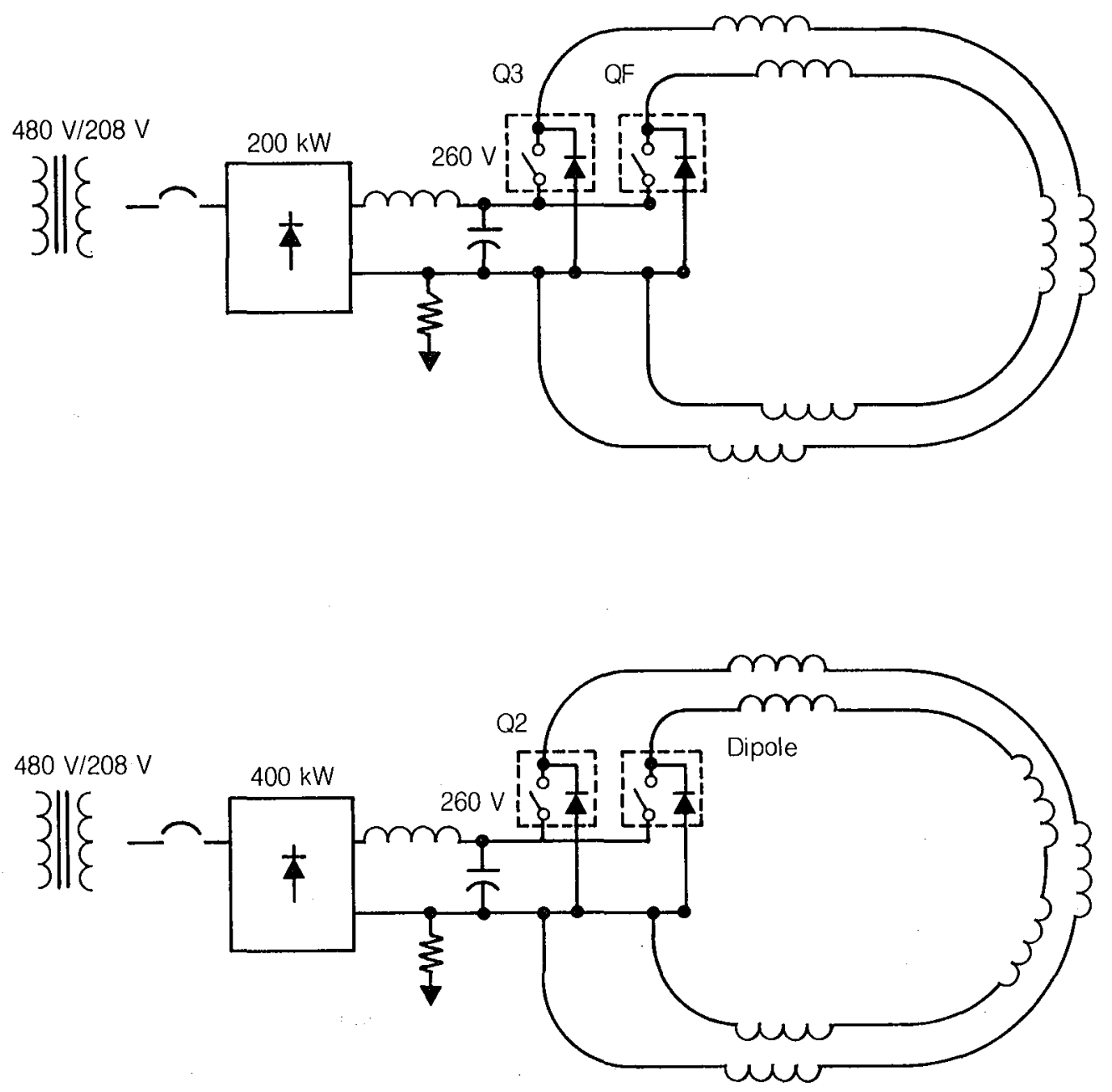

Figure 13. Storage ring magnet power supply.

XBL $833-8936$ 


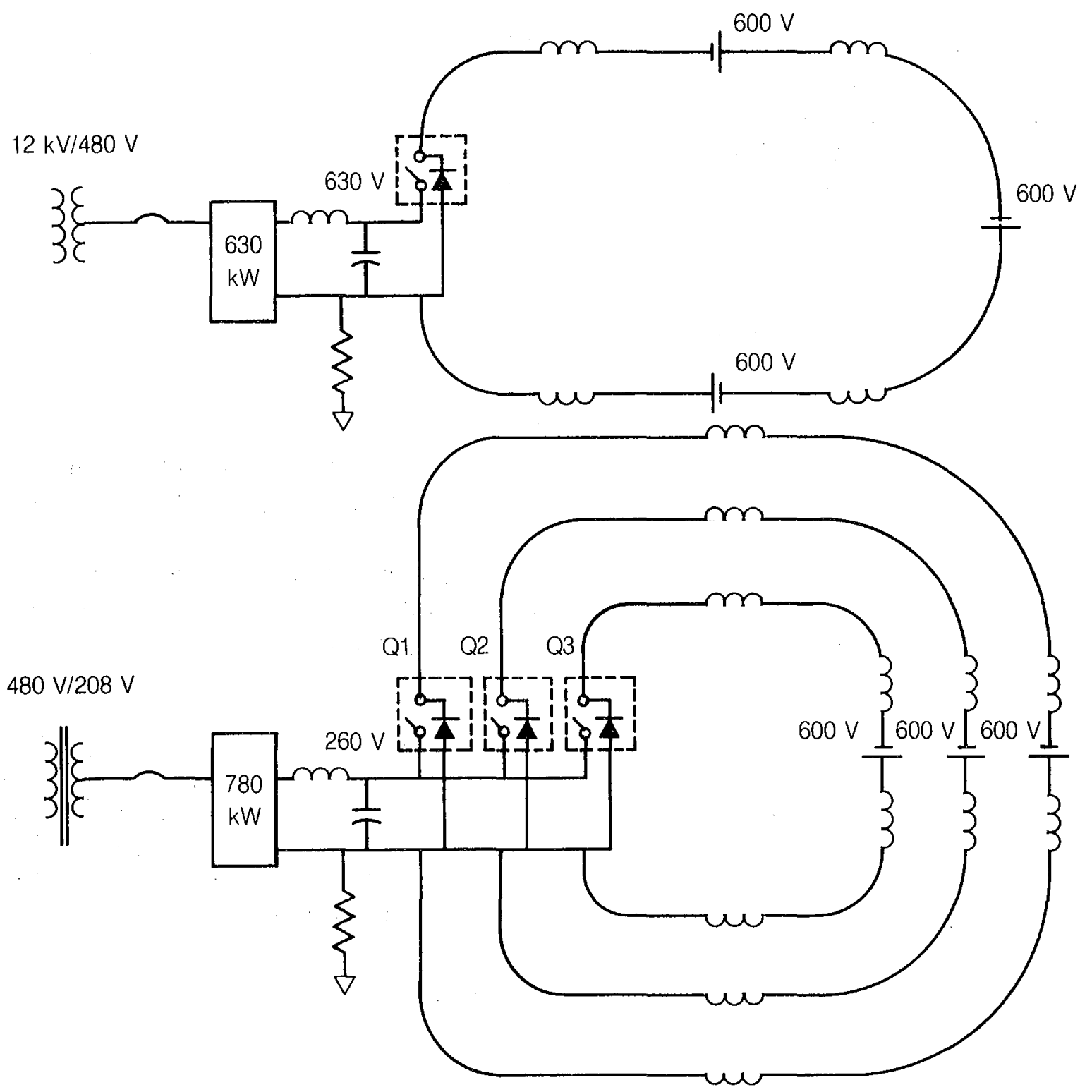

Figure 14. Booster magnet power supply.

XBL 833-8937 
Table 7 .

Required Steering Magnet Power Supplies

\begin{tabular}{llc}
\hline \multicolumn{1}{c}{ Location } & \multicolumn{1}{c}{ Type } & Number of Supplies \\
\hline Storage Ring & vertical & 24 \\
Storage Ring & horizontal & 48 \\
Booster to Storage Ring & vertical & 4 \\
Booster to Storage Ring & horizontal & 1 \\
Booster & vertical & 8 \\
Booster & horizontal & 14 \\
Linac to Booster & vertical & 3 \\
Linac to Booster & horizontal & 1 \\
& & 103 \\
\hline
\end{tabular}

The requirements of all the steering systems can be met by standard SuperHILAC steering-magnet power supplies. These are rated at $100 \mathrm{~W}$ and are packaged with four units in one chassis.

All of the above systems will be provided with appropriate safety interlocks and monitoring devices to protect both personnel and equipment. All of the magnet systems will be grounded through low resistance circuits that can detect ground currents.

\section{RF System}

The functions of the radiofrequency accelerating system in the storage ring are to bunch the beam and to restore the beam energy lost through synchrotron radiation in the wigglers, undulators, and bending magnets. The rf system consists of four cavities driven by klystrons. The cavity gap voltage must be high enough to offset the beam energy loss and to produce very short electron bunches. Table 8 gives some parameters of the rf system for the storage ring. In this table, the total effective shunt impedance is given by

$$
\mathrm{Z}_{\mathrm{eff}}=\mathrm{Z} \mathrm{T}^{2} \mathrm{~L}
$$

where $\quad Z=$ cavity impedance per meter $(\Omega / \mathrm{m})$

$\mathrm{T}=$ transit time factor

$\mathrm{L}=$ cavity length $(\mathrm{m})$.

The fundamental mode cavity dissipation is given by

$$
P=\frac{(V T)^{2}}{Z_{\text {eff }}}
$$

where $\mathrm{V}=$ peak cavity gap voltage

$\mathrm{T}=$ transit time factor. 
Table 8

RF-System Parameters

\begin{tabular}{ll}
\hline Frequency & $499.65 \mathrm{MHz}$ \\
Harmonic Number & 304 \\
Nominal Bunch Length $(2 \sigma)$ & $35 \mathrm{ps}$ \\
Peak Cavity Gap Voltage & $4.4 \mathrm{MV}$ \\
Transit Time Factor & .683 \\
Peak Electron Energy Gain per Turn & $3 \mathrm{MeV}$ \\
Beam Current & $400 \mathrm{~mA}$ \\
Synchrotron Radiation Power & $200 \mathrm{~kW}$ \\
Power Loss to Parasitic Modes & $25 \mathrm{~kW}$ \\
Total Effective Shunt Impedance & $32 \mathrm{M} \Omega$ \\
Fundamental-Mode Cavity Dissipation & $280 \mathrm{~kW}$ \\
Total rf Power Installed & $600 \mathrm{~kW}$ \\
\hline
\end{tabular}

The rf system is divided into two identical rf subsystems. The major components of an rf subsystem (see Figure 15) include two single-cell accelerating cavities, a 300-kW klystron amplifier, a high-voltage power supply and crowbar unit, regulation and control circuitry, and a phase-stable reference line. These will be described below.

The single-cell accelerating cavities have low-loss, semi-spherical designs, and they are very similar to those used at the KEK Photon Factory. The single-cell cavity design was selected because it has fewer potentially troublesome higher order modes, as compared with higher-impedance, multi-cell designs.

The cavities are mounted on the common support girder with the other beamline components. Alignment of a cavity to the beam center line is not critical, since it has only a second-order steering effect on the beam.

Vacuum pumping is provided by a single $100-1 / \mathrm{s}$ ion pump on each cavity. Vacuum monitoring is handled by an ion gauge mounted in the rf feed section.

A constant-temperature cooling-water system minimizes the radiofrequency drift during running periods. The water temperature is controlled by a local-station computer.

The cavity tuners are similar to those developed for the LBL Bevatron RFQ project, except for the addition of a bellows-type seal. During operation, these tuners are automatically adjusted to compensate for reactive beam loading, and they provide stability against phase oscillations.

The cavities are coupled to a WR-1800 aluminum waveguide through a cavity transition piece joined to the wide face of the guide. The vacuum window is in the waveguide section. The gas pressure in the waveguide is maintained at slightly more than atmospheric pressure, and it is carefully monitored. In the event of a loss in pressure (indicative of a waveguide leak), the system is automatically shut down. A WR-1800 Magic Tee is used as a power divider between the two cavities. A waveguide penetration in the concrete shielding completes the connection to the power amplifier.

An rf isolator or automatic load matching tuner may be used in the waveguide between the klystron and the accelerating cavities. The function of this isolator is to maintain a 


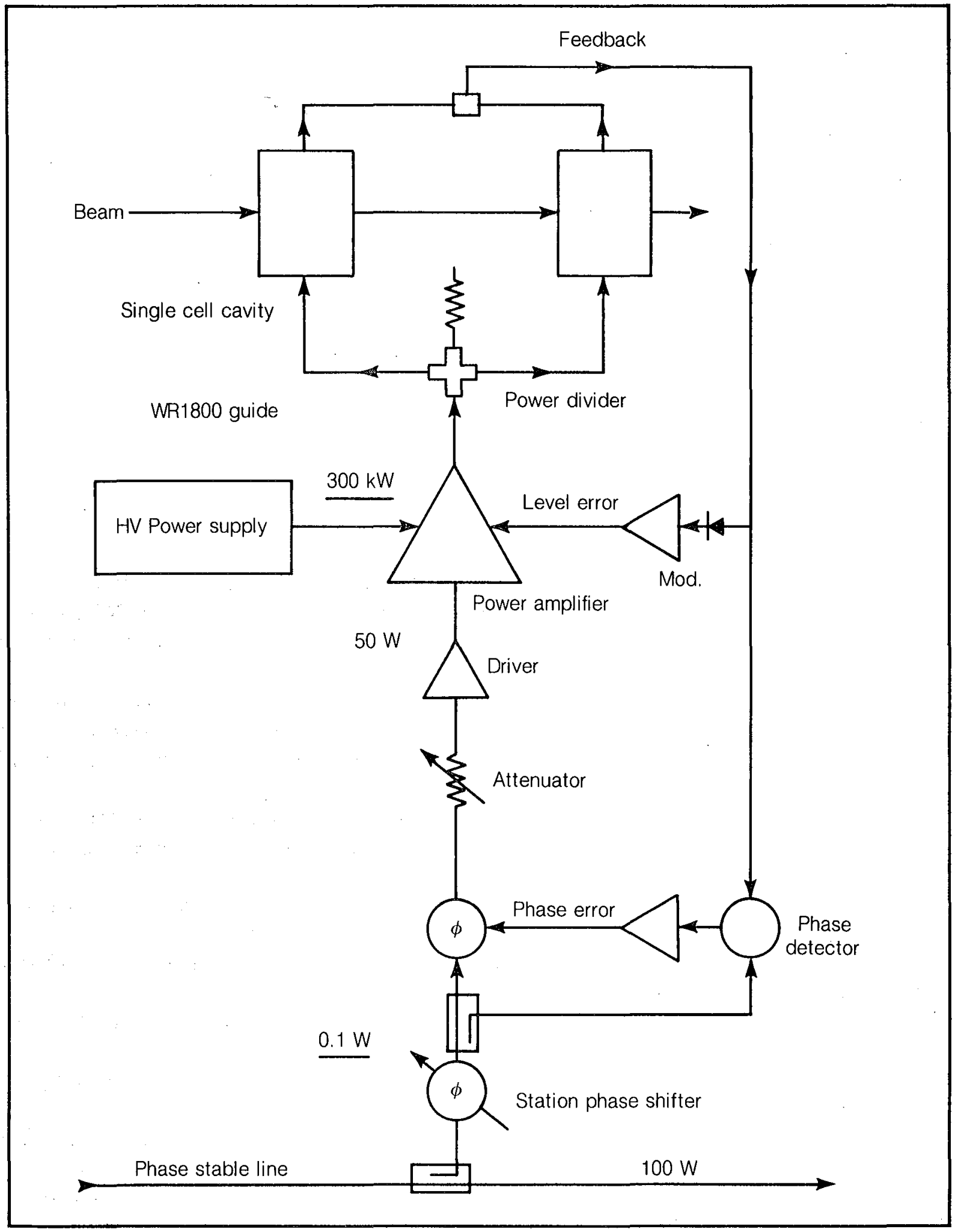

Figure 15. Storage ring RF station.

XBL 833-8938 


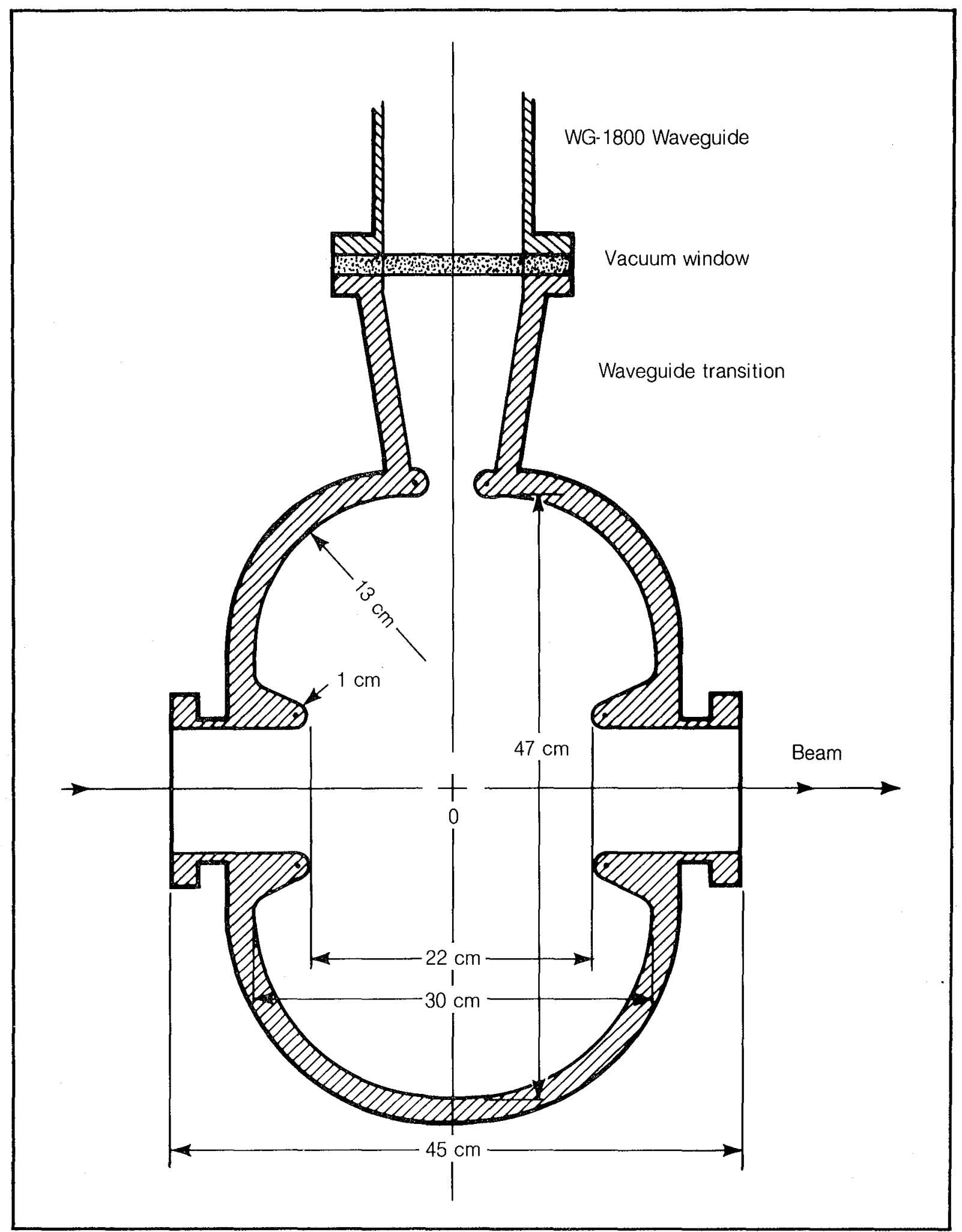

Figure 16. Single cell RF cavity. 
constant load on the klystron for all beam loading conditions at the cavity. The purpose is to prevent klystron damage and to enhance system stability.

The power for acceleration is provided by a Varian VKP-8259 klystron amplifier (located outside the shielded area for ease of tuning and maintenance). The VKP-8259 delivers up to $300 \mathrm{~kW}$ of $\mathrm{CW}$ output power at $500 \mathrm{MHz}$, and the tube has a modulating anode for ease of control. Klystron beam power required is $45 \mathrm{kV}$ at $15 \mathrm{~A}$, which is supplied by a standard pad-mounted power supply and crowbar unit. The beam voltage is controlled by a variable voltage transformer (VVT) and vacuum contactor located adjacent to the power supply. The VKP-8259 requires only about $50 \mathrm{~W}$ of drive for fully saturated output, and this can easily be obtained from a standard communications-type solid-state amplifier.

Proper operation of the storage ring requires accurate control of the relative phases and the amplitudes of the output of the two radiofrequency subsystems. A controller chassis very similar to the one developed for the LBL Uranium Beams Project controls the relative phases to \pm 1 degree and the amplitude to \pm 1 percent.

All accelerator and experimental areas are served by a phase-stable reference system, composed of a stable master oscillator operating at $499.65 \mathrm{MHz}$ followed by a $100-\mathrm{W}$ amplifier. Each drop from the stable reference cable is by a $30-\mathrm{dB}$ coupler, which prevents any one user from causing system errors in the event of equipment malfunction. A user is thus provided with a very stable rf signal phase-locked to the circulating beam in the accelerator. Other timing signals (see the following section) are also transmitted to allow the user to identify any individual radiofrequency bucket.

All parameters involved with personnel safety (e.g., high-voltage barriers) are controlled with a hardware interlock chain. All other system-performance information is collected and processed by a local controller, the Input Output Micro Module (IOMM) described in the section on the control system. This IOMM performs such important functions as adjusting the VVT to minimize energy consumption, monitoring the klystron temperature profiles, and ensuring proper system performance. Additionally, there is a fast crowbar controller to detect klystron-window and gun arcs and remove all power within microseconds to prevent equipment damage. The local IOMM also monitors the performance of the crowbar controller and can turn off the high voltage in the event that its operation is unsatisfactory.

\section{Ring Low-Level Electronics}

In designing the ALS facility, great care has been taken to provide the capability to monitor properly and to control the beam and the various components of the accelerator itself. For the first task, instrumentation such as Faraday cups, current transformers, beam position-monitoring electrodes and synchrotron-light monitors are required, whereas the second task requires the use of vacuum monitoring equipment and various computercontrolled synchronization and monitoring devices. In addition to those systems directly concerned with accelerator operation, there are other categories, such as communications and safety-related systems, which also play an important role in the running of a large accelerator complex. Each of these systems is described briefly below.

Wall-current monitors composed of resistors shunting a gap in the beam tube are used to measure beam intensity and position in the injector linac and transfer lines. Signals from these monitors are available in the control room for observation on fast oscilloscopes. 
The position signals are processed to generate $\mathrm{x}$ and $\mathrm{y}$ positions normalized to beam intensity. A computer reads these data and generates a display of beam position for the operator.

A high-frequency Faraday cup in the beam line between the linac and booster is used to determine beam intensity and time structure. A similar cup is used in the transfer line between the booster and storage ring. Suitable electronics for using the cup signals as input to a computer are included.

Beam position and beam size at several locations in the linac and transport lines are monitored with scintillators and TV cameras. Television monitors in the control room are used to display the scintillator images.

Average beam intensity in the booster and storage ring is measured with current transformers surrounding a metallized ceramic portion of beam pipe. The metallic layer is thin enough to permit the lower-frequency components of the circulating beam to couple to the transformers.

At 16 locations in the booster and 84 locations in the storage ring, beam-position monitoring electrodes are installed. Each monitor consists of four button-electrodes, electrostatically coupled to the beam. Signals from the electrodes are processed to obtain the beam center-of-charge. The resulting $x-y$ position signals are sent to a small computer where the beam positions in the booster and the storage ring may be scanned quickly to determine the closed-orbit distortions of the electron beam. Fast signals from selected buttonelectrodes are also available in the control room for display on a high-frequency oscilloscope.

Synchrotron-light monitors are installed at the booster and storage rings. These consist of photomultiplier tubes, fast photodiodes, and TV cameras. A suitable mirror directs the visible portion of the synchrotron light to the monitors through quartz viewing ports, while the bulk of the synchrotron light passes through the mirror to a photon beam dump. The synchrotron-light monitor is especially useful for detecting low levels of beam, calibrating beam transformers, and detecting beam structure.

Electrodes and driver amplifiers are installed in the booster and storage rings to permit excitation of beam resonances. Signals from position electrodes in the rings are sent to a spectrum analyzer in the control room so that beam instabilities may be studied.

Ion pumps on the storage ring are energized by multichannel power supplies in equipment racks distributed around the ring. Ion gauges at 36 locations around the storage ring are used to measure ring pressure. The ion-gauge power supplies are scanned by a computer so that a profile of ring pressure can be generated. To protect the storage ring vacuum, a system of fast pressure sensors and fast valves is installed at the photon ports. Ion gauges are also used to monitor the pressure in the injector system.

The booster magnetic field, electron source, linac rf, bunchers, and kickers are synchronized such that any number of bunches from 1 to 304 may be accumulated in the storage ring. To accomplish this, a special timing system is provided with inputs from the $500-\mathrm{MHz}$ reference system, the booster magnetic field, and the beam-position monitors in the booster and the storage ring. The stored bunches are "tagged" to allow the injection system to stack a specified number of them. A particular injection scenario is loaded into the control computer via a touch panel, and the computer then sets up the timing system for the appropriate synchronization. Experimenters are also provided with synchronizing pulses so that they can anticipate the arrival of a photon burst. Main-control-room 
oscilloscopes are provided with synchronizing pulses to enable observation of beam- and machine-related signals.

Real-time observation of beam-related signals is essential for accelerator operation. Very-fast oscilloscopes are installed in the control room for such work. Spectrum analyzers are used to monitor rf parameters, whereas relatively low-frequency beam instabilities are monitored with wave-form analyzers or low-frequency spectrum analyzers. Lowrepetition-rate signals, such as the booster guide field, are displayed on a digitizing oscilloscope. TV monitors and video switches are also provided for observation of synchrotronlight and scintillator images.

As mentioned above, the communications system plays an important role in the smooth operation of the ALS facility. The overall system consists of a local ALS public address system and a Laboratory-wide public address system, which allow voice communications to any point in the facility, and a headset intercom system. All three systems are powered from an emergency generator to prevent loss of communications during a power outage.

The ALS headset intercom system will be especially useful during the installation of the magnets, vacuum systems, and other accelerator-system components. This system includes 24 stations with 12 channels available at all stations. Locations are distributed throughout the ALS facility including the storage-ring, injector, insertion-device, and photon-beam-line areas and the control room. Conversations are duplex, with provisions for any number of stations to be on a given channel while other conversations are being carried on simultaneously.

The ALS safety system is a multi-purpose system designed to ensure that personnel are not exposed to ionizing radiation, non-ionizing radiation, electrical shock, or other hazards. In addition, it provides protection against damage to equipment. The system consists of a pair of redundant, hardwired, fail-safe electro-mechanical circuits connected to controlled devices via an enclosed (and locked) cross-connect system. All semiconductors used are redundant and self-checking. The system is on emergency power to ensure uninterrupted interlock-status information. System status is available to the computer control system, but the computer will not be able to override any fault condition. In the event of an unexpected entry into a protected area, the following equipment will be turned off:

all magnet power supplies

all rf power supplies

all inflection power supplies

all extraction power supplies

all electron-source power supplies

Normal entry, except for well-controlled variances, requires that all of the above be in the safe condition.

Other personnel-safety features include emergency "crash-off" boxes located not more than 100 feet apart. These "crash-off" boxes will cut all ALS power, except for emergency power. 


\section{Injection System}

The injection system for the ALS consists of a 50-MeV electron linear accelerator and a $1.3-\mathrm{GeV}$ booster synchrotron. High-energy injection into the electron storage ring was chosen to minimize for experimenters any possible interruptions caused by the filling process. Injection at the normal operating energy will help to avoid small, but disruptive, changes in the beam-spot positions that can be produced by energy ramping.

\section{Booster}

The booster synchrotron accepts the $50-\mathrm{MeV}$ beam from the linac, accelerates it to 1.3 $\mathrm{GeV}$, and transfers it to the storage ring. The booster cycles at $1 \mathrm{~Hz}$, each cycle consisting of a 0.35 -second accelerating ramp, a 0.2 -second flat top for allowing the beam to damp completely, a 0.35 -second descending ramp, and a 0.1 -second quiescent period.

The booster can be operated in several modes. For the many-bunch mode of operation in the storage ring, 92 of the $112 \mathrm{rf}$ bucket are filled, leaving a 40-ns gap during which the injection kicker magnet turns off and the extraction kicker magnet turns on. In this mode the filling time of the storage ring to $400 \mathrm{~mA}$ is about 2.75 minutes assuming a 20$\mathrm{mA}$ beam from the $50-\mathrm{MeV}$ linac, single-turn injection into the booster, and a $50 \%$ overall transfer efficiency.

For the few-bunch modes of operation in the storage ring, the linac is pulsed to produce a series of 21 short (2-ns) but intense (40-mA) beam pulses spaced at 32-ns intervals. A three-turn mode of injection is used to inject these bunches into 7 of the 112 rf buckets in the booster. During the 0.2 -second flat top of the booster cycle, all 7 of the loaded buckets can be sequentially transferred to just one of the buckets in the storage ring; a waiting period of approximately one damping period $(25 \mathrm{~ms})$ must be allowed between transfers. This transfer scheme is a principal reason for choosing the booster-to-storage-ring circumference ratio of $7 / 19$.

To load the storage ring to $400 \mathrm{~mA}$ in this few-bunch mode takes 289 booster cycles (assuming an overall transfer efficiency of $50 \%$ ), or 4.8 minutes. Since each booster cycle can be individually timed and the seven beam bunches can be distributed in the storage ring in a number of ways, a large number of "few-bunch" modes of operation are possible in the storage ring.

The lattice of the booster synchrotron is shown in Figure 17. It is similar to the lattice of the storage ring in that it is made up of a series of achromatic cells with intervening long straight sections. There are six such achromatic cells, and the six long long straight sections are each 2.4 meters long. The circumference is 67.2 meters $(7 / 19$ of the storage ring circumference). This lattice was chosen over several others that were studied because it has the following favorable properties: convenient injection and extraction; low emittance; low dispersion; high superperiodicity; adequate space for sextupoles, beam monitoring equipment, and correction magnets; and efficient use of space. These properties tend to minimize the injection- and extraction-magnet requirements, the required apertures, and the required rf voltage. They also permit a convenient location of the booster within the existing building.

The major parameters of the booster synchrotron are given in Tables 9 and 10 . 


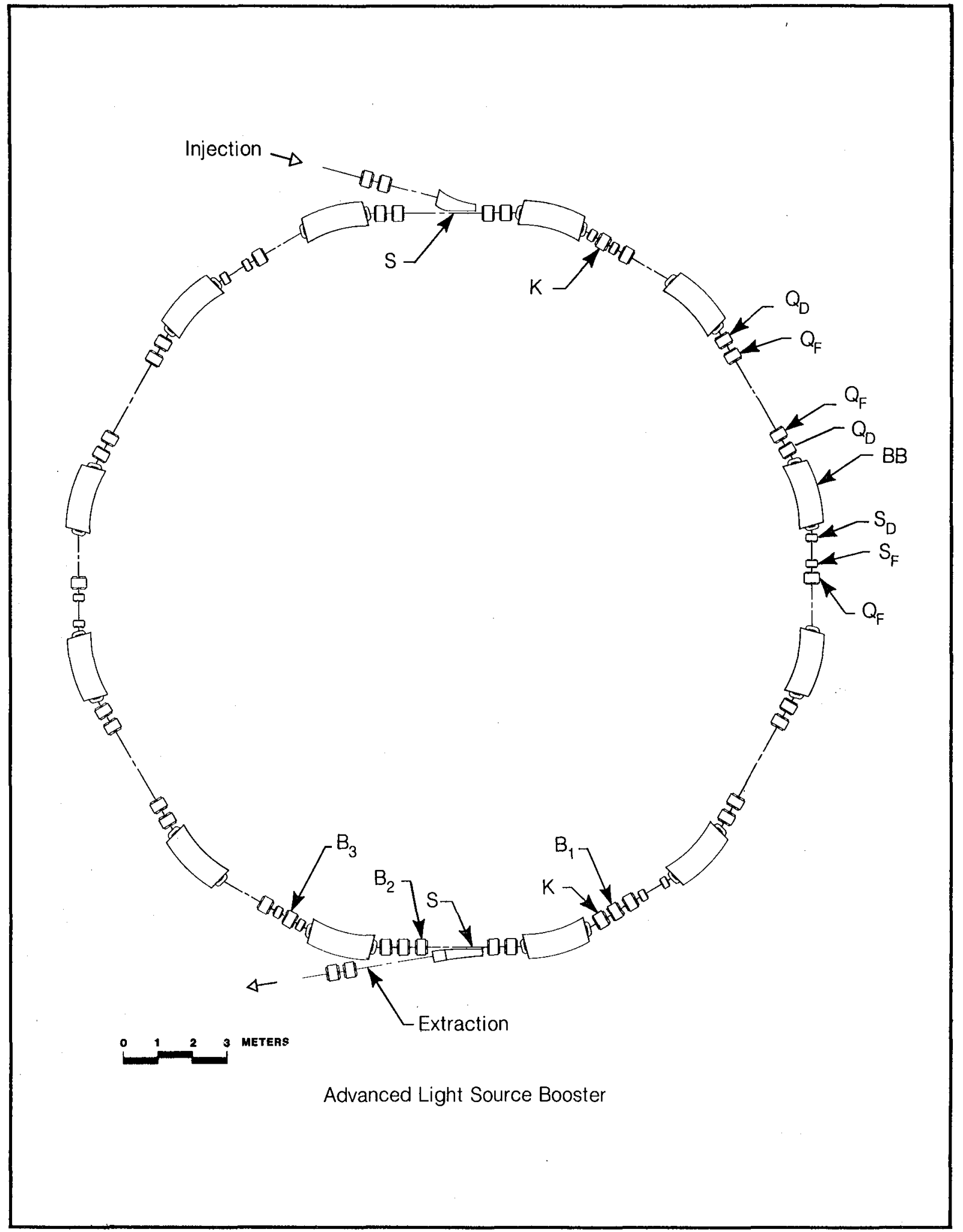

Figure 17. Booster synchrotron. 
Table 9

Booster Synchrotron Design Parameters

\begin{tabular}{lc}
\hline Electron Energy (GeV) & $0.5-1.3$ \\
Electron Current, Many-Bunch Mode (mA) & 13 \\
Electron Current, Few-Bunch Mode (mA) & 7 \\
Cycle Rate (Hz) & 1 \\
Beam Particles & $1.8 \times 10^{10}$ electrons \\
Circumference (m) & 67.2 \\
No. of Superperiods & 6 \\
Horiz. Emittance, 1.3 GeV ( $\pi$ m-rad) & $7.5 \times 10^{-8}$ \\
Energy Spread, 1.3 GeV & $\pm 0.060 \%$ \\
Dipole Bending Radius (m) & 3.53 \\
Dipole Length (m) & 1.85 \\
Max. Dipole Field (T) & 1.227 \\
Quad. Length (m) & 0.28 \\
Max. Quad. Gradient (T/m) & 13.64 \\
Vacuum Aperture, dipoles (cm) & $4 \times 5.5$ \\
Vacuum Aperture, quadrupoles & $10 \mathrm{~cm} \mathrm{diameter}$ \\
Max. Horiz. Beta (m) & 11.22 \\
Max. Vert. Beta (m) & 15.47 \\
Horizontal and Vert. Tune & $4.83,1.88$ \\
Horizontal and Vert. Chromaticity & $-7.35,-5.98$ \\
Momentum Compaction & 0.0149 \\
Revolution Frequency (MHz) & 4.46 \\
Radiofrequency (MHz) & 499.65 \\
Harmonic Number & 112 \\
Max. RF Voltage, peak (kV) & 200 \\
Synchrotron tune, $v$ & 0.0062 \\
Energy Gain per Turn (keV) & 0.81 \\
\hline & \\
\hline
\end{tabular}


Table 10

Booster Parameters at Different Energies

\begin{tabular}{lcc}
\hline & $50 \mathrm{MeV}$ & $1.3 \mathrm{GeV}$ \\
\hline Radiation Loss per Turn & $0.16 \mathrm{eV}$ & $72 \mathrm{keV}$ \\
Energy Spread $(\Delta \mathrm{E} / \mathrm{E})$ & $\pm 7.5 \times 10^{-3}$ & $\pm 6.0 \times 10^{-4}$ \\
RF Bucket Half-Height $(\Delta \mathrm{E} / \mathrm{E})$ & $\pm 3.9 \times 10^{-2}$ & $\pm 6.4 \times 10^{-3}$ \\
Damping Times: & & \\
$\quad$ Horizontal & $141 \mathrm{~s}$ & $8.0 \mathrm{~ms}$ \\
$\quad$ Vertical & $144 \mathrm{~s}$ & $8.2 \mathrm{~ms}$ \\
$\quad$ Energy & $72 \mathrm{~s}$ & $4.1 \mathrm{~ms}$ \\
\hline
\end{tabular}

The booster synchrotron magnet system includes 12 bending magnets, 30 quadrupole magnets, 12 sextupole magnets, and 24 steering magnets (12 horizontal, 12 vertical). The booster bending magnets have a laminated-yoke construction to minimize eddy current problems and to maintain good field penetration and field quantity during the acceleration ramping of the injection cycle. The booster ring generally requires larger magnet apertures than does the storage ring but does not require access through the bending magnets for synchrotron radiation beam ports.

The bending magnets will be of an " $H$ " type construction with flat pancake coils encircling the poles. The " $\mathrm{H}$ " shape will be punched as a single piece from the steel lamination to produce good magnetic field uniformity and good structural rigidity. Coils will be sized to fit through the magnet gap for insertion during magnet assembly. The magnets will be curved to follow the beam trajectory and will have an effective magnetic length of $1.85 \mathrm{~m}$. The magnet cross section is shown in Figure 18. The nominal operating parameters of the dipoles for booster operation at $1.3 \mathrm{GeV}$ are shown in Table 11. 


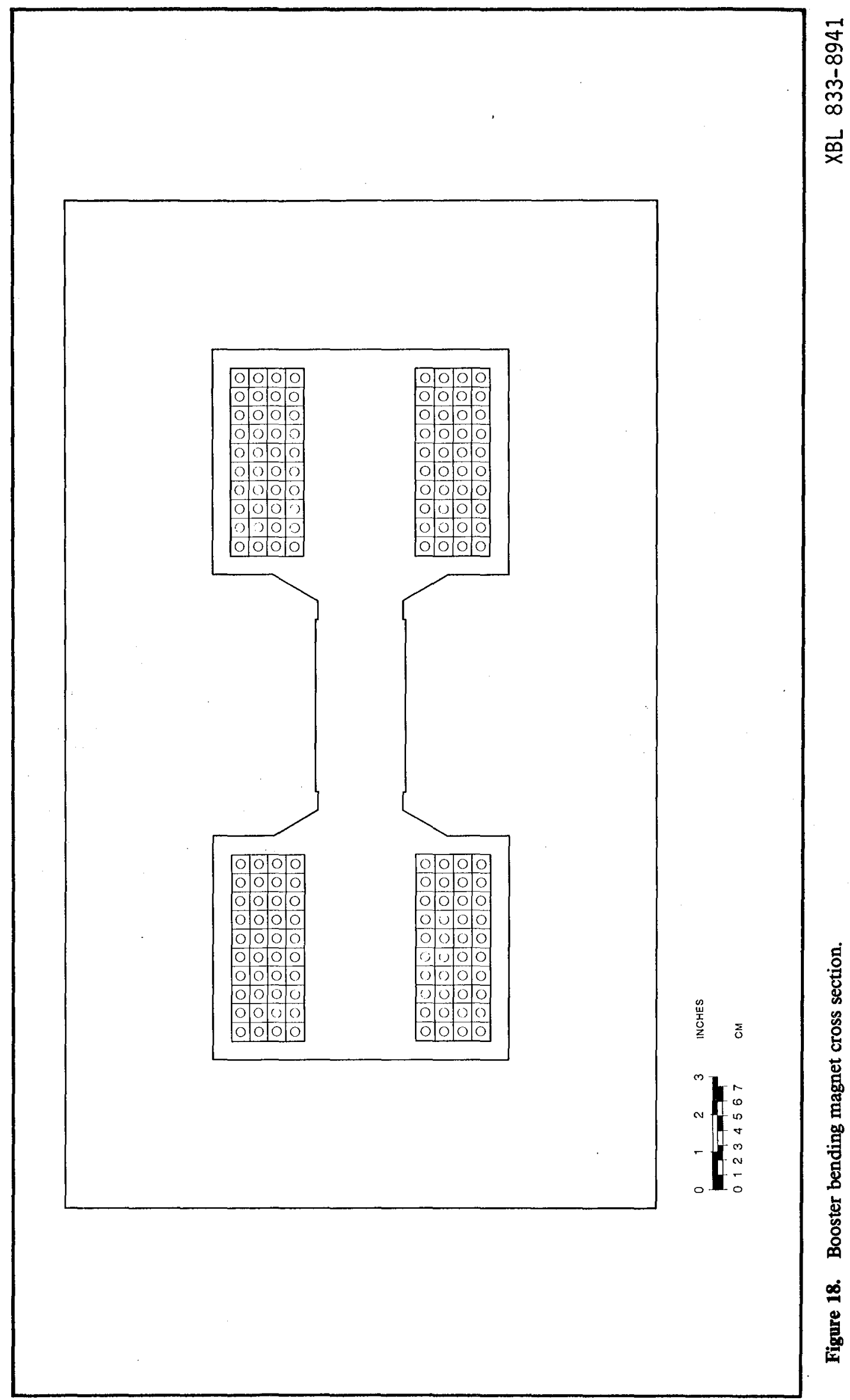


Table 11

Booster Dipole-Magnet Parameters

\begin{tabular}{lc}
\hline Number Required & 12 \\
Bending Angle (degrees) & 30 \\
Field (T) & 1.23 \\
Gap Height (cm) & 6.0 \\
Good-field Width (cm) & 5.0 \\
Length, Magnetic (m) & 1.85 \\
Stored Energy (J) & $1.65 \times 10^{4}$ \\
Inductance (H) & $5 \times 10^{-2}$ \\
Resistance ( $\Omega$ ) & 0.0665 \\
Current, Peak (A) & 818 \\
Voltage, dc (V) & 54 \\
Voltage, ac, Peak (V) & 115 \\
\hline
\end{tabular}

The quadrupole magnets have an effective length of $28 \mathrm{~cm}$ and a maximum design gradient of $17.7 \mathrm{~T} / \mathrm{m}$. The design uses a split-yoke assembly with essentially parallel-sided coil slots, as shown in Figure 19. A single-piece lamination is not attractive here, because it does not allow adequate clearance for coil insertion through the aperture during assembly and requires very difficult coil fabrication procedures. Using a smaller coil is unattractive because of the need for very-high current densities in the conductors. The space between the coils along the horizontal midplane provides convenient clearance for the deflected electron beam in the extraction channel. Furthermore, the quadrupole side yoke can be easily modified to provide even greater horizontal beam space, if required. The operating parameters for each of the three quadrupole families are given in Table 12. 


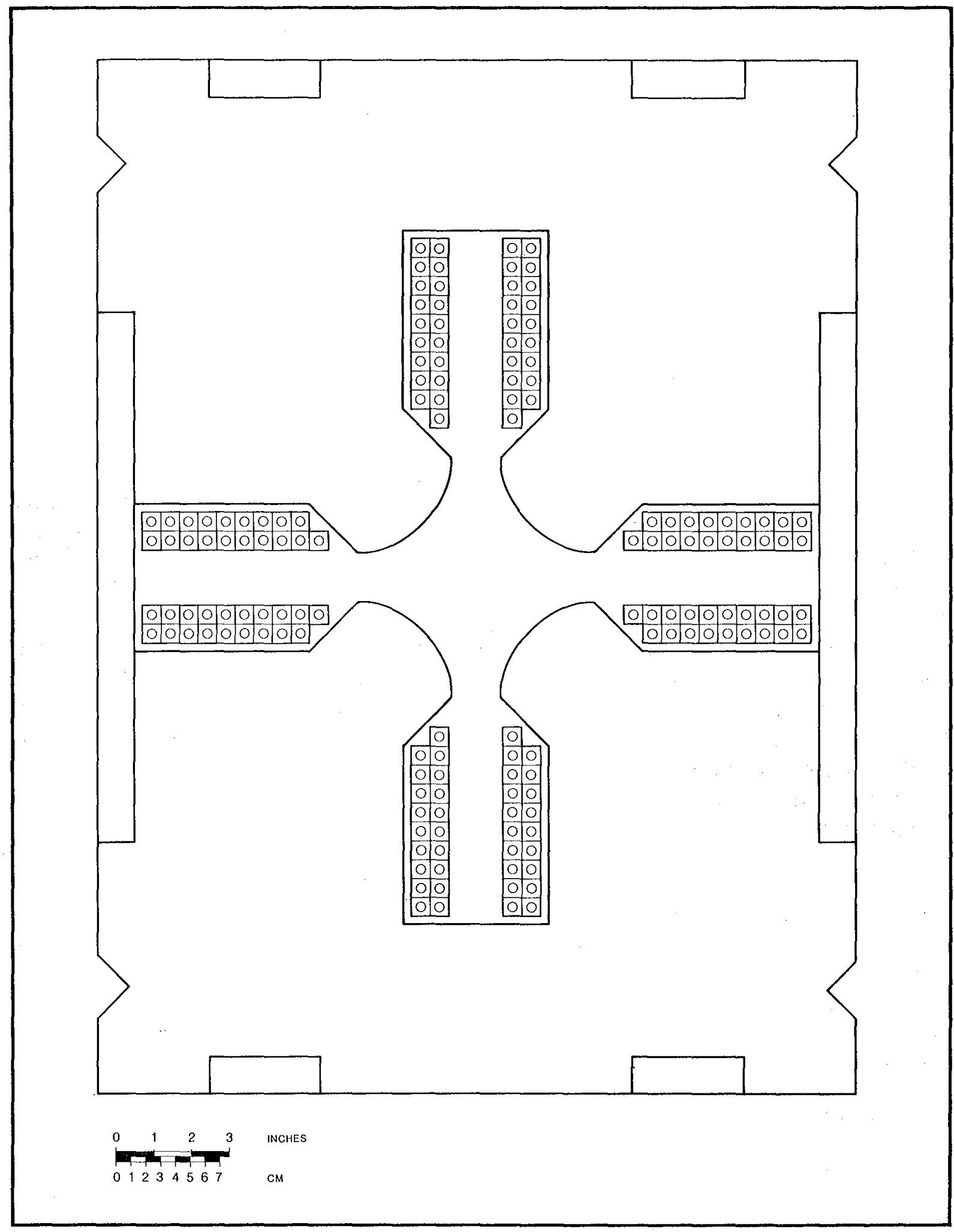

Figure 19. Booster quadrupole magnet cross section.

XBL $833-8942$ 
Table 12

Booster Quadrupole Parameters

\begin{tabular}{lccc}
\hline Quadrupole ID & QF1 & QD2 & QF3 \\
Number Required & 12 & 12 & 6 \\
Aperture Diameter (cm) & 10.0 & 10.0 & 10.0 \\
Effective Length (cm) & 28.0 & 28.0 & 28.0 \\
Maximum Gradient (T/m) & 16.8 & 15.4 & 17.7 \\
Current (A) & 900 & 830 & 960 \\
Voltage, dc (V) & 14 & 13 & 15 \\
Power, dc (kW) & 13 & 11 & 15 \\
\hline
\end{tabular}

The booster sextupole and steering magnets are of similar construction to those of the storage ring (described in the Section on Ring Magnets). There will be a total of 12 sextupoles and 24 steering magnets in the booster lattice. A booster sextupole magnet is shown in Figure 20.

The booster vacuum chamber consists of 1-mm-wall-thickness stainless steel tubing, except in the injection and extraction regions, where ceramic sections are required. The relatively modest vacuum requirement of $1 \times 10^{-7}$ torr in the booster ring can be obtained with four $220-1 / \mathrm{s}$ ion pumps distributed around the booster. The transfer line between the booster and the storage ring has a pressure gradient of $10^{-7}$ to $10^{-9}$ torr, but its relatively small diameter and great length make it possible to maintain this gradient with a 100-1/s ion pump. An isolation valve separating the two rings is needed for booster maintenance and for protection against vacuum accidents.

The pulsed kicker magnets and the narrow septum magnets are described in the following Section on the Beam Transfer System. Similarly, the booster-magnet power-supply system is described in the Section on the Magnet Power-Supply system.

The rf system for the booster synchrotron uses a single if resonator of construction similar to that of the main-ring resonators. The few $\mathrm{kW}$ of rf power is easily supplied by a commercial UHF transmitter, modified to our requirements. The phase- and gradientcontrol system is identical to that used for the storage ring. The rf signal is fed from the phase-stable reference system.

\section{Beam Transfer System}

The beam transfer system for the ALS consists of the following subsystems: $50-\mathrm{MeV}$ beam transport from the linac to the booster; single-turn and multi-turn injection into the booster; beam extraction from the booster; $1.3-\mathrm{GeV}$ beam transport from the booster to the storage ring; and injection into the storage ring.

Beam transport of the $50-\mathrm{MeV}$ electron beam from the linac to the booster requires three bending magnets and four quadrupoles. The beam line that transports the $1.3-\mathrm{GeV}$ beam between the booster and the storage ring requires four bending magnets and seven quadrupoles. The quadrupole design is the same as that for the storage-ring quadrupoles. 


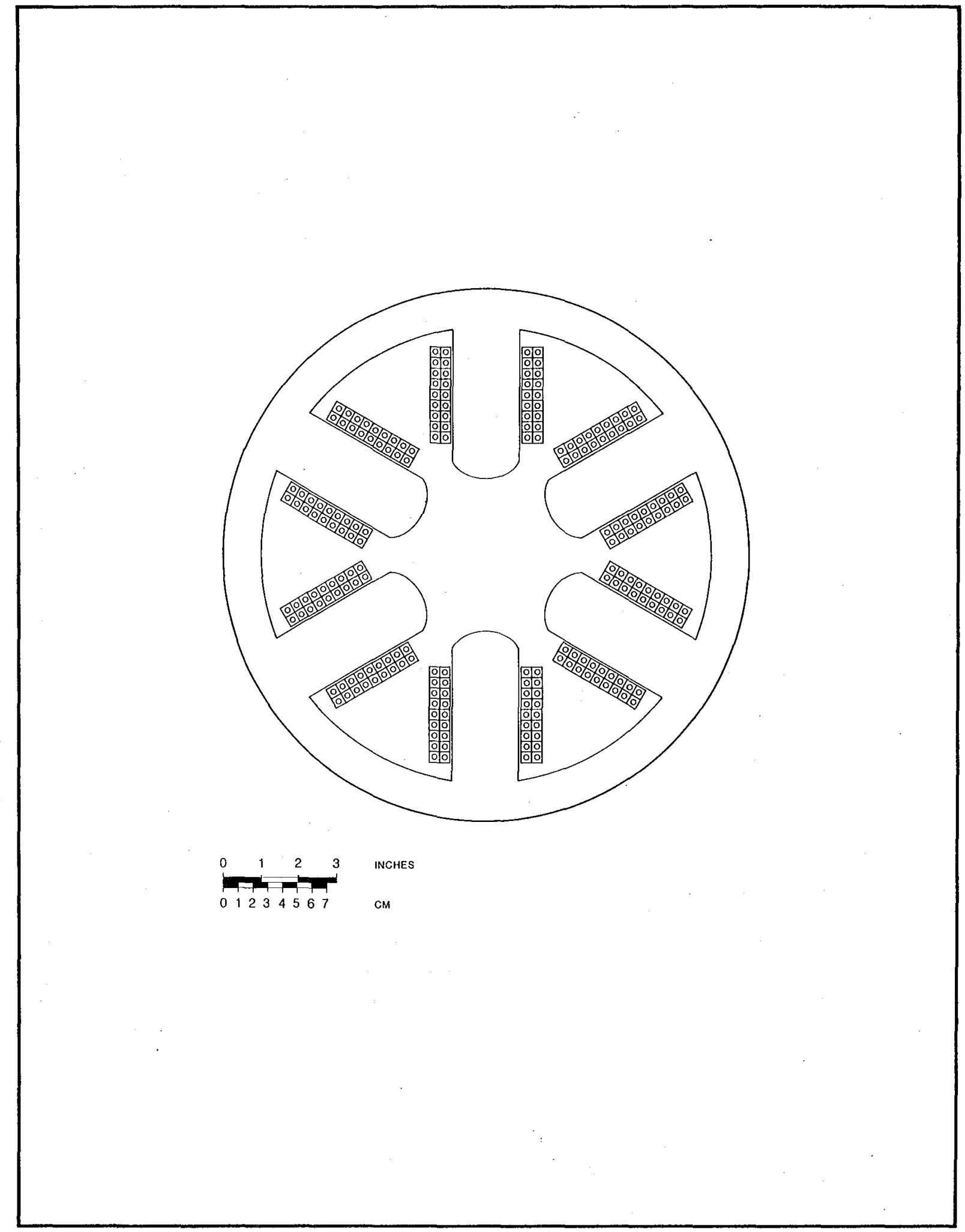

Figure 20. Booster sextupole magnet cross section. 
Beam injection and extraction from the booster synchrotron are accomplished using similar components in opposite straight sections of the booster. The injector uses a septum magnet and a fast kicker for single-turn injection (see Figure 21). The same septum magnet, along with three bump magnets, is required for three- or four-turn phase-space stacking of the injected beam in the booster ring. These bump magnets are excited with a current waveform that rises to full field and decays in roughly stepwise fashion to zero during four revolution periods in the booster. This mode of stacking in betatron phase space works well if the fractional part of the betatron tune is approximately 0.25 . Figures 22 and 23 show details of the kicker magnet; Figure 24 shows a septum magnet; and Figure 25 shows a bump-magnet power supply.

Beam extraction from the booster (see Figure 26) requires a fast kicker, three bump magnets, and two septum magnets. The three bump magnets establish an offset equilibrium orbit for roughly 100 revolution periods. When the bump-magnet current reaches its peak, the fast kicker steps the beam across a narrow copper septum. In the many-bunch mode, the kicker risetime is less than $40 \mathrm{~ns}$ to fit into the 40-ns gap in the booster beam. In the few-bunch mode, the kicker rise and fall times must be less than $32 \mathrm{~ns}$, the spacing between the seven filled buckets. After extraction the beam is deflected beyond the yoke of the last quadrupole in the straight section by a thicker magnetic septum. Two septum magnets are required because of current-density limitations in the thinner septum.

Bucket-to-bucket transfer of the electron beam bunches from the booster to the storage ring is made possible by using the same radiofrequency $(500 \mathrm{MHz})$ in the two rings. In the many-bunch mode, the beam bunches in the 92 filled buckets of the booster are transferred to 92 of the 304 buckets in the storage ring. The consecutive transfer cycles are randomly synchronized, so that eventually the filling of all the rf buckets in the storage ring becomes reasonably uniform.

In a typical transfer cycle, the electron beam being injected into the storage ring is launched into a horizontal coherent oscillation with an amplitude that is much larger than the width of the beam already stored. (Because transverse damping times are about $25 \mathrm{~ms}$ in the storage ring, the stored beam has time between injection cycles to damp to its equilibrium size.) The equilibrium orbit of the stored beam is moved to within $4 \sigma_{\mathrm{x}}$ of the injection septum when the injected beam is launched on its initial trajectory. A half-wave bump is used to make this shift in the equilibrium orbit. A fractional horizontal tune near $1 / 4$ or $3 / 4$ is used. Consequently, to avoid having the injected beam hit the septum during the fourth revolution, the half-wave bump must be turned off in $2.2 \mu \mathrm{s}$. This is a standard method of injection into electron storage rings. Details of the storage ring injection system are shown in Figure 27.

The booster fast kickers for injection and extraction require fall times for injection and rise times for extraction of approximately $40 \mathrm{~ns}$ in the many-bunch mode of operation. The flat tops for both these magnets are approximately $185 \mathrm{~ns}$ long. In the few-bunch mode, the same extraction kicker magnet is used but with a different modulator to provide a faster risetime and a shorter pulse. The three injection bump magnets and three extraction bump magnets for the booster ring and the three injection bump magnets for the storage ring are pulsed with roughly half-sine waveforms a few microseconds long. All the fast kickers and bump magnets have ferrite cores, wound with one-turn current-sheet conductors. In the case of the fast kickers, the modulators are mounted adjacent to the magnet. All the pulsed magnets are powered separately, and the currents of these magnets must 


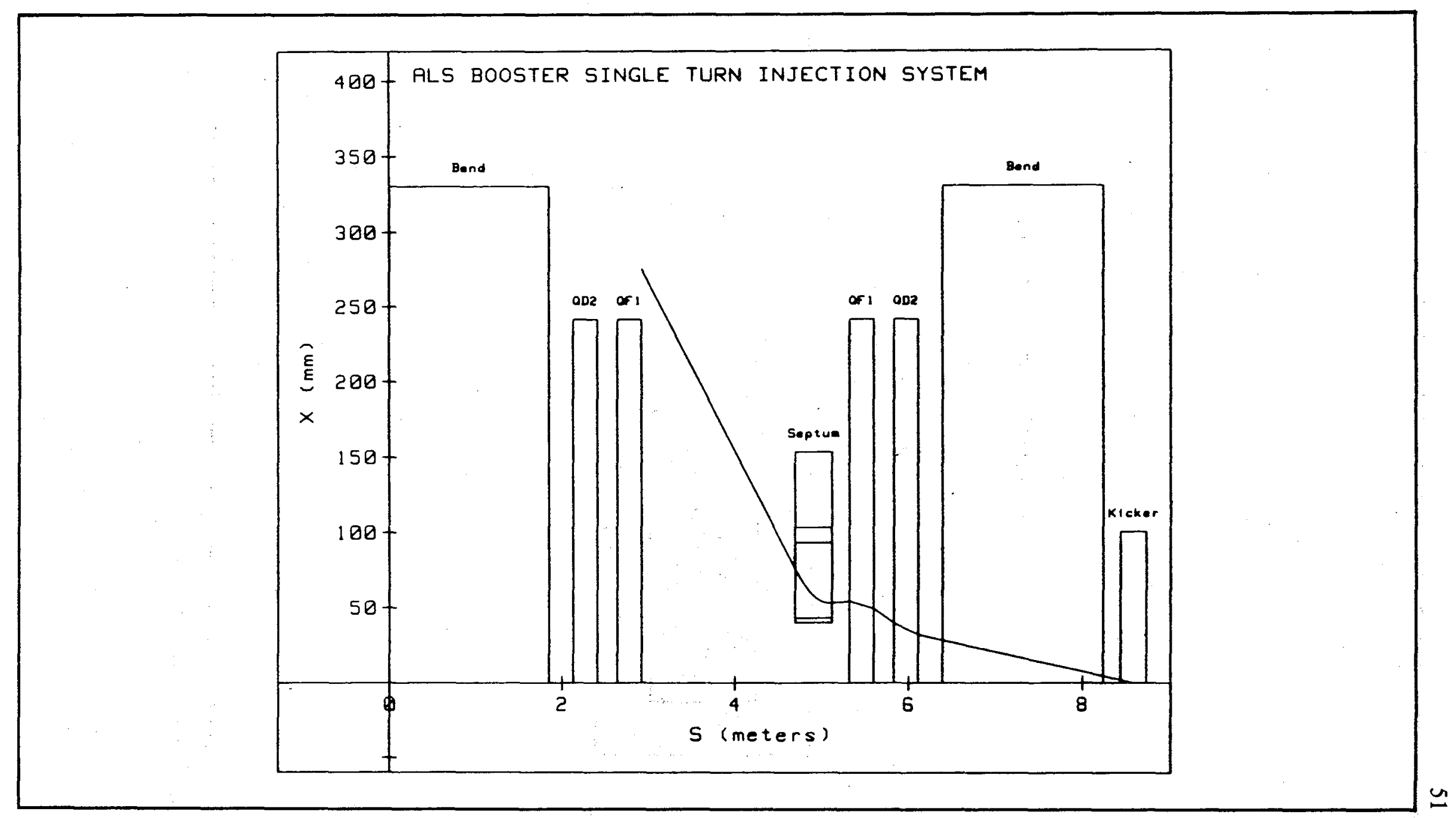

Figure 21. Booster beam injection system. 


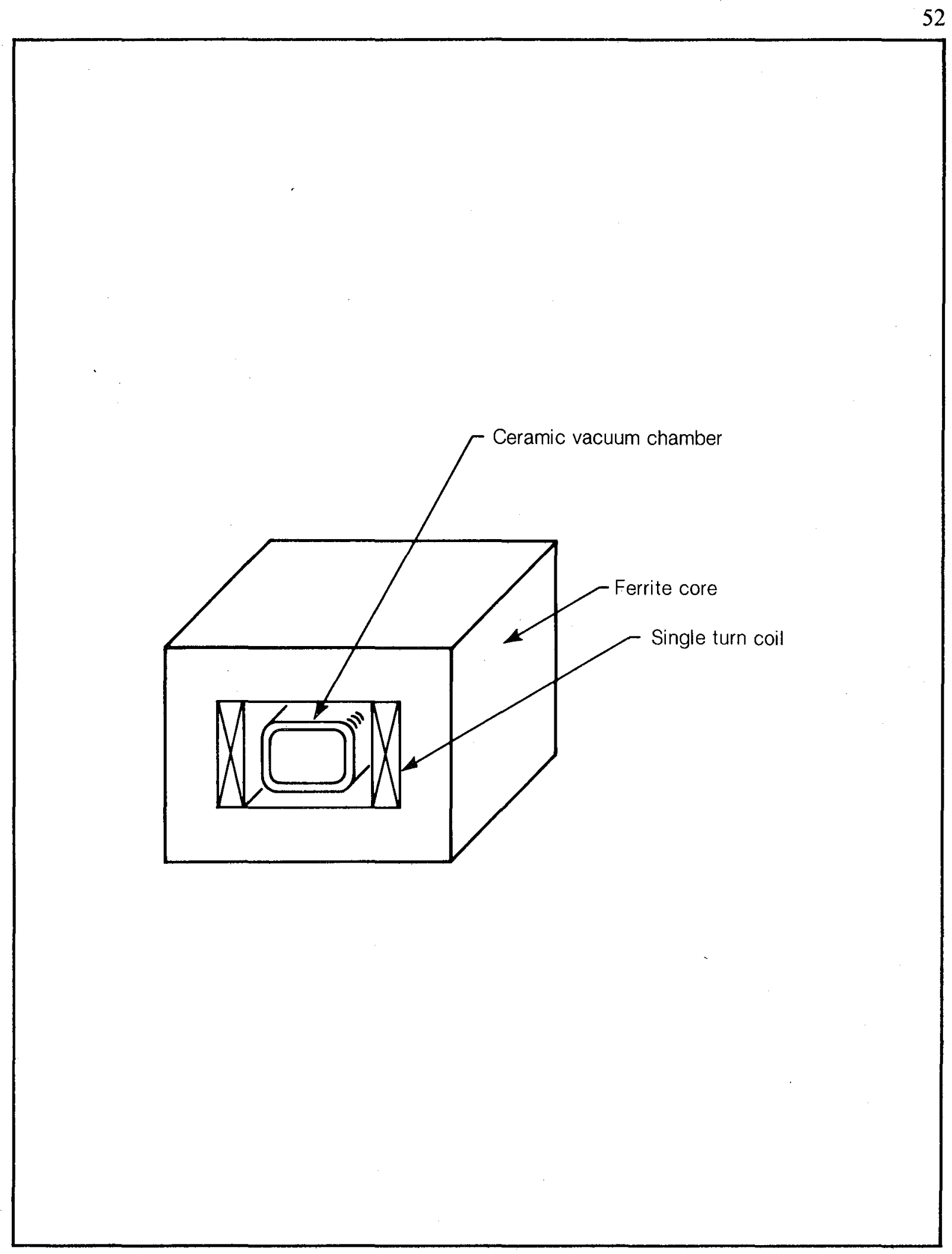

Figure 22. Kicker magnet.

XBL 833-8945 


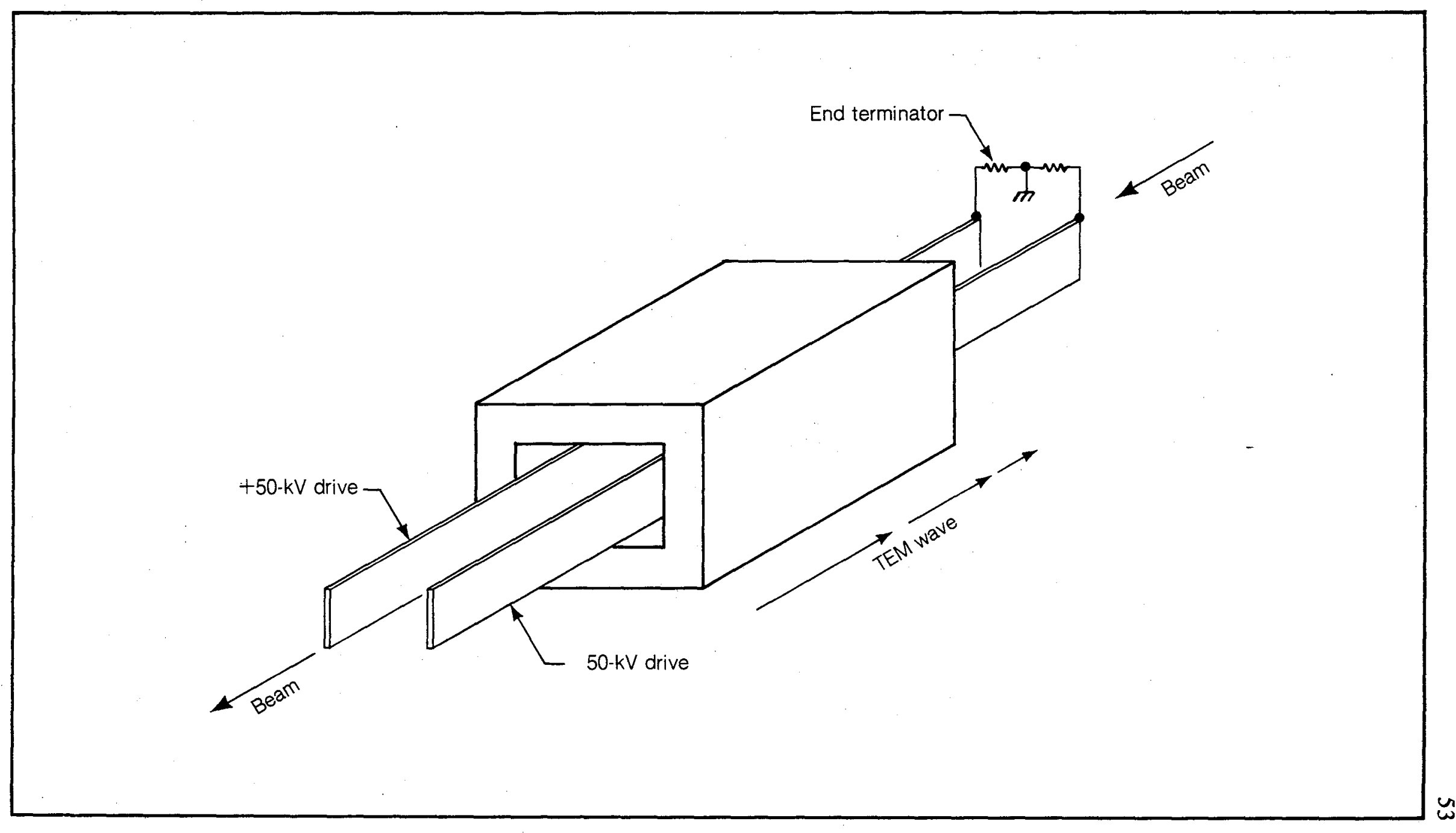

Figure 23. Fast beam kicker ferrite-loaded strip-line magnet. 


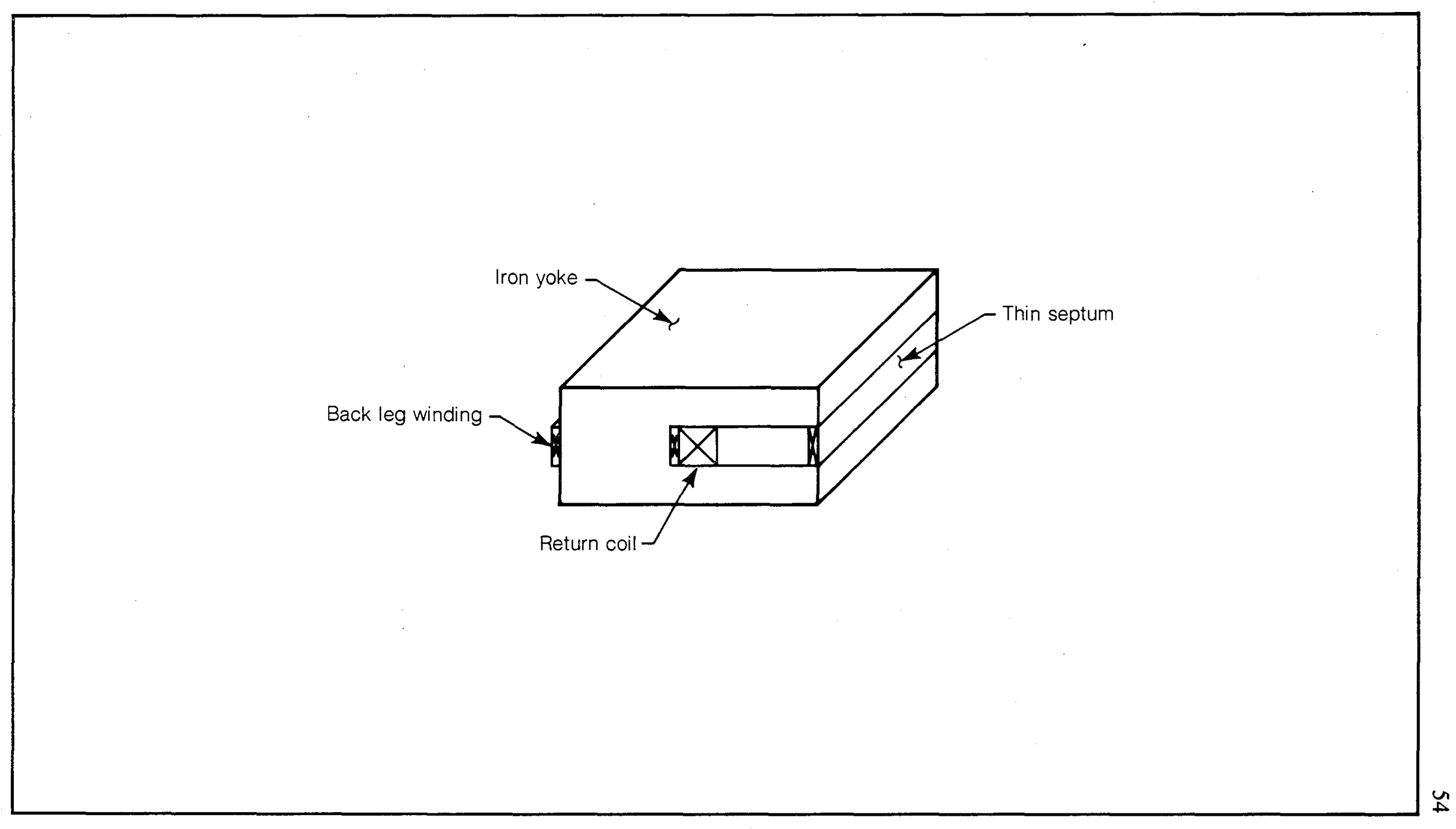

Figure 24. Injection/extraction septum magnets.

XBL 833-8947 


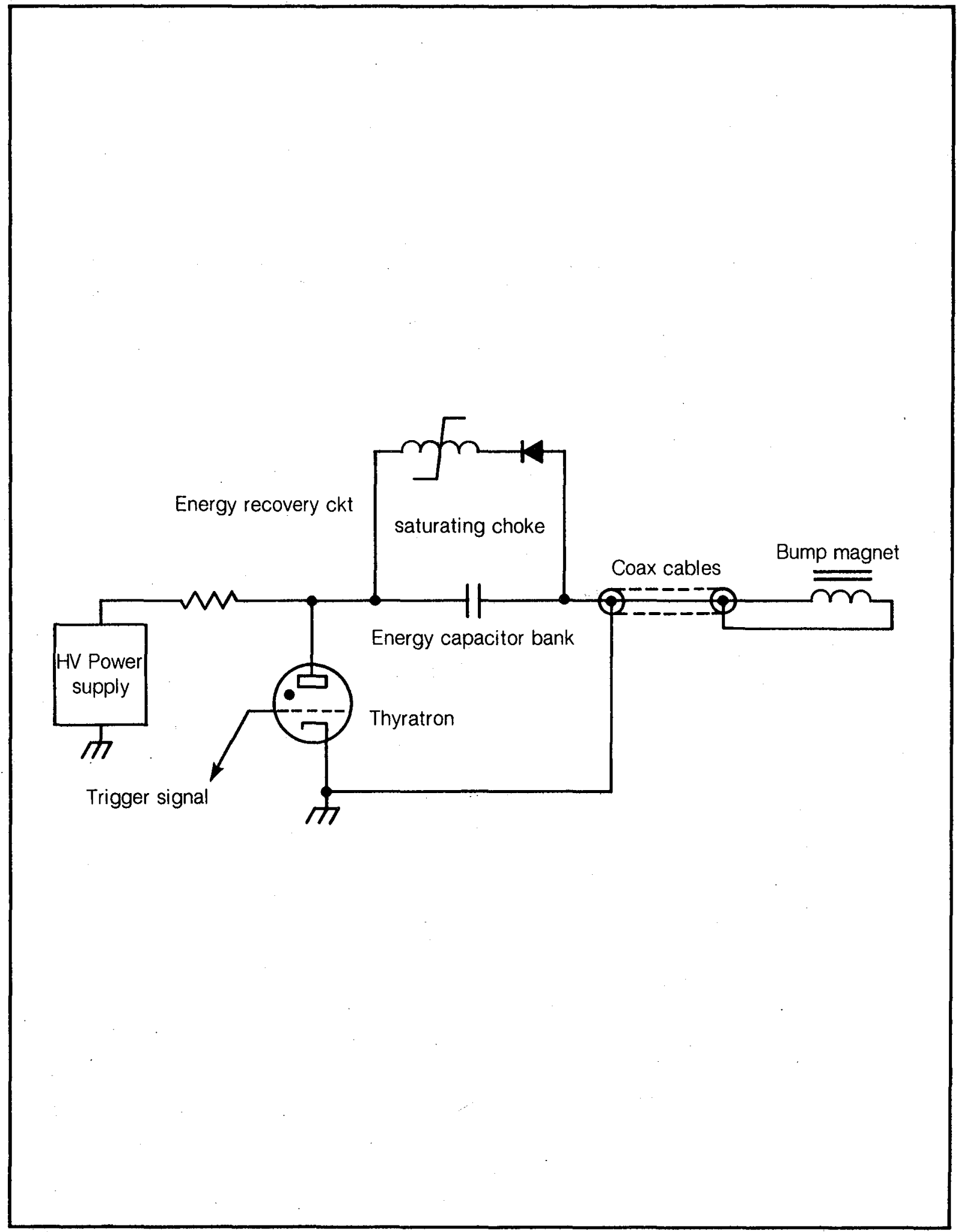

Figure 25. Medium-speed "beam bump" magnet power supply.

XBL $833-8948$ 


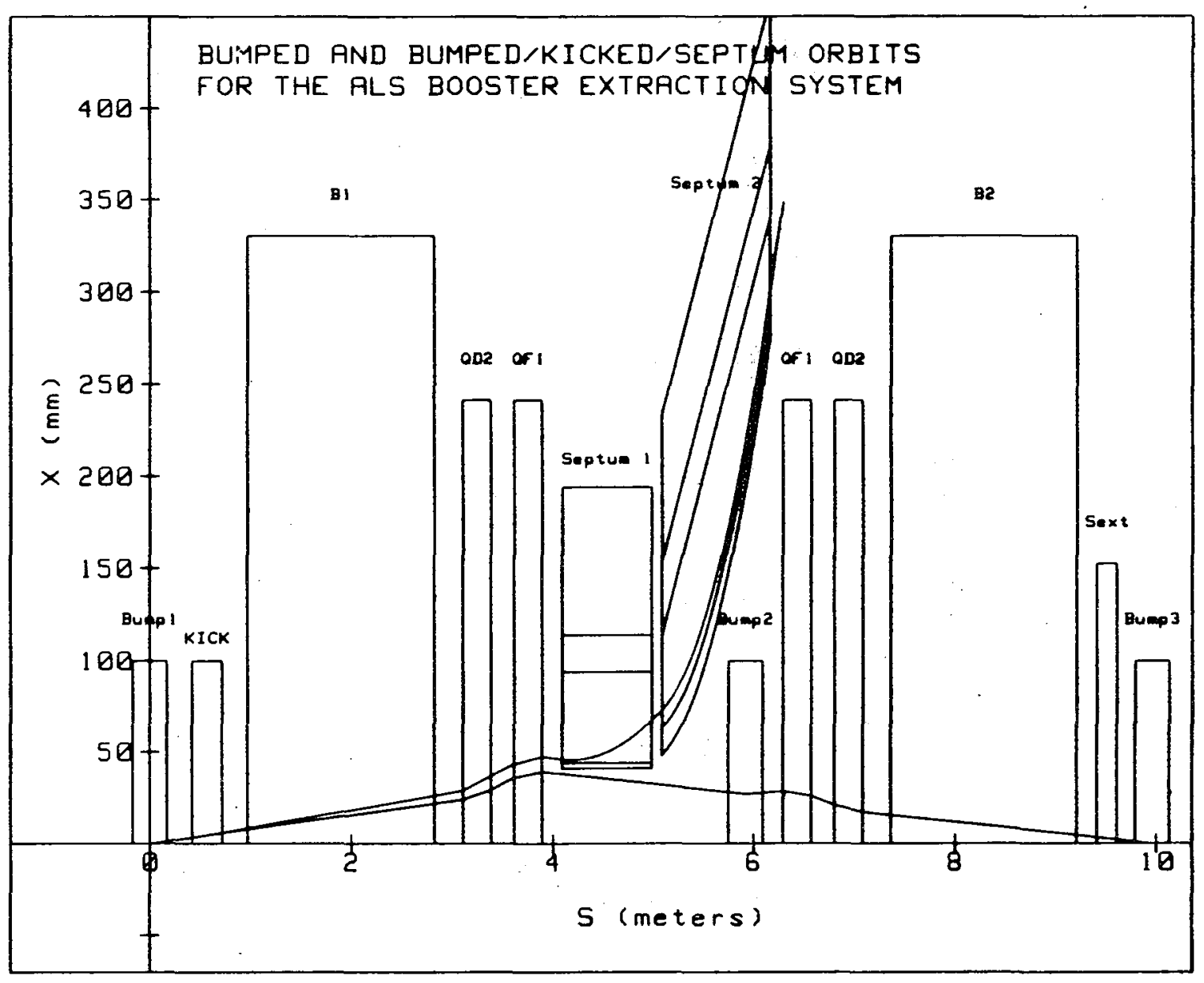




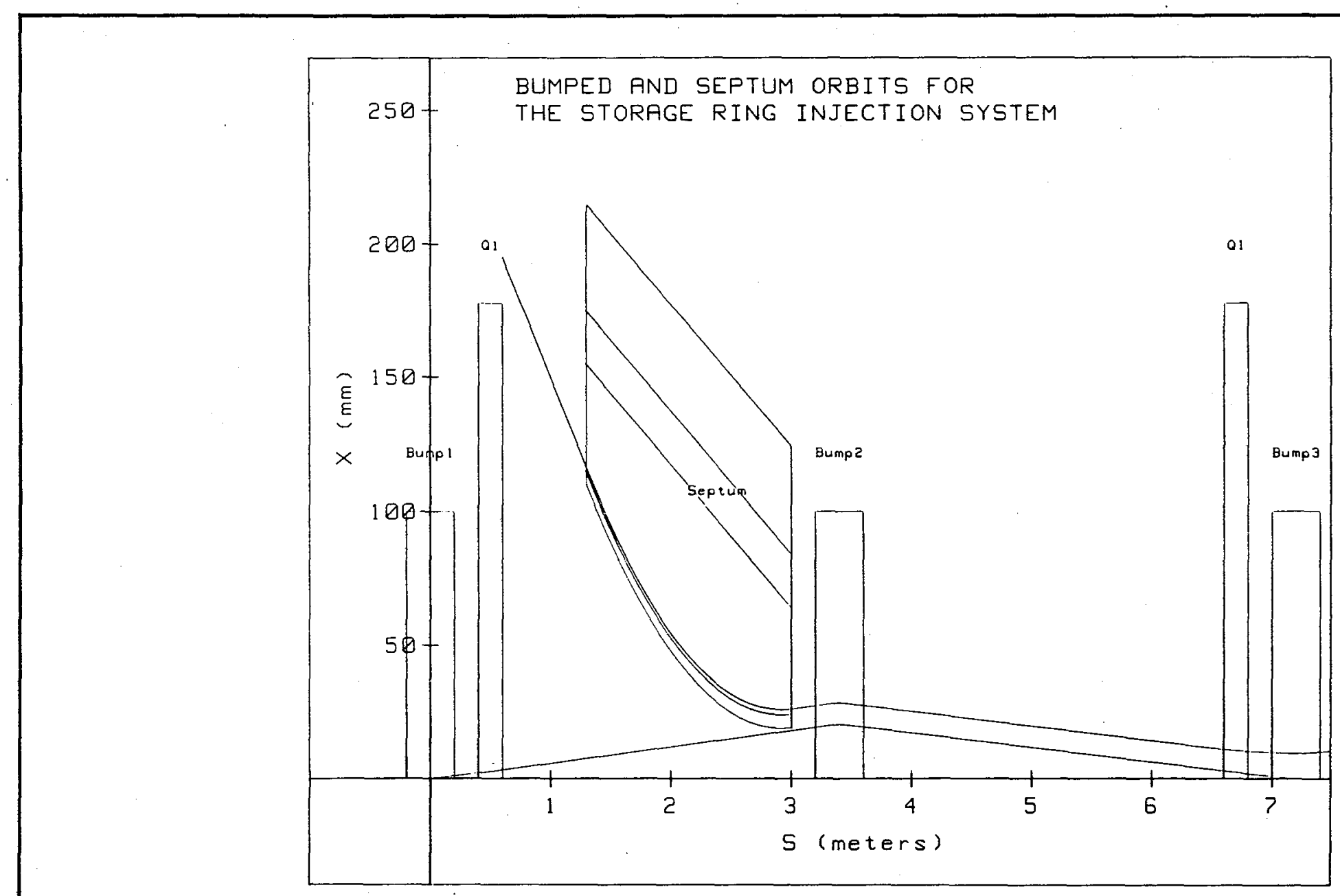

Figure 27. Storage ring injection system. 
be adjusted to accommodate the tune of the synchrotron ring. The pulsed magnets are fitted with ceramic beam tubes having a thin conductive metal coating. The conductive coating allows the pulsed magnetic field to penetrate the vacuum chamber without interrupting beam-image currents.

The magnetic septa for injection and extraction have conventional designs and operate continuously during the injection cycle. Coil ends are terminated in iron field clamps, and back-leg windings are incorporated in the design to minimize perturbations of the booster and storage-ring circulating beams.

\section{Linear Accelerator}

The electron linear accelerator is a $50-\mathrm{MeV}$ S-band linac about $8 \mathrm{~m}$ long. The electron beam from the linac is modulated, or chopped, to provide the required time structure to fill the rf buckets in the booster synchrotron. The use of different operating modes permits varying the number of electron bunches in the booster and in the storage ring. All the rf buckets can be filled with electron bunches (aside from a gap in the train of bunches caused by the injection kicker magnet in the booster), some of the buckets can be filled, or even a single bucket can be filled in the booster and storage ring. To fill all the buckets, a 185-ns, 20-mA linac beam pulse fills 80 percent of the booster circumference, leaving a 40-ns gap for the rise time of the extraction kicker magnet. Fast, single-turn injection is used in this mode. An alternative mode of linac operation is required when the storage ring is to be filled with only a single bunch of electrons, to accommodate experiments that require a long "dead time" between photon pulses. For the single-bunch or few-bunch modes of operation, the linac beam is modulated so that each burst consists of a train of 2-ns pulses at intervals of $32 \mathrm{~ns}$. Multi-turn injection into the booster puts all of these pulses into seven equally spaced rf buckets in the booster.

The linac can deliver up to $50 \mathrm{~mA}$, with a small duty factor. The major beam components of the linac include a chopped electron gun, an S-band buncher phase-locked to the linac, and two sections of standard corrugated-wave-guide (CWG) accelerator. Other major linac components include a 30-MW klystron amplifier and power system, a signal source, and an rf control system.

The electron gun has the capability of producing a pulse of $100-\mathrm{keV}$ electrons with a current of $1 \mathrm{~A}$. The entire electron-gun assembly has a standard commercial design, and it can be readily purchased. A pulser is mounted inside the high-voltage gun structure and is dc coupled so that it can produce either a single 2-ns pulse for single-bunch operation or a pulse train to fill all the rf buckets. The buncher operates at $2856 \mathrm{MHz}$ and is phase-locked to the injector linac. Since only about $1 \mathrm{~kW}$ is required, its power is taken from a coupler on the linac input-power transmission line (see Figure 28).

The traveling-wave linac consists of two sections of constant-gradient CWG accelerator. We estimate the linac emittance at about $6.4 \times 10^{-6} \pi \mathrm{m}$-rad in each transverse plane.

The very low duty factor in the pre-injector limits the average rf power dissipation in the accelerator components to less than $500 \mathrm{~W}$. A heated-water system operating at $113^{\circ} \mathrm{F}$ maintains the tuning stability in the CWG sections.

A single commercially available klystron, rated at over $30 \mathrm{MW}$, provides ample power for all linac rf accelerating structures. The klystron output power is split into two equal parts with a hybrid junction to power the two CWG sections. The outputs of the CWG 


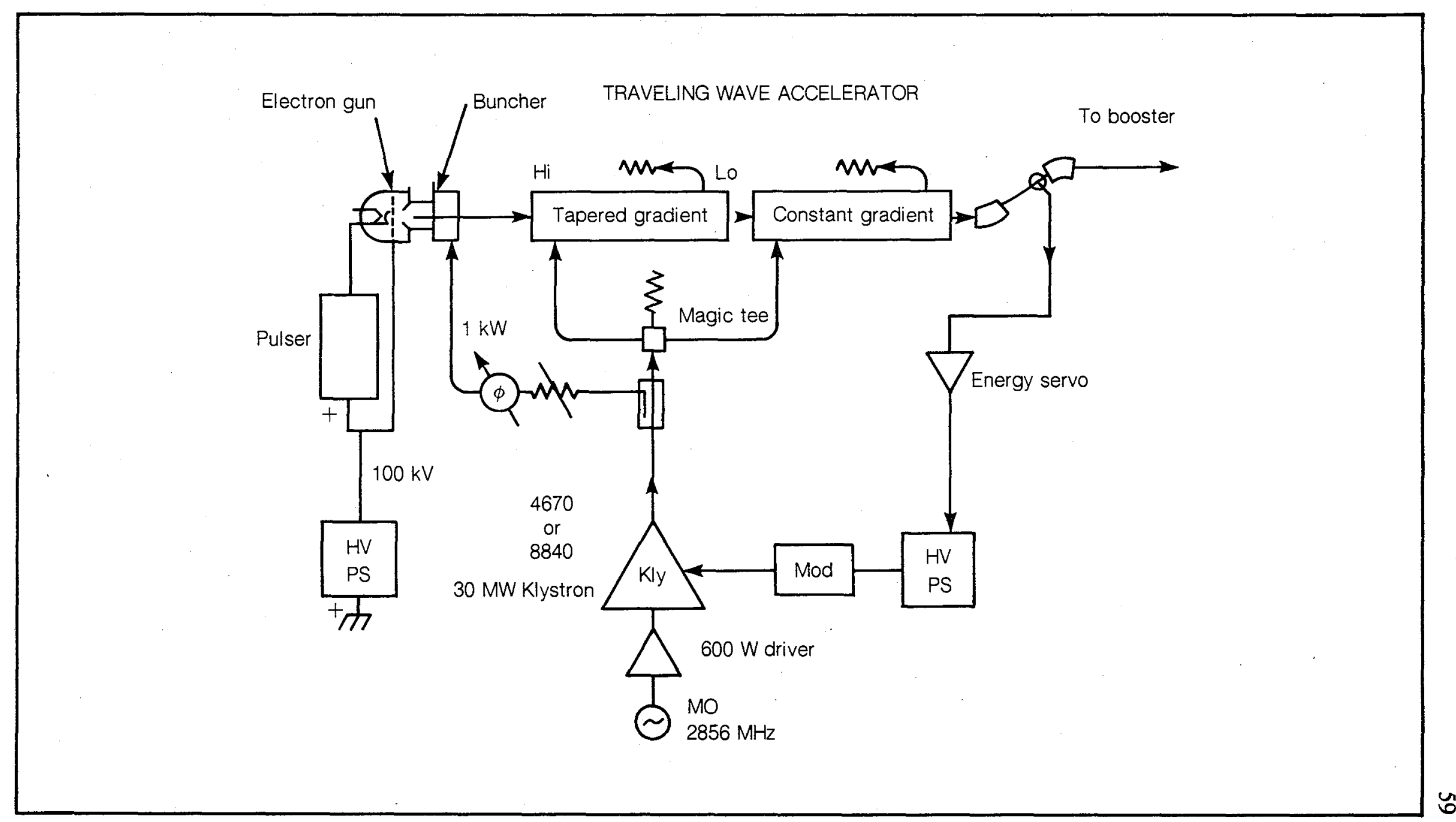

Figure 28. Linac RF system. 
sections terminate in tapered resistive waveguides. Heated water provides the temperature stability required for the phase stability of all transmission line components. A single window isolates the klystron vacuum system from all waveguide power-transmission components, which will operate within the linac vacuum.

The main klystron amplifier is powered by a standard line-type modulator of wellproven design. The modulator output drives the primary of a 1-to-10 step-up pulse transformer mounted directly on the tube base. This provides the required beam power of $250 \mathrm{kV}$ at $250 \mathrm{~A}$. The pulse-forming network (PFN) is tuned to a rising output voltage as a first-order compensation for beam loading in the linac. The PFN is designed for a pulse length of about three to five microseconds, to accommodate multi-turn injection. The modulator high-voltage supply is regulated with a signal from an energy monitor in the injection line. This keeps the output energy constant, even if the tuning shifts slightly with time or temperature.

The drive chain consists of a small $1-\mathrm{kW}$ klystron and a stable $2856-\mathrm{MHz}$ signal source, along with several monitoring and control components. The control system provides a gentle turn-on after a fault trip to prevent excessive window and waveguide arcing.

\section{Positron Injection Option}

In most electron storage rings the trapping of positive ions produced by the ionization of the residual gas can lead to increased emittance or to shortened beam lifetime. This issue was discussed earlier in the sub-section on "Major Parameters" of the storage ring. It was shown that these problems can probably be avoided at the ALS, because it is likely that the ALS storage ring can be operated in modes in which positive ions are driven to the wall in a time much shorter than the time for neutralization (about 2 seconds at $10^{-9}$ torr).

In the event that these techniques for avoiding ions are unsuccessful, an alternative approach is to replace the electron beam in the storage ring with positrons. The positive ions are then repelled by the stored beam and, hence, do not present any problem. The positron-beam approach is certain to avoid the positive-ion trapping problem, but it is more complicated than the straightforward electron-beam approach.

Although it appears quite likely that the ALS can be operated successfully with an electron beam, it is not possible at this time to present a definitive argument that positrons will not be required for the ALS to reach all of its performance goals, especially the very small design emittance of the electron beam. It therefore appears expedient to hold open the option of a positron injection system for the present time. However, we anticipate that our ability to predict the necessity for positrons will improve substantially in the coming months. First, operating experience with new storage rings will undoubtedly produce data which will be illuminating regarding these issues. Among such storage rings are Aladdin, the NSLS X-ray Ring, the SRS at Daresbury, the KEK Photon Factory, and the damping rings for the SLAC Linear Collider. Second, we plan to design and to help conduct experiments on existing storage rings, in order to understand better the phenomena of ion trapping and emittance growth. Last, the calculations and simulations discussed earlier will be continued and expanded. To conclude, we believe that the understanding of ion trapping can be developed to the point where a very rational decision can be made concerning the positron injection option for the ALS. This decision must be made approximately one year after start of construction funding. This will leave three years for positron injection system construction before the completion of storage ring construction. The rest of this Section 
describes a positron injection option and presents the estimated cost in terms of FY83 dollars.

The positron source uses a linac with a folded recirculating configuration in which most of the structure is used once for the acceleration of electrons to the positron converter, and then once again for acceleration of the positrons. This is shown in Figure 29. This positron beam from the linac is fed to the booster and thence to the storage ring.

The electron gun injects electrons into the first section of a two-section linac, and the intermediate-energy electrons are brought around a 180-degree bend to be accelerated further by a second, longer section of accelerator. They are then deflected another 180 degrees and brought to the positron radiator. The positrons are collected in a tapered solenoid and accelerated in a special $50-\mathrm{MeV}$ section in which additional focusing in the form of solenoids and quadrupoles provides the capability to accept the large transverse emittance of the positron beam.

The $50-\mathrm{MeV}$ positron beam is then re-injected into the two sections of linac that had accelerated the original electrons. A simple trio of magnets combines the $50-\mathrm{MeV}$ positron beam and the $100 \mathrm{kV}$ beam from the electron gun. At the 180-degree bend, small magnets separate the trajectories of the positrons and electrons, which have their own 180-degree transport system. The positrons are further accelerated in the second long section of linac and are split off by another trajectory separation magnet.

The parameters of the positron injection option are described in Table 13. 


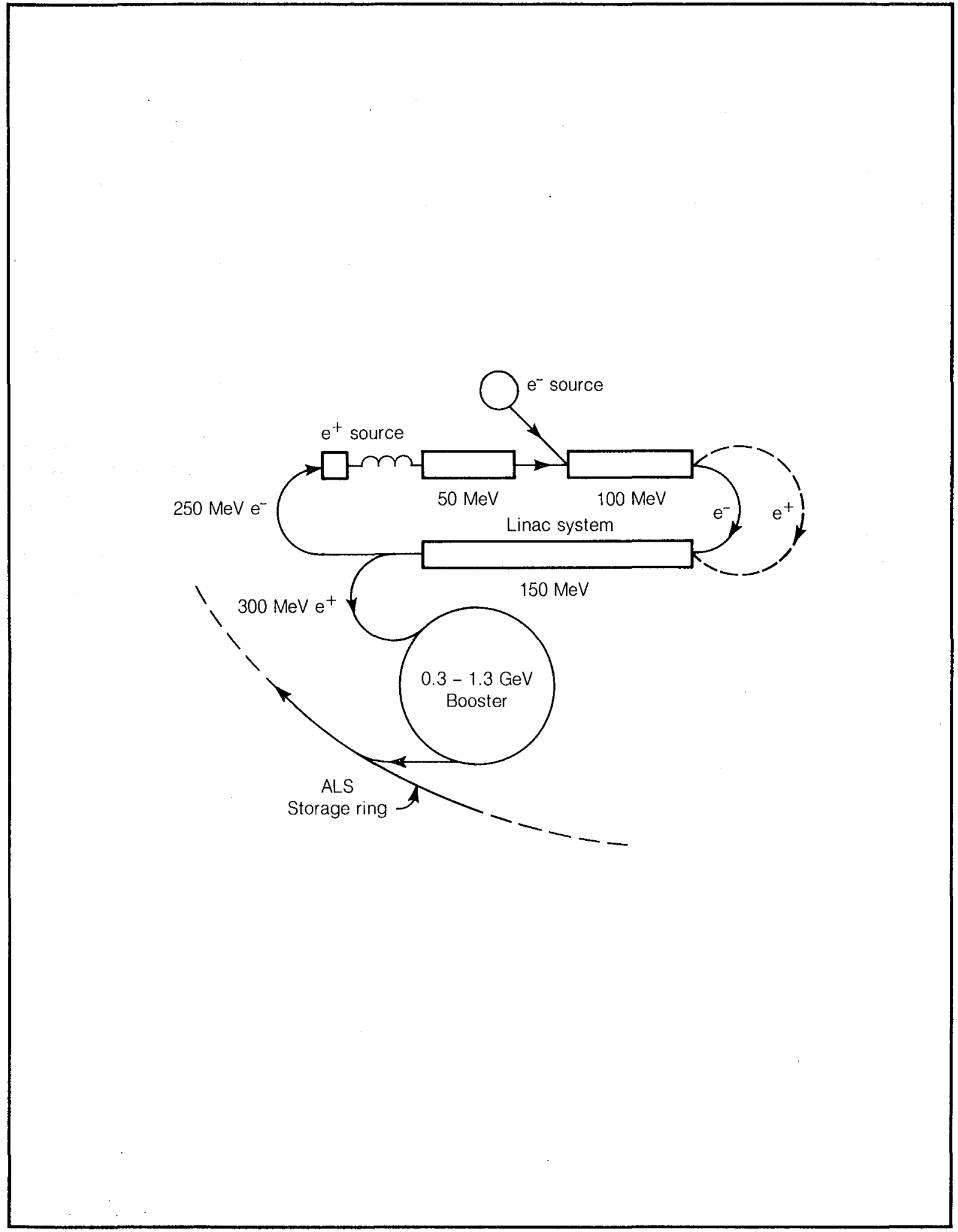

Figure 29. Positron injector option.

XBL $833-8925$ 
Table 13

Positron Injection Option

\begin{tabular}{ll}
\hline Injector & $100 \mathrm{kV}, .25 \mathrm{~A}$ \\
Pulse length & $700 \mathrm{~ns}, 3$ booster turns \\
First section & $100 \mathrm{MeV}, 8 \mathrm{MeV} / \mathrm{m}$ \\
$\quad$ Length & $12.5 \mathrm{~m}$ \\
Second section & $150 \mathrm{MeV}, 8 \mathrm{MeV} / \mathrm{m}$ \\
$\quad$ Length & $19 \mathrm{~m}$ \\
$\mathrm{e}^{-}$energy on radiator & $250 \mathrm{MeV}$ \\
$\mathrm{e}^{-}$spot radius & $2 \mathrm{~mm}$ \\
$\mathrm{e}^{+}$conversion efficiency & $0.31 \%$ \\
$\mathrm{e}^{+}$accepted momentum & $6-11 \mathrm{MeV} / \mathrm{c}$ \\
$\mathrm{e}^{+}$transverse momentum & $.83 \mathrm{MeV} / \mathrm{c}$ \\
$\mathrm{e}^{+}$charge/pulse & $3.6 \times 10^{9} / \mathrm{pulse}$ \\
Third (special) section & $50 \mathrm{MeV}, 8 \mathrm{MeV} / \mathrm{m}$ \\
$\quad$ Length & $6.3 \mathrm{~m}$ \\
Repetition rate & $2 \mathrm{~Hz}$ \\
$\mathrm{e}^{+}$flux & $7.2 \times 10^{9} \mathrm{e}^{+} / \mathrm{sec}$ \\
$\mathrm{e}^{+}$energy & $300 \mathrm{MeV}$ \\
transverse emittance & $4.9 \pi \mathrm{mm}-\mathrm{mrad}$ \\
Total linac length & $38 \mathrm{~m}$ \\
Total rf power & $100 \mathrm{MW}$ \\
\hline
\end{tabular}

The conversion efficiency calculation comes from the SLAC Blue Book and is consistent with several operating positron sources. In this design, the positron converter operates much as does the SLAC converter, with a momentum acceptance from 6 to 11 $\mathrm{MeV} / \mathrm{c}$ and a transverse acceptance of $.83 \mathrm{MeV} / \mathrm{c}$. The spot size of $2 \mathrm{~mm}$ represents a lower thermal load on the positron production target than is true for the SLAC device, because of the much lower incident electron energy.

The special $50-\mathrm{MeV}$ section that follows the converter and tapered solenoid is immersed in a focusing solenoid for part of its length, and is constructed in sub-sections to allow the insertion of frequent quadrupole doublets. By the end of the section, the focusing requirements are sufficiently reduced so that the positron beam can share a conventional section of linac with the electron beam.

A further area of optimization and cost savings involves the linac gradient and rf power requirement. The costs are minimized in the very high gradient regime. However, few linacs have actually been built with gradients of $20 \mathrm{MeV} / \mathrm{m}$ or greater, although one is being considered for the LEP injector. There is probably little risk in this venture, but for a linac as short as this one, the cost benefit may be marginal. This remains an area open for investigation.

In the positron-injection system shown in Figure 29, the 300-MeV, 0.7- $\mu \mathrm{sec}$ positron beam pulse is fed into the booster by the three-turn method of injection. After acceleration 
to $1.3 \mathrm{GeV}$ in the booster, the 92 useful positron bunches are transferred to the storage ring, just as in the many-bunch electron-beam mode of operation. With the booster cycling at $2 \mathrm{~Hz}$, the filling time to $400-\mathrm{mA}$ circulating beam in the storage ring is about 16 minutes, assuming 50-percent injection and transfer efficiency both in the booster and in the storage ring.

This positron injection system can also fill the storage ring for operation in the fewbunch modes. However, it can inject beam quickly only for low-current operation.

The estimated incremental cost of the positron-injection system is given in Table 14. The $50-\mathrm{MeV}$ linac used in the electron-injection system is assumed to be used for positron injection as well.

Table 14

Positron Injection Incremental Costs

(millions of FY83 dollars)

\begin{tabular}{ll}
\hline Linac & 4.0 \\
Positron converter system & 0.4 \\
Beam transport and & \\
$\quad$ extra linac focusing & 1.1 \\
$2 \mathrm{~Hz}$ booster modification & 0.5 \\
$\quad$ Totals & 6.0 \\
\hline
\end{tabular}

The linac costs are based on the cost analysis used for the $50-\mathrm{MeV}$ linac of the electron-injection system. The cost of the positron converter is based on the SLAC estimate for the positron converter in the Stanford Linear Collider Conceptual Design Report [13].

\section{Control System}

The ALS has a modern control system based upon the use of a system of microprocessors to provide distributed intelligence. This design strategy was successfully pioneered at LBL on the Uranium Beams Project. The recent advent of the microprocessor has led to an explosive improvement in computer technology, so that it is now possible to build a highly decentralized accelerator control system that provides exceptional performance at a reasonable price. The current trend is clear indeed: the Positron-Electron Project (PEP) uses a few computers, the Uranium Beams Project uses about 25, and the SLAC Linear Collider (SLC) will use about 100. For the ALS, we plan to use 100 to 200. The major benefits of such a decentralized architecture are a tremendous improvement in system response, an ability to monitor far more variables, and a substantial simplification of programming effort. A basic strategy used successfully for the Uranium Beams Project is to use computer hardware resources to reduce programming costs wherever possible.

Figure 30 shows the ALS control system architecture, which will be described from the top down. The highest level of the control system consists of the industry-standard network Ethernet (a trademark of XEROX Corporation). This network uses commercially 
available hardware to provide communications among the following components of the control system: the Display Micro Modules (DMM's), which drive the accelerator operator consoles; disk storage systems; and several work stations. Disk storage systems are used to record accelerator data, to log errors, to hold data to be printed, and to perform other similar functions. The workstations are used for "number crunching" problems (such as accelerator closed-orbit calculations), which typically require only a modest amount of measured data from the rest of the control system. Work stations are also used for program development and for the diagnosis of problems after beam turn-on. One further function of the network is to provide controlled access by the experimenters to a very few accelerator parameters.

Operating consoles (two are planned) provide the human interface to the control system and to the accelerator, and these consoles are controlled by the DMM's. A very fast control-system response time (about 0.1 second) is highly desirable to avoid objectionable operator delays. To provide this quick response, the DMM incorporates a great deal of computing power in the form of multiple microcomputers, each dedicated to its own tasks. These computers operate in parallel and communicate with one another via MULTIBUS (a trademark of Intel Corporation). We note that a tentative choice of four Intel iAPX-286 microprocessor boards for each DMM provides performance exceeding that of a DEC VAX $11 / 780$ for a very modest price. Each DMM provides operator interface functions, drives the menus, and monitors accelerator problems. The operator console includes six color-TV monitors with touch panels and three knob panels with four knobs each. A substantial programming effort is required to produce the software for the DMM's, because of the complexity of both the calculations and the displays. However, key portions of the DMM software have already been written for the LBL Uranium Beams Project control system.

For the DMM to perform its assigned tasks, it must have access to a complete set of data that describes the current status of the various accelerator systems. These data are stored in the Collector Micro Module (CMM), which provides storage for accelerator data and responds to DMM requests for these data. In addition, the CMM communicates with the Input/Output Micro Modules (IOMM's) and Intelligent Local Controllers (ILC's), which provide the interface to accelerator hardware. This communication is provided by a number of serial links that together can provide the speed required for a quick controlsystem response time.

The ILC's and IOMM's are local computers (i.e., located near the accelerator) that ensure the proper operation of the accelerator hardware. They are directly interfaced to the accelerator components, and they are provided with considerable computing power (comparable to a 16-bit minicomputer) to perform the following functions: monitoring interlocks, data taking, closed-loop control, data analysis, and error checking. Only analyzed data are sent to the CMM, for the use of the DMM, so the latter is not bogged down with low-level control tasks. The ILC's and IOMM's differ primarily in size and capacity. The ILC's control a few devices with up to about 50 channels of input and output data. The IOMM's, however, are MULTIBUS card cages with up to 16 slots, and they control many devices with up to 250 channels of data. The most powerful IOMM's have multiple microcomputers operating in parallel to perform complex tasks requiring fast response times. A portable console that can interface directly to the ILC/IOMM will be provided for local control or debugging of the ILC's or IOMM's. 


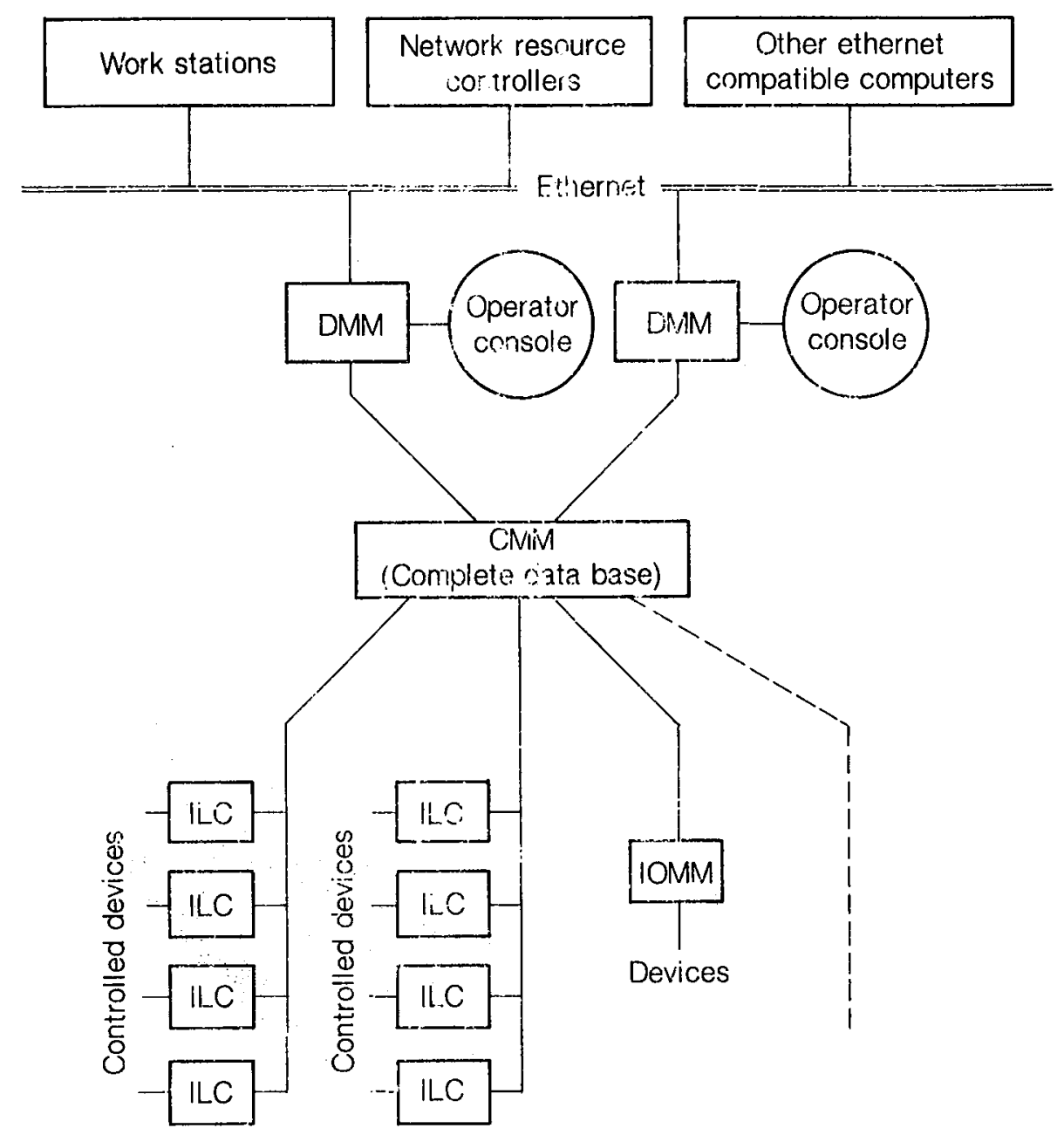

Figure 30. Accelerator control system. 
In many instances, there are advantages to using several ILC's rather than one IOMM. First, since each ILC deals with only one device, it is easy to provide excess computing capacity. This excess capacity greatly simplifies the programming task, since there is little or no need for the programmer to deal with problems caused by speed limitations of computer hardware. Second, this excess computing speed permits some tasks to be shifted from the DMM, which is the most complex component of the control system, to the ILC, which is the simplest. Examples of such tasks are error checking and conversions of numbers into different representations (from engineering units into ASCII, for instance). The consequences of shifting these tasks are to simplify the DMM software and to reduce software development and maintenance costs. Each ILC is connected to the rest of the accelerator control system through a serial link, so it can easily be isolated from the system during debugging or maintenance.

The use of ILC's also provides considerable flexibility in modifying the control system architecture. Each ILC must constantly refresh about 125 bytes of data at the complete data base stored at the CMM. It is possible for ten ILC's connected to a single 180-kbaud serial link to refresh 1250 bytes of data in about 100 milliseconds. Should it prove necessary, this refresh rate can be increased by reducing the number of ILC's connected to each serial link. In contrast, a similar modification would be difficult to accomplish if IOMM's were used, because the minimum fixed cost of each IOMM chassis is too high for a large increase in the number of IOMM's to be attractive. We estimate that about 27 serial links would be required for 170 ILC's (17 serial links) and 10 IOMM's (10 serial links). Since these serial links operate entirely in parallel, the total system communications capacity is very substantial, almost $5 \mathrm{Mbaud}$. Note that this is a steady-state speed, not a peak value. If required, about 50 serial links could be used to provide a communications speed of 9 Mbaud.

Computer timing and synchronization are provided by a Manchester encoding system (used for the Uranium Beams Project) with a fiber-optics link. This system provides timing control to a few hundred microseconds.

\section{Insertion Devices}

The task of the accelerator design team is to provide an electron storage ring with characteristics (such as low emittance, short bunches, and long straight sections) that permit great flexibility in the selection of insertion devices (wigglers and undulators). The ALS storage ring described earlier meets these criteria. Altogether, 12 long straight sections are available, and 12 insertion devices can ultimately be accommodated. Initially, however, a complement of only six devices (four undulators and two wigglers) is planned.

We have selected a set of possible insertion devices, whose properties are summarized in Table 13. We recognize, however, that the users of the facility must be involved in the final choice of insertion devices, and we are leaving open the possibility of modifications to the present list. Details of the various devices are presented below.

\section{Room-Temperature Insertion Devices}

The parameters of the six planned insertion devices are listed in Table 15. These parameters are chosen to maximize the high spectral brilliance of the undulation radiation and also to cover the maximum range of photon energies. 
Table 15

Tentative ALS Insertion Devices $(1.3 \mathrm{GeV})$

\begin{tabular}{llccccc}
\hline Name & $\begin{array}{c}\text { Insertion Device } \\
\text { Type }\end{array}$ & $\begin{array}{c}\text { Peak Field } \\
(\mathrm{T})\end{array}$ & $\begin{array}{c}\text { Period } \\
(\mathrm{cm})\end{array}$ & $\begin{array}{c}\text { No. of } \\
\text { Periods }\end{array}$ & $\begin{array}{c}\text { Length } \\
(\mathrm{m})\end{array}$ & $\begin{array}{c}\mathrm{E} \\
(\mathrm{eV})\end{array}$ \\
\hline $\mathrm{U}_{\mathrm{A}}$ & $\begin{array}{c}\text { Permanent Magnet } \\
\text { Undulator }\end{array}$ & 0.39 & 16.7 & 30 & 5 & $5-600$ \\
$\mathrm{U}_{\mathrm{B}}$ & $\begin{array}{l}\text { Hybrid Undulator } \\
\mathrm{U}_{\mathrm{C}}\end{array}$ & 0.74 & 6.25 & 80 & 5 & $25-1500$ \\
$\mathrm{U}_{\mathrm{D}}$ & Hybrid Undulator & 0.54 & 5.0 & 100 & 5 & $75-3000$ \\
$\mathrm{~W}_{\mathrm{E}}$ & Hybrid Undulator & 0.57 & 3.5 & 142 & 5 & $\begin{array}{r}200-5000 \\
\mathrm{~W}_{\mathrm{F}}\end{array}$ \\
$\begin{array}{l}\text { Hybrid Wiggler } \\
\text { Superconducting } \\
\text { Wiggler }\end{array}$ & 1.60 & 10.0 & 25 & 2.5 & $\begin{array}{r}0.1-10000 \\
1-20000\end{array}$ \\
\hline
\end{tabular}

The high-field wiggler $\mathrm{W}_{\mathrm{F}}$ will, of course, use superconducting technology. This device will be described separately in the next Section. The low-field undulator $U_{A}$ has a field that is low enough to build it as a pure permanent magnet device. The other insertion devices $\left(U_{B}, U_{C}, U_{D}, W_{E}\right)$ will be built as hybrid devices using both samarium-cobalt permanent magnets and vanadium permendur pole pieces. Undulator $U_{D}$ and wiggler $W_{E}$ have the smallest poletip gaps, $1.2 \mathrm{~cm}$, at the indicated fields, which will result in a $1.0-\mathrm{cm}$ minimum beam aperture. These hybrid designs will be very similar to that of the wiggler for the LBL/Exxon beam line at SSRL, which is now close to completion. Undulator $\mathrm{U}_{\mathrm{A}}$ is similar to the undulator built by LBL for SSRL two years ago. The two SSRL devices are shown in Figures 31 and 32. Table 16 gives some operating parameters of the ALS insertion devices.

Table 16

Tentative ALS Insertion Devices Parameters, Openings Angles and Power Output (1.3 GeV, $400 \mathrm{~mA})$

\begin{tabular}{|c|c|c|c|c|c|c|c|c|}
\hline Name & $\begin{array}{c}\mathrm{K} \\
(\mathrm{T}-\mathrm{cm})\end{array}$ & $\begin{array}{l}\text { Magnet } \\
\text { Gap } \\
\text { (cm) }\end{array}$ & $\begin{array}{c}\text { Critical } \\
\text { Energy } \\
(\mathrm{keV})\end{array}$ & $\begin{array}{l}\text { Horizontal } \\
\text { Opening } \\
\text { Angle } \\
( \pm \mathrm{mrad})\end{array}$ & $\begin{array}{c}\text { Vertical } \\
\text { Opening } \\
\text { Angle } \\
( \pm \text { mrad })\end{array}$ & $\begin{array}{l}\text { Power } \\
\text { (watts) }\end{array}$ & $\begin{array}{c}\text { Peak Power } \\
\text { Density } \\
\text { (watts } / \mathrm{mrad}^{2} \text { ) }\end{array}$ & $\begin{array}{c}\text { Peak Power } \\
\text { Density } \\
@ 1.9 \mathrm{GeV} \text {, } \\
\text { (watts } / \mathrm{mrad}^{2} \text { ) }\end{array}$ \\
\hline $\mathrm{U}_{\mathbf{A}}$ & 6.04 & 1.43 & 0.44 & 2.37 & .392 & 321 & 143 & 653 \\
\hline $\mathrm{U}_{\mathbf{B}}$ & 4.31 & 1.91 & 0.83 & 1.69 & .392 & 1170 & 731 & 3336 \\
\hline $\mathrm{U}_{\mathrm{C}}$ & 2.53 & 1.75 & 0.61 & 0.993 & .392 & 624 & 666 & 3040 \\
\hline $\mathrm{U}_{\mathrm{D}}$ & 1.87 & 1.20 & 0.64 & .734 & .392 & 696 & 1001 & 4563 \\
\hline $\mathrm{W}_{E}$ & 14.94 & 1.20 & 1.80 & 5.89 & .392 & 2741 & 491 & 2240 \\
\hline$W_{F}$ & 65.66 & 3.50 & 5.62 & 25.71 & .392 & 21412 & 878 & 4009 \\
\hline
\end{tabular}




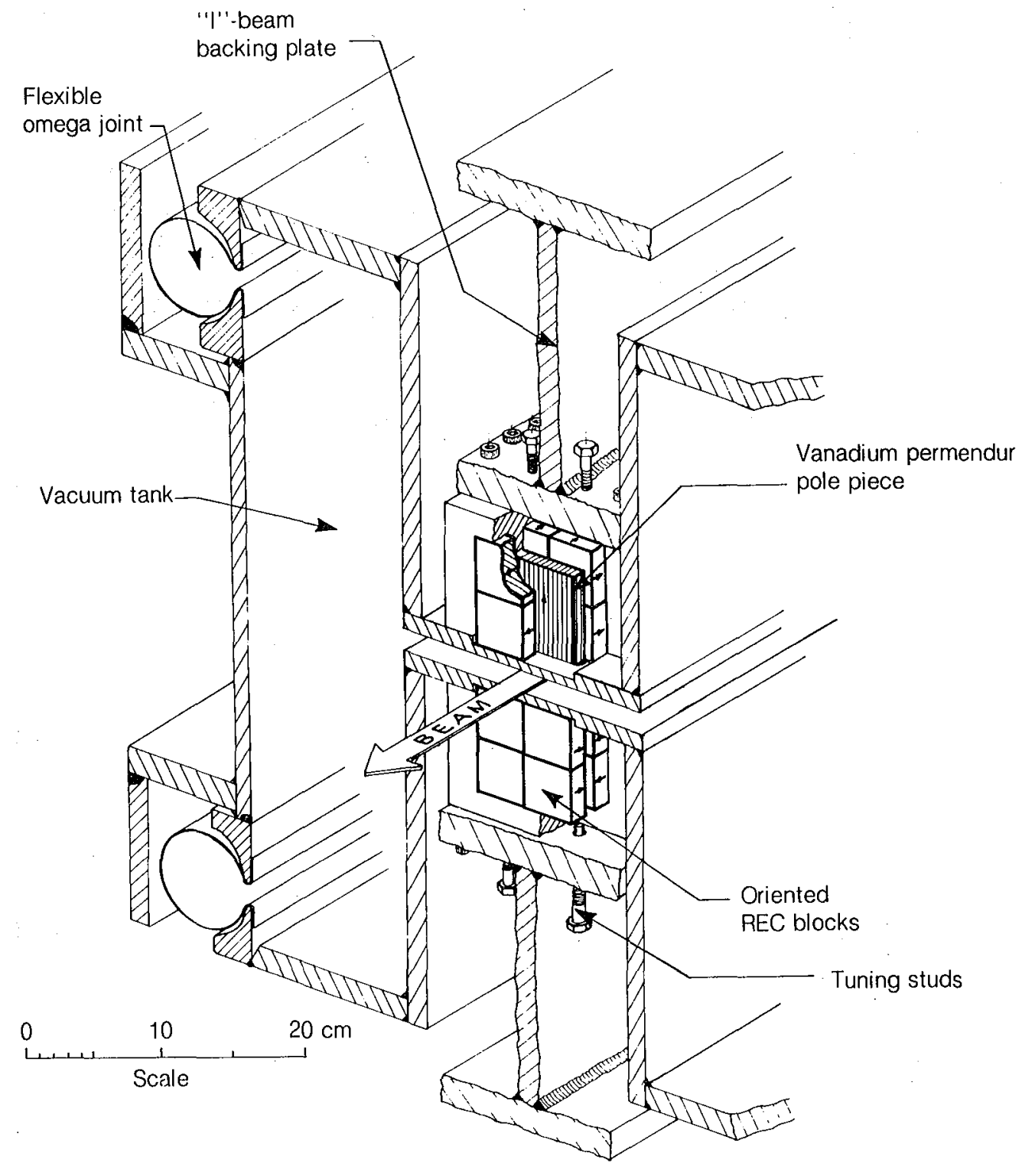

Figure 31. Beamline VI wiggler.

XBL 833-8953 


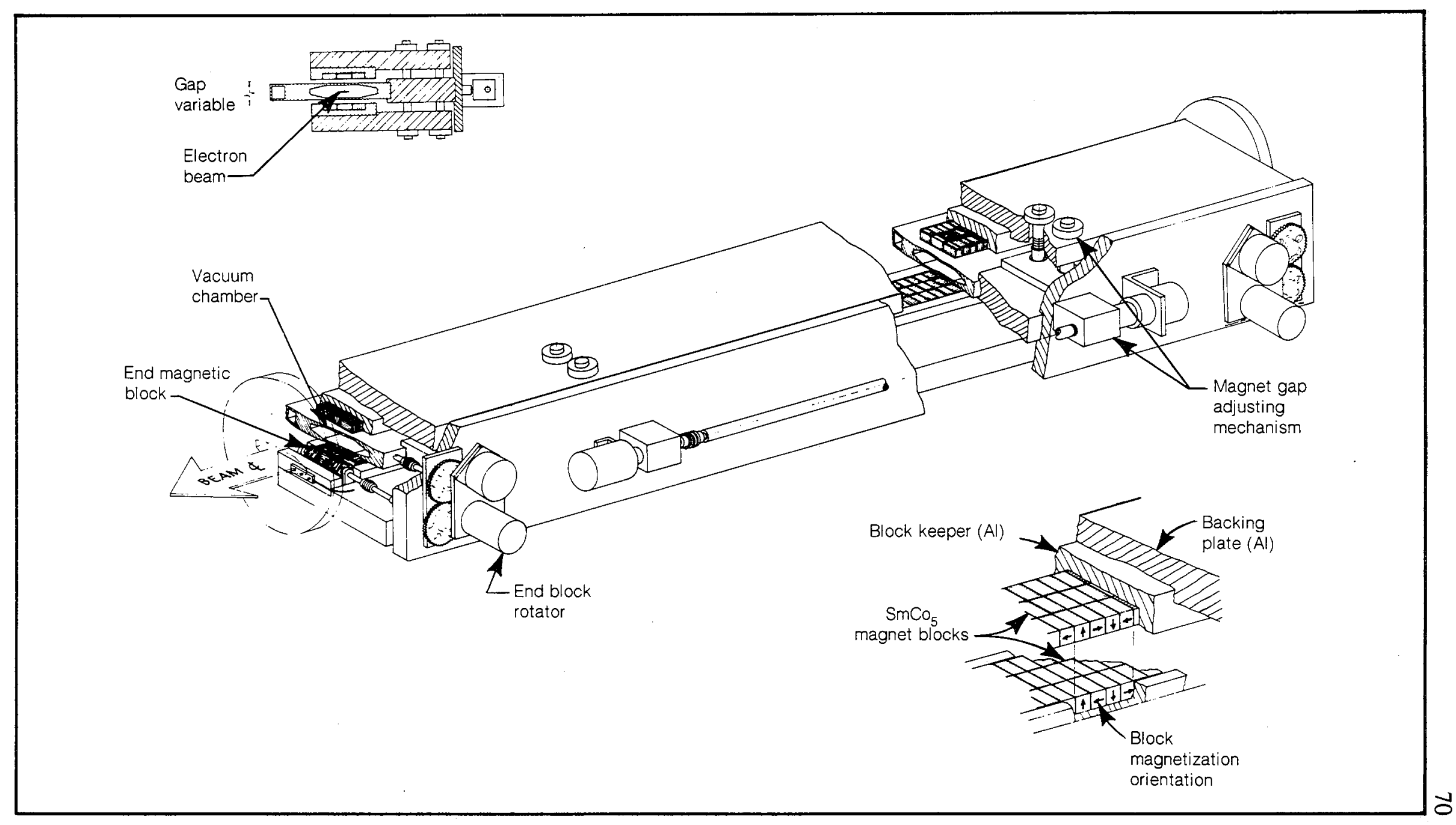

Figure 32. LBL-SSRL Undulator. 
The ALS lattice design, with low electron beam emittance and long straight sections, is ideal for good undulator performance. Figure 3 shows the extremely high photon spectral brilliance that the ALS undulators can provide. The range of photon energies available from undulators extends up to $10 \mathrm{keV}$ when the storage ring operates at $1.9 \mathrm{GeV}$ (or up to $40 \mathrm{keV}$ with a superconducting wiggler). The undulator spectrum consists of sharp peaks at discrete energy values, as shown in Figure 2. Scanning in energy during an experiment can be achieved by varying the gap size of the undulator to change the magnetic field.

The ALS wigglers provide attractively high photon fluxes for many types of experiments. A representative spectrum is shown in Figure 5.

\section{Superconducting Wiggler}

Parameters for the first ALS superconducting wiggler magnet have been chosen to supply synchrotron radiation for up to five beam lines for experimenters, with photon energies up to $40 \mathrm{keV}$. A design minimum of $5 \mathrm{~T}$ can be achieved with $4.5-\mathrm{K}$ operation; however, a cryogenic system with $2-\mathrm{K}$ capability is provided to allow operation up to $6 \mathrm{~T}$ and to accommodate a short-period, high-field design, if required in the future. The design of the magnet is conservative, using well-known conductor (ductile $\mathrm{Nb}-\mathrm{Ti}$ alloy) and the same type of 2-K cryogenic system that has been in service at the LBL high-field magnet test facility for two years. In addition, the magnet gap is large enough to allow the insertion of a liquid-nitrogen-cooled beam tube to shield the magnet from image-current heating and possible stray radiation. The fraction of distance along the beam line used for coil thickness (as opposed to pole length) has been optimized to minimize the amount of conductor required. The field distribution along the beam is slightly more sharply peaked than a sine wave. Poles and yoke of magnetic steel are used to control stray fields and to reduce the amount of superconductor required. An important additional advantage of the magnetic yoke is that the forces on the coils in the direction normal to the midplane are outward, rather than inward across the magnet gap. This means that no heavy structural elements are required in the gap, and thus the coils can be closer together and be more efficient. To provide pressure integrity of the liquid-helium vessel up to 5 atmospheres, stainless steel walls $5 \mathrm{~mm}$ thick are used to span the horizontal aperture. The poles at the entrance and exit are provided with trim coils so that the exit point and direction of the electron-beam orbits are not changed by the operation of the wiggler. Sensors and controls for the entire system are compatible with central-computer monitoring and steady-state control. If it were possible to decrease the magnet gap, by eliminating the liquid-nitrogen-cooled beam tube for example, it would be possible to increase the magnetic field strength or to reduce the period length. Parameters of the wiggler are listed in Table 17. 
Table 17

Superconducting Wiggler Parameters

Central Magnetic Field, variable with good field quality

Length of period (complete cycle of up and down field)

$\mathrm{K}$ value at $6 \mathrm{~T}$ 3 to $6 \mathrm{~T}$

Number of Periods 78

Overall Length

Aperture (full) $240 \mathrm{~cm}$

Pole Length in Beam Direction

Coil Thickness in Beam Direction

Conductor:

Material

Cross Section

$\mathrm{Cu}$ : Superconductor Ratio

Insulation

$14 \mathrm{~cm}$

Operating Temperature

$10 \mathrm{~cm}$ horiz. $\times 1 \mathrm{~cm}$ vert.

Operating Temperature

Magnet Poles and Yoke Material

Current Density in the Conductor

$1.5 \mathrm{~cm}$

$2.0 \mathrm{~cm}$

$\mathrm{Nb}-\mathrm{Ti}$ alloy in $\mathrm{Cu}$ matrix, monolithic $1.26 \mathrm{~mm} \times 1.26 \mathrm{~mm}$ 1.5:1 (by volume)

Formvar

$2 \mathrm{~K}$ (for $6 \mathrm{~T}$ ) $4.5 \mathrm{~K}$ (for $5 \mathrm{~T}$ ) magnetic steel

Higher performance can be achieved by using two "grades" of wire, one for high maximum field and another for the low-field region of the coil.)

The construction of the wiggler coil is shown in Figure 33. Its 58 identical coils are in the shape of flat "racetracks." The conductors are square with rounded corners and are spaced apart at the rounded ends to decrease the maximum field magnitude in the coil. This will permit permeation by liquid helium throughout the coil. Pole pieces are made of mild steel. The load-carrying yoke pieces can be made of either a laminated steel or a 9percent-nickel steel, which is a good structural steel at low temperatures.

Each coil has 225 turns of $1.26-\mathrm{mm}$-square multifilamentary wire with a copper-tosuperconductor ratio of 1.5. Total stored energy is $1.1 \mathrm{MJ}$. Each coil is shunted with a protection resistor $(\sim 0.01 \Omega)$ with which it is in good thermal contact. A low-voltage ( $\sim 4$ V) power supply can charge the coils in about 20 minutes.

During accidental quenches, each coil is small enough to discharge safely, depositing its energy internally. Furthermore, the coils are sufficiently coupled inductively to cause a chain of successive quenches that, if rapid enough, could provide a safe discharge without any quench protection circuitry. However, for maximum safety the appearance of the additional voltage across a single normal coil $(\sim 10 \mathrm{~V})$ will trigger the opening of a switch 

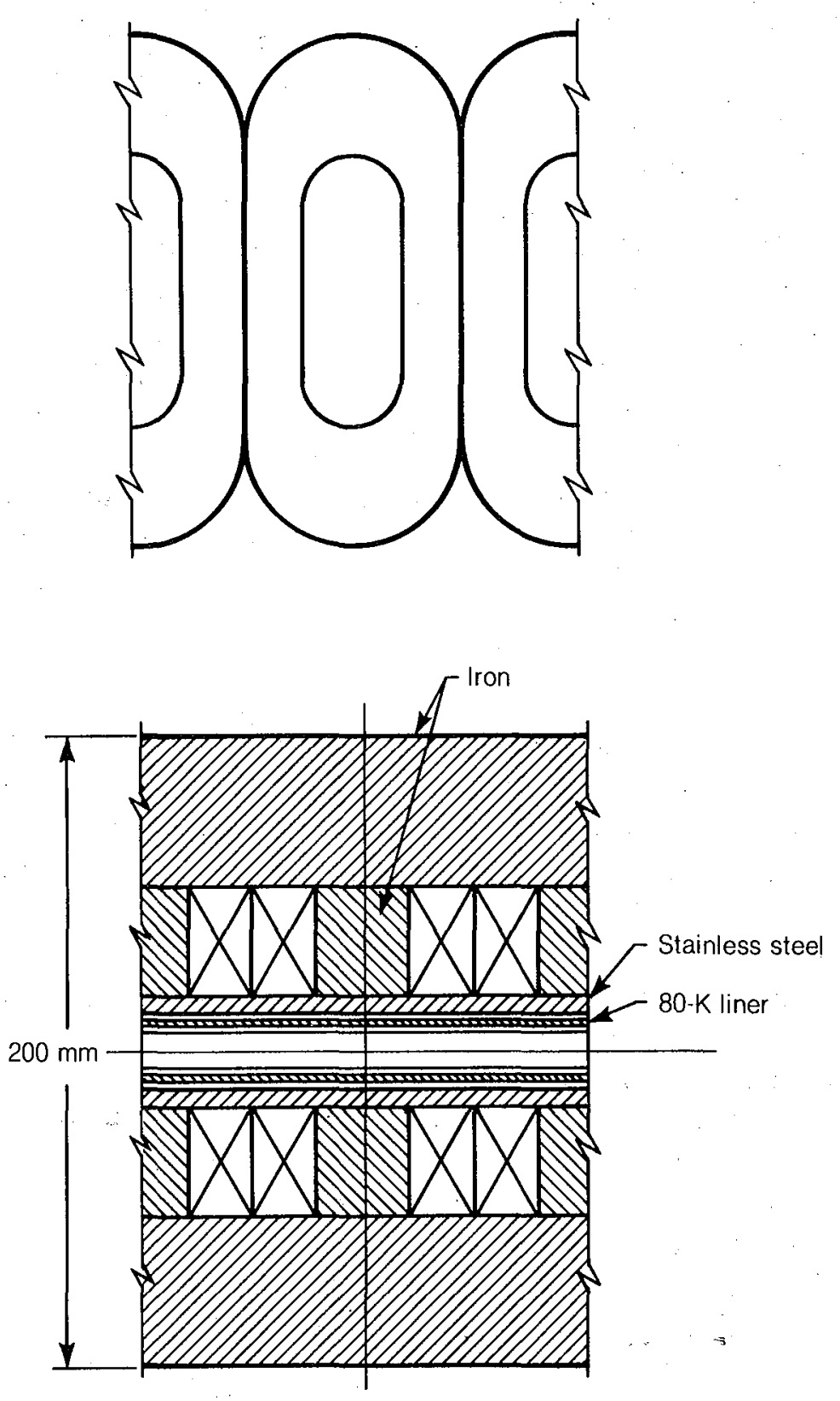

Figure 33. Superconducting wiggler magnet coil.

XBL 833-8955 
at the power supply. This will cause immediate transfer of current in each coil to its shunt resistor and will decrease the current in the shunt of the normal coil, thus limiting its heating. All coils will be quenched immediately because of heating in the shunts. This is a safe coil-protection system that allows high inductance, low current, and reasonable wire size. Alternative shunting methods, such as the cold diodes used in the FNAL Energy Doubler magnets and the BNL-CBA magnets, could also be used; this would eliminate the need for opening a switch for protection.

The cryogenic system, shown schematically in Figure 34, is nearly identical to the system used at the LBL high-field magnet test facility. The system produces helium II in a sub-cooled condition, at a pressure slightly greater than $1 \mathrm{~atm}$, to act as the magnet coolant. The heat leak to the system is calculated to be $70 \mathrm{~W}$ for $2-\mathrm{K}$ operation. A suitable liquifier for this service is the Model 1460 (Koch Process Systems, Inc.), a competitively priced commercial unit.

Figures 35 and 36 show the helium II cryostat closely coupled to a magnet dewar. Depending on space or shielding conditions, the cryostat can also be several meters from the magnet. A special transfer line can be used to carry the coolant, the current leads, and the instrument leads.

\section{Photon Beam Lines}

To use the synchrotron radiation efficiently, multiple photon beam lines can be built to use the radiation from a single insertion device. In some cases, the simultaneous operation of multiple beam lines is possible, but in other cases a removable mirror is used to switch the radiation from one beam line to another. Present plans call for fourteen photon beam lines to be constructed (see Figures 37 to 43) during the startup phase of the ALS; these beam lines will make use of the radiation from the initial complement of six insertion devices. These 14 beam lines have been planned in such a way that an additional 5 photon beam lines can be built at these 6 straight sections. Of course, we recognize that final plans for these experimental areas must be made in consultation with the users of the facility; thus, modifications to the layouts described here will be accommodated as required.

The first optical element of a beam line is usually a mirror. The glancing angle of incidence on the mirror must meet three criteria. First, the cutoff energy must lie above the desired range of the deflected beam line. Second, the thermal loading on the mirror must be within tolerable limits of present technology, i.e., less than about $10 \mathrm{~W} / \mathrm{cm}^{2}$. Third, enough working space must be provided between the beam lines. The first two requirements tend to make the glancing angle smaller, whereas the third makes it larger. Deflection angles of the ALS beam lines are obtained by balancing the above conflicting requirements.

Undulator radiation achieves its maximum spectral brilliance when observed through a pinhole, which for ALS is a hole $0.6 \mathrm{~mm}$ in diameter at $20 \mathrm{~m}$. Therefore, an undulator beam line is provided with a downstream slit assembly. If the main beam line has a secondary line, this means that the deflected and undeflected lines cannot be operated simultaneously. However, efficient use of the undulator line by several experimental groups can be achieved by producing separate monochromators for the main line and the secondary line. One team can then be performing an experiment in the main line while the other team is installing an experiment in the secondary line. 

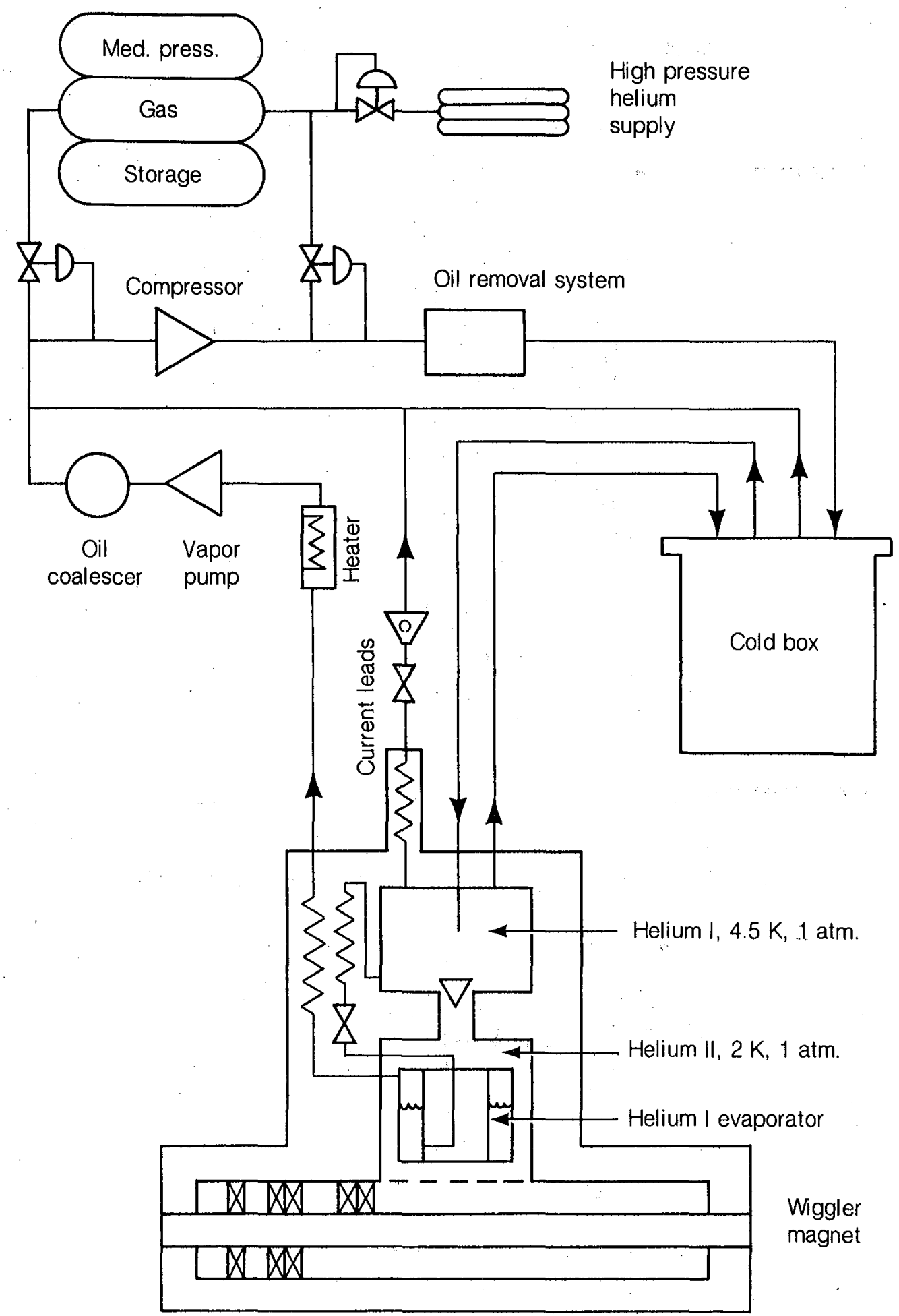

Wiggler

magnet

Figure 34. Schematic of 2-K helium refrigerator system.

XBL 833-8956 


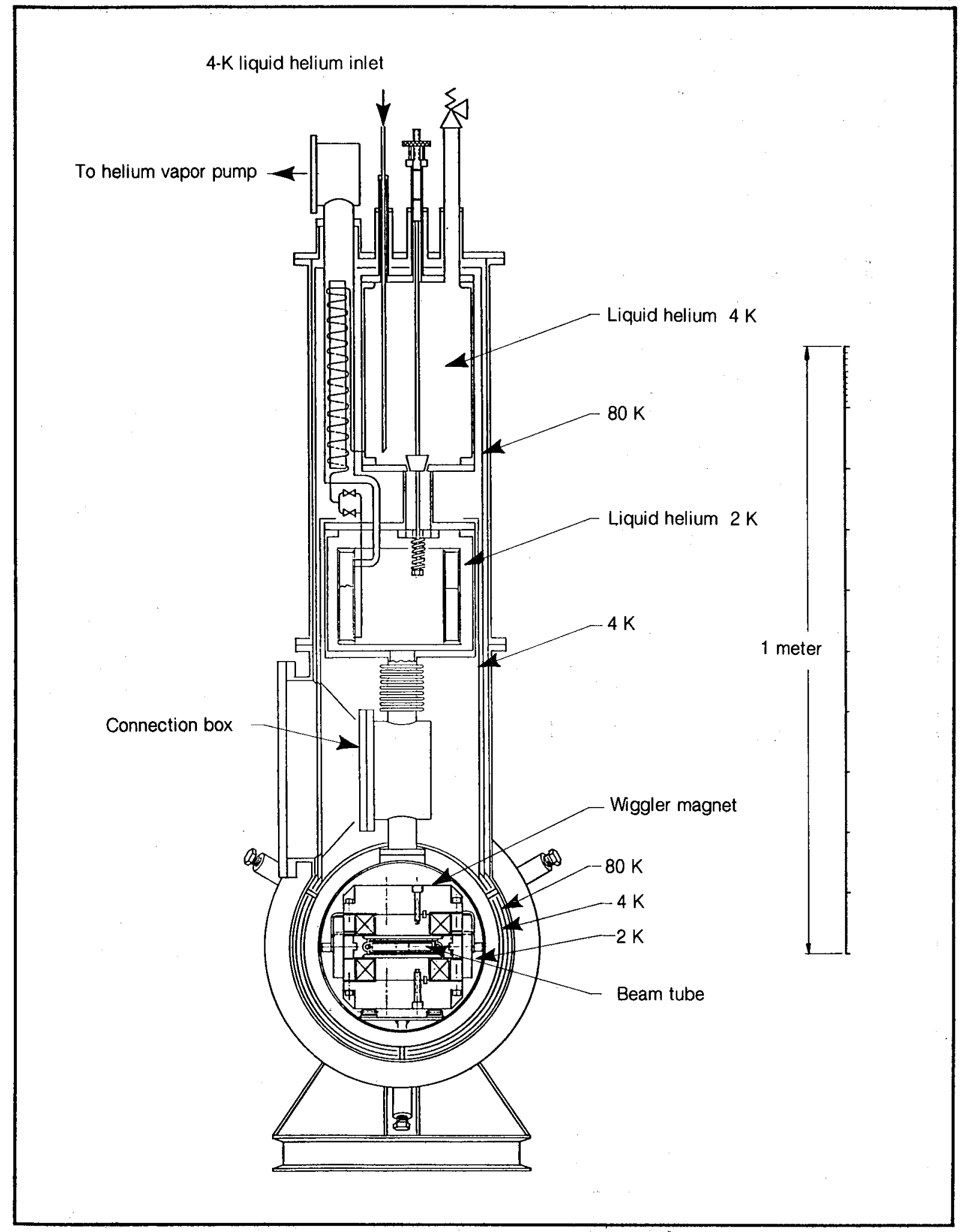

Figure 35. Superconducting wiggler cryostat. 


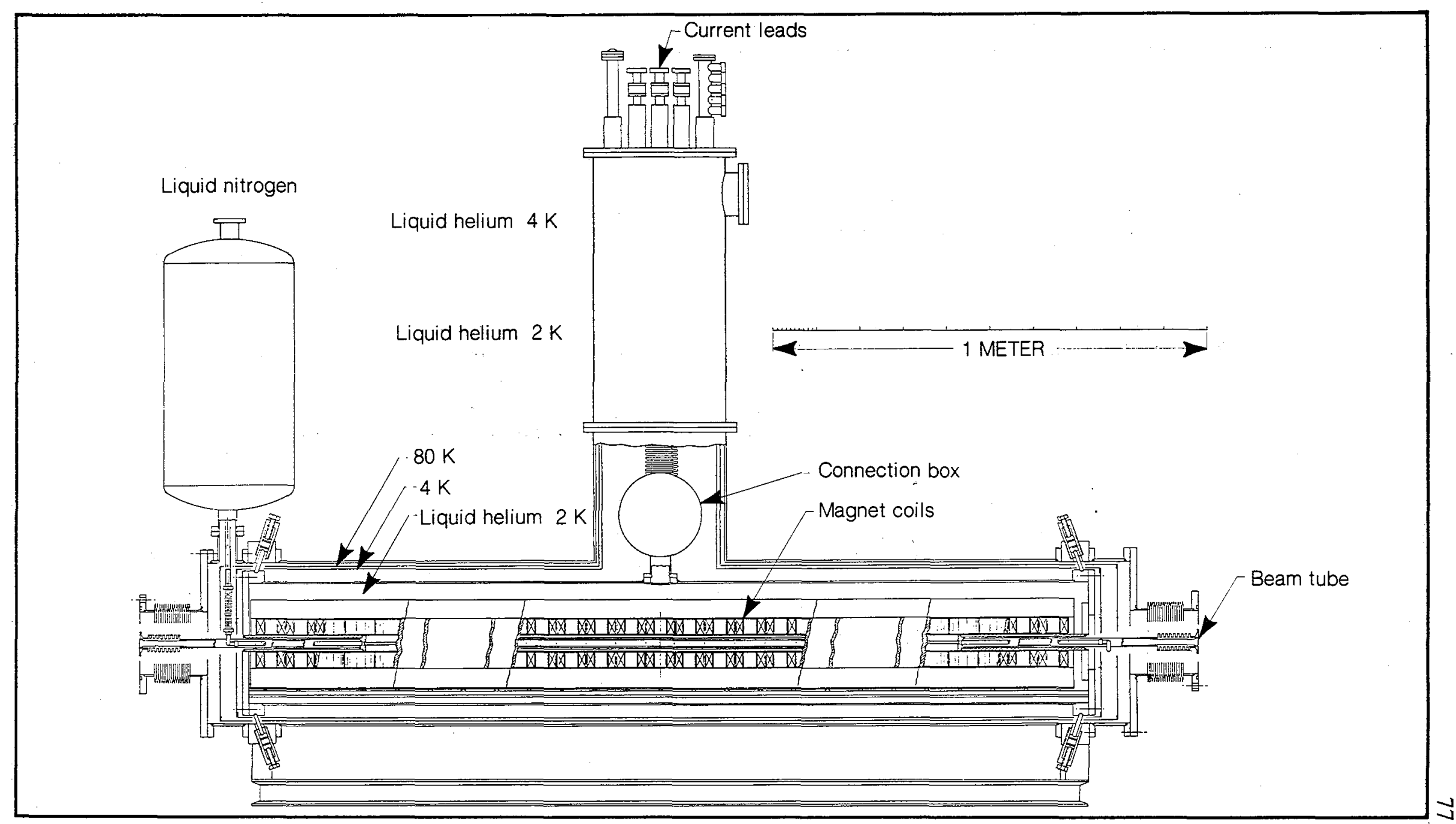

Figure 36. Superconducting wiggler cryostat. 


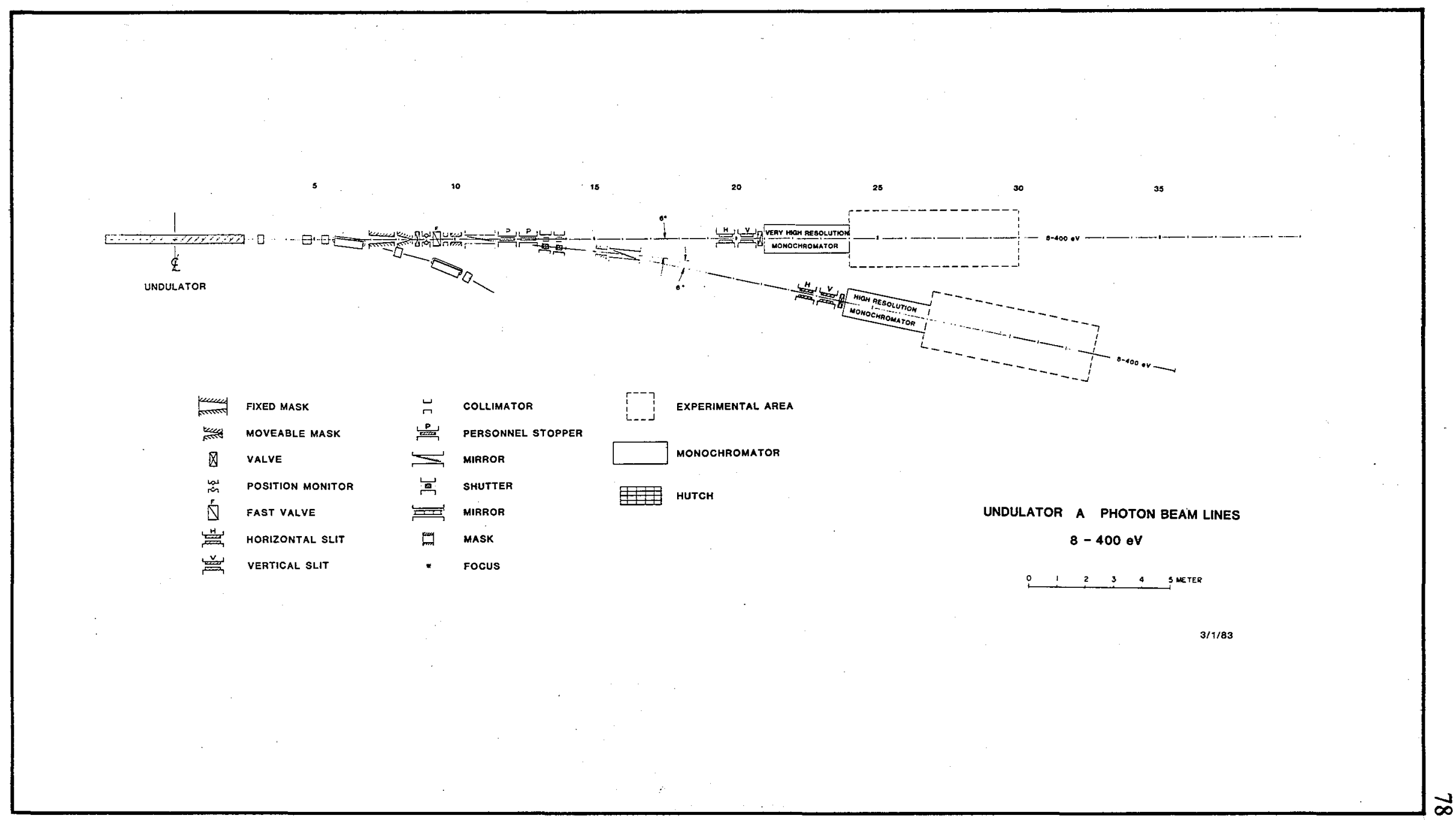

Figure 37. Undulator A photon beamlines.

XBL 8211-3488A 


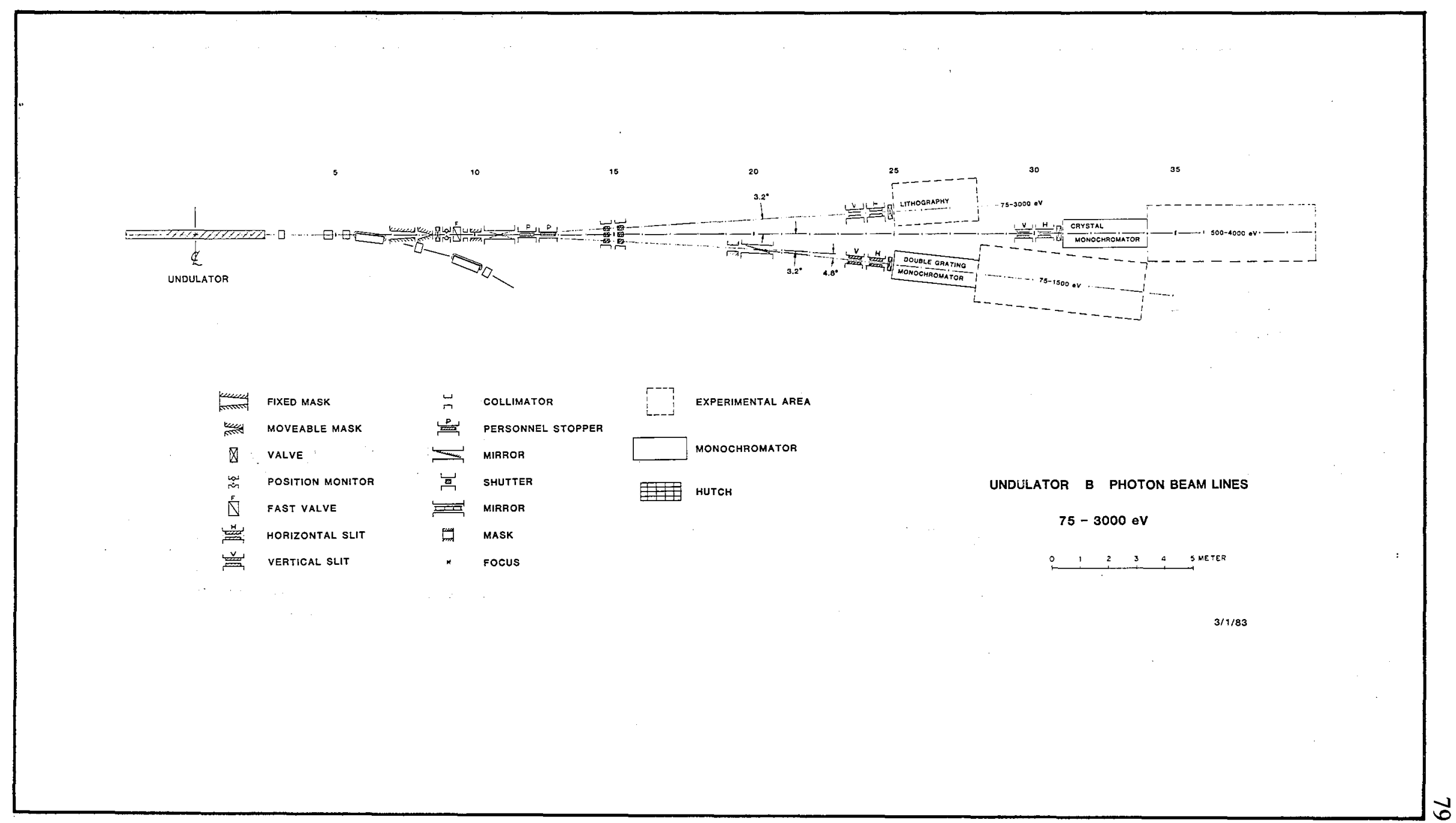

Figure 38. Undulator B photon beamlines. 


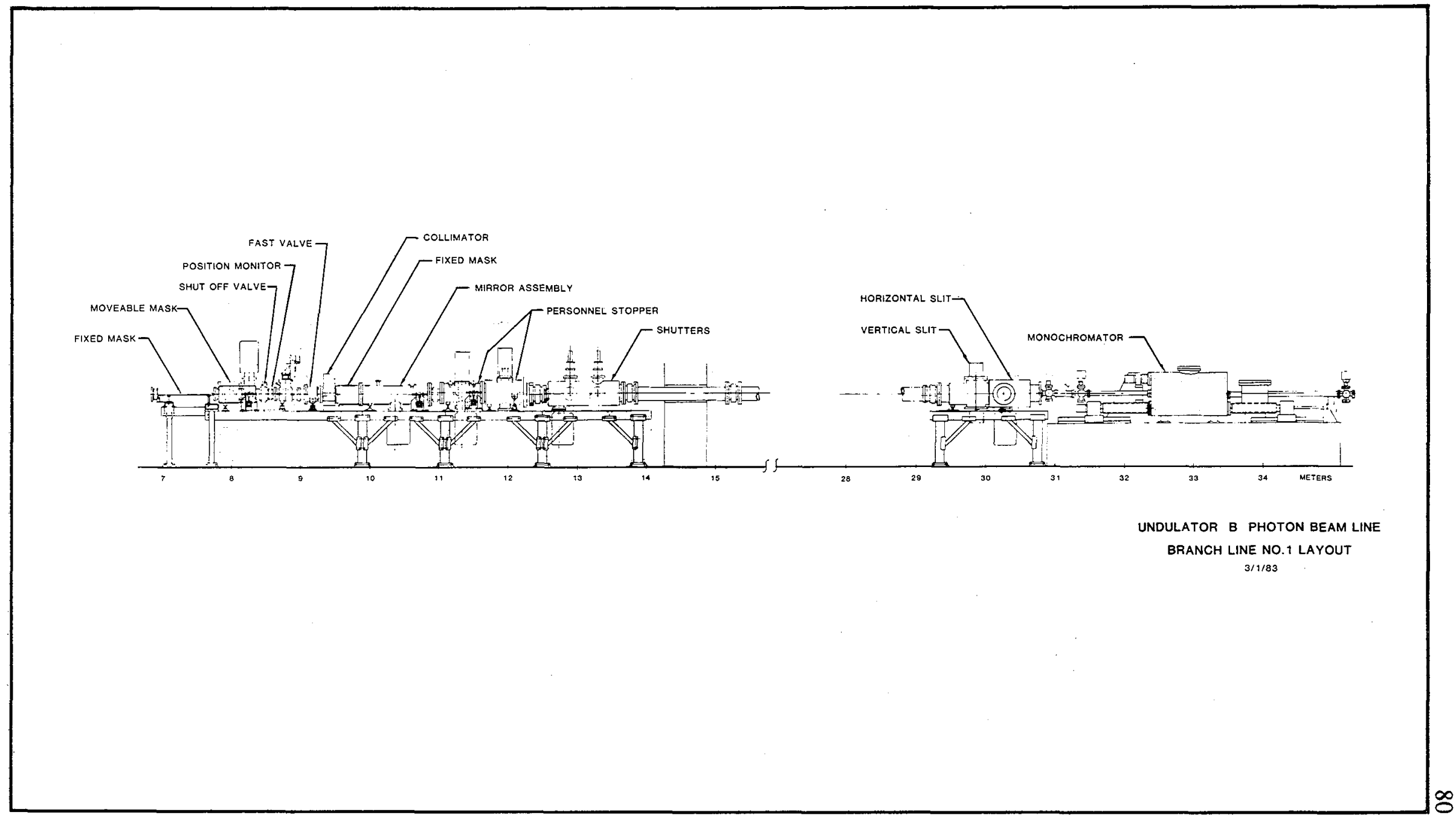

Figure 39. Undulator B photon beamlines, elevation view. 


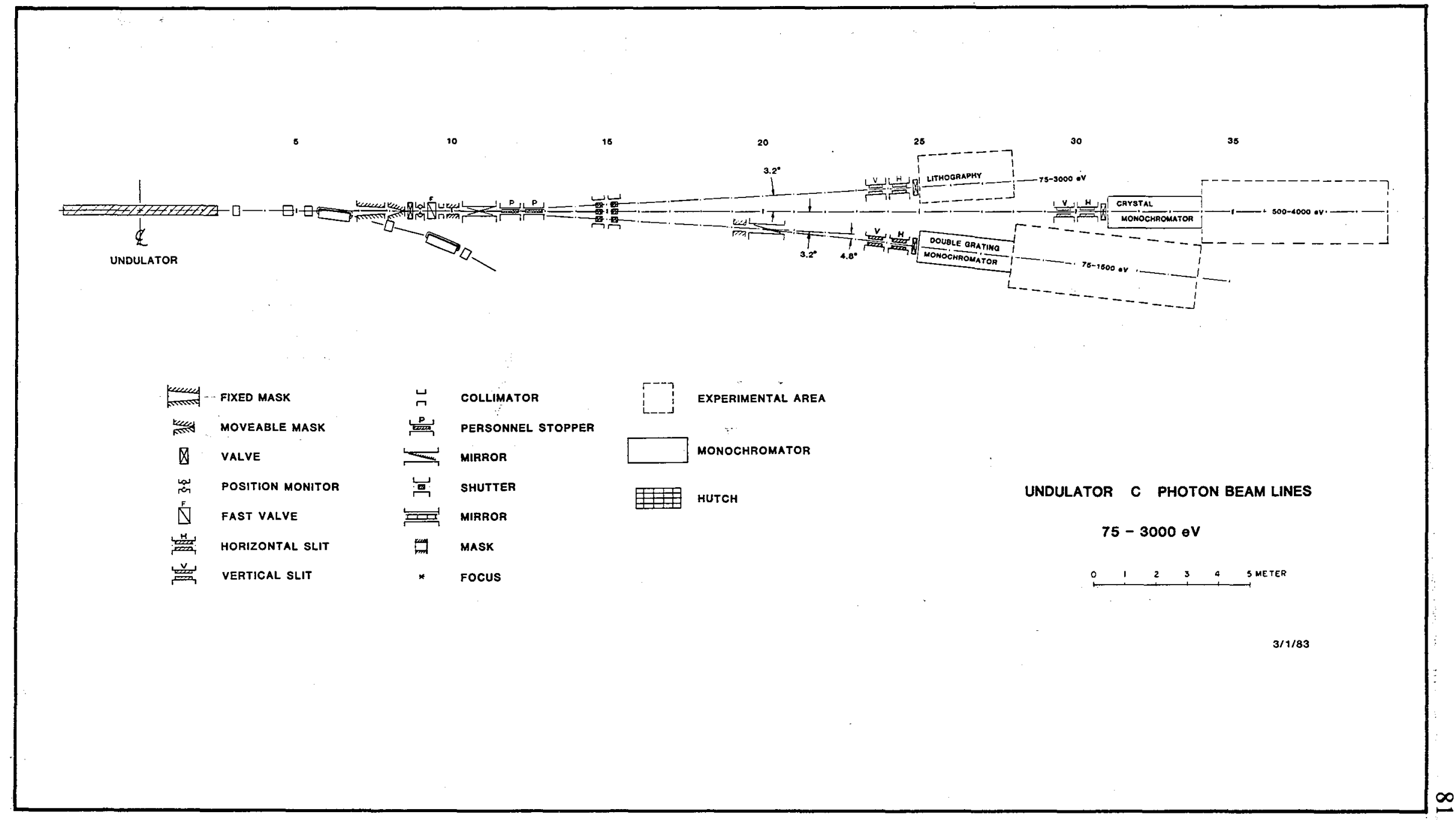

Figure 40. Undulator $C$ photon beamlines. 


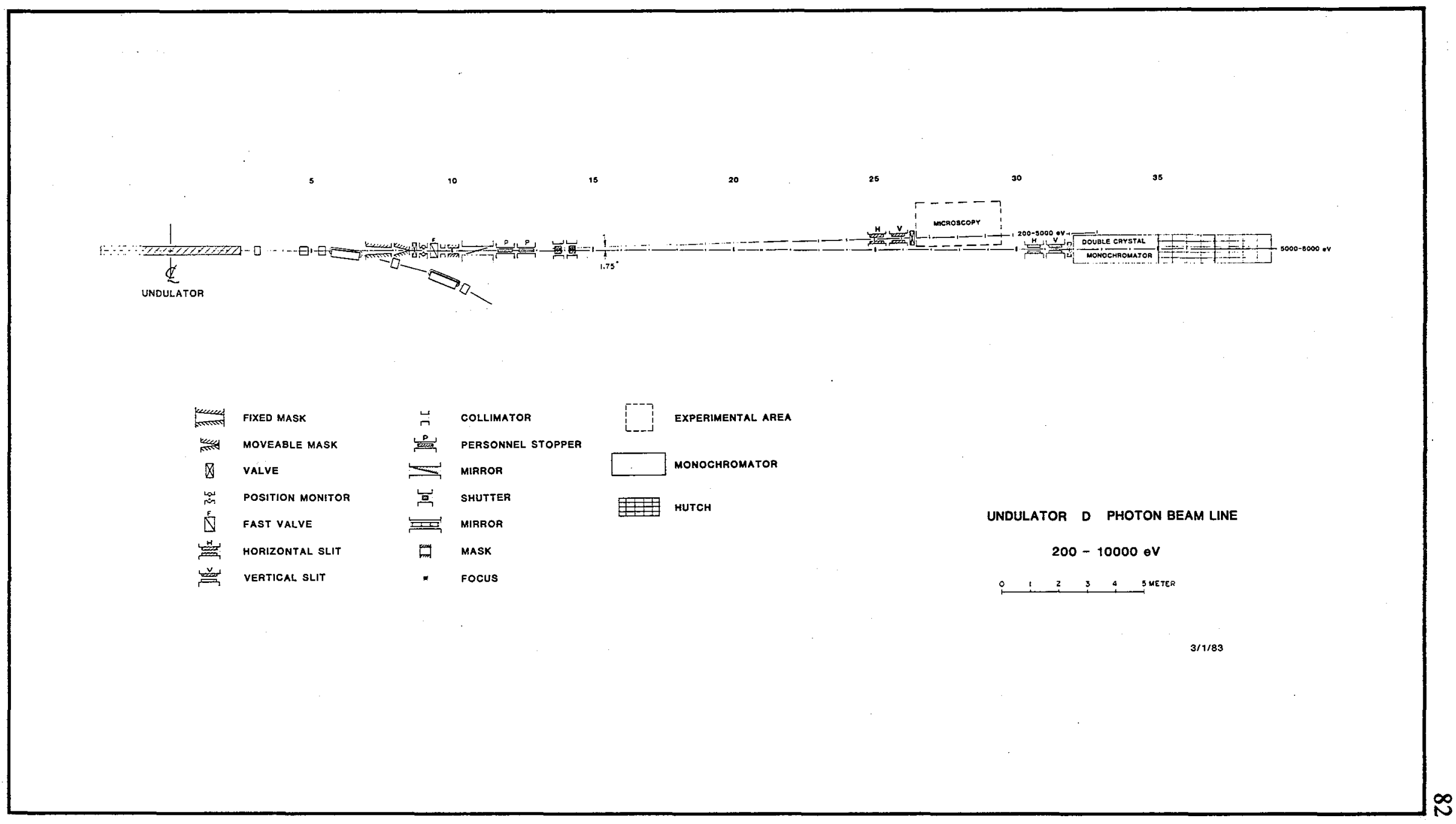

Figure 41. Undulator $D$ photon beamlines. 


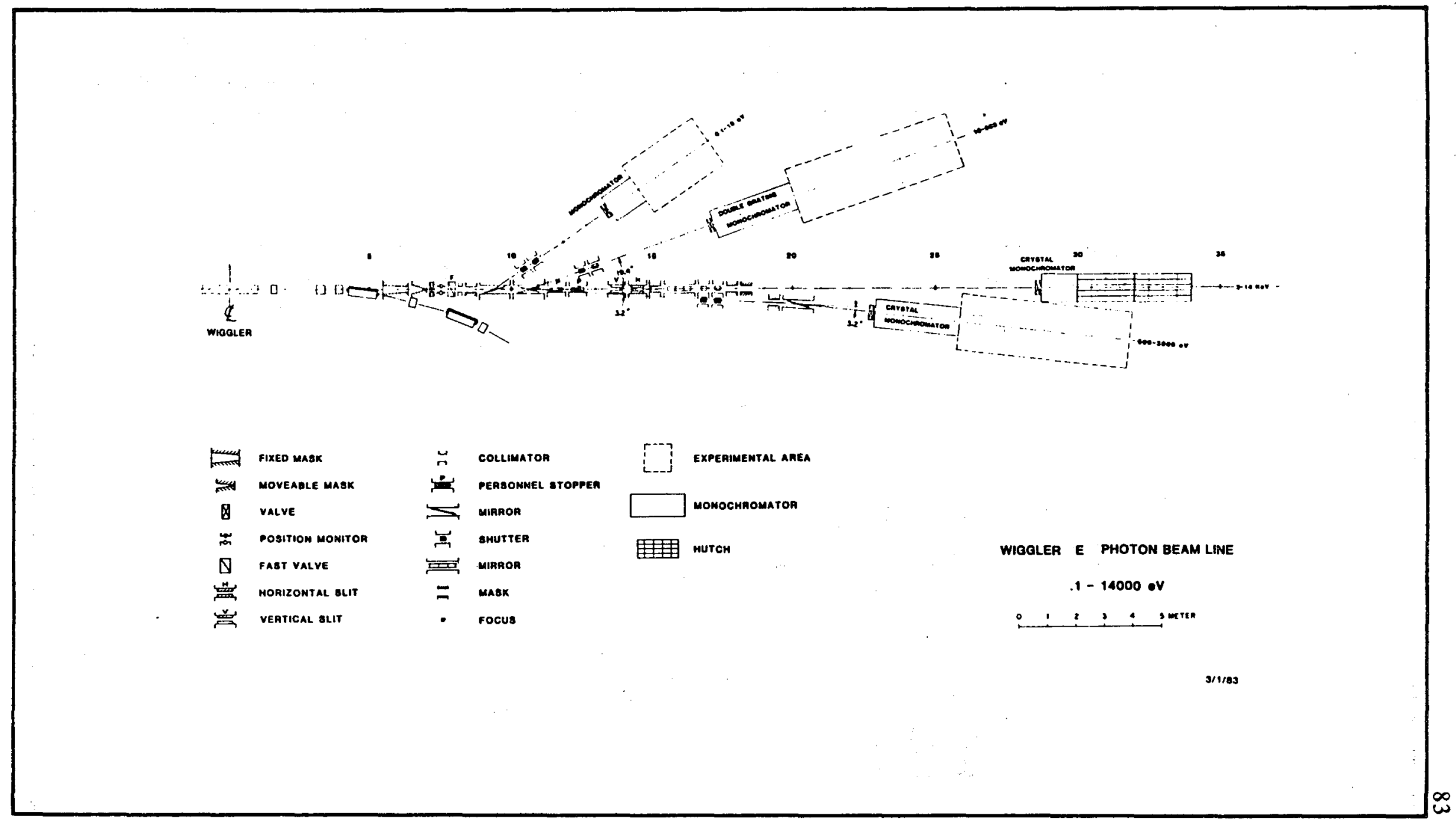

Figure 42. Wiggler $E$ photon beamlines.

XBL 8211-3491A 


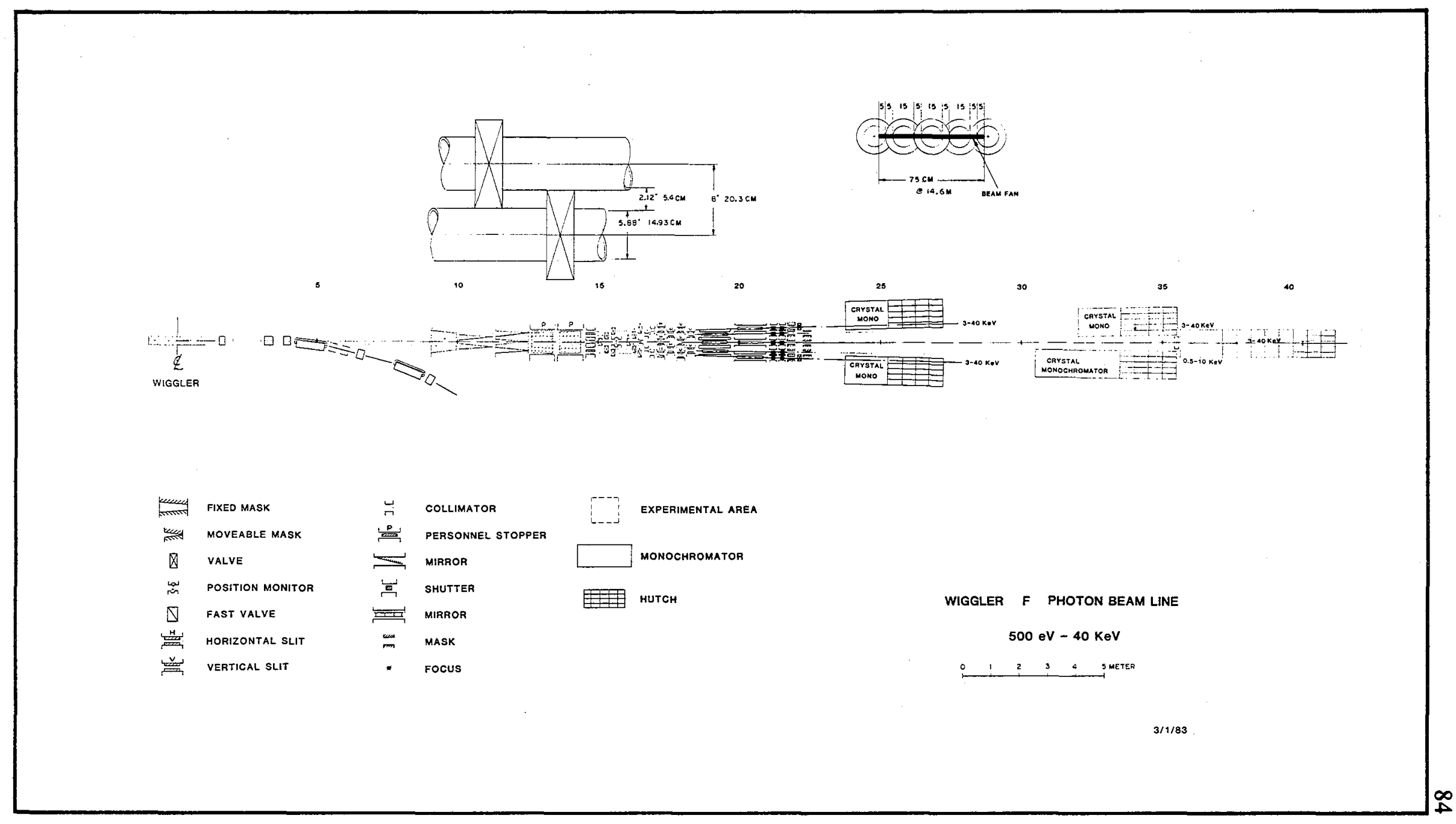

Figure 43. Wiggler $F$ photon beamlines.

XBL 8211-3493A 
In what follows, possible configurations for the six photon beam lines are presented. These are meant to be illustrative and should not be viewed as final layouts.

Undulator beam line $\mathrm{U}_{\mathrm{A}}$ provides very bright photon fluxes in the range $8-400 \mathrm{eV}$. Two experimental stations are envisioned for this line. The straight-through line does not suffer a loss of flux and is equipped with a very-high-resolution monochromator, such as a double plane-grating monochromator.

Photons reach the secondary station by means of a retractable mirror at the $10-\mathrm{m}$ point that deflects the beam by $6^{\circ}$. The monochromator for the secondary station can achieve a high resolution but also is designed for high throughput running.

A large user demand is anticipated for extremely bright photon beams in the energy range from 75-3000 eV. Therefore, two identical beam lines, $U_{B}$ and $U_{C}$, are provided. Three experimental stations are available for each of the $U_{B}$ and $U_{C}$ beam lines: two with monochromators and one "white" beam port for lithography experiments, for example. The main line (undeflected) has a double-crystal monochromator of the JUMBO design which covers the energy range $500-3000 \mathrm{eV}$.

A mirror that deflects the beam by $3.2^{\circ}$ directs the photons to the monochromatic secondary station. The monochromator is a plane-grating type, probably using two gratings for a higher resolution, and covers the $75-1500 \mathrm{eV}$ range. If feasible, an in-vacuum grating/crystal interchanger will be installed to increase the energy range to $3000 \mathrm{eV}$. It is expected that the latter devices will appear on other beam lines as the work horses in this energy range.

The $U_{D}$ beam line has an energy range from 200 to $10000 \mathrm{eV}$ and has the highest brightness of all the ALS beam lines. The main beam line here has a double-crystal, invacuum $\mathrm{x}$-ray monochromator. It is also possible to operate this beam line without the monochromator to give higher intensity and lower energy resolution.

A secondary station is provided here for experiments that can use the high $x$-ray brightness without an $\mathrm{x}$-ray monochromator. Potential experiments on this line are x-ray microscopy and lithography. A mirror is inserted into the x-ray beam at $13 \mathrm{~m}$ to deflect beam into the secondary line. This mirror can either be completely inserted for full intensity or be partially inserted to give useful beam in both beam lines.

The beam lines from the $\mathrm{W}_{\mathrm{E}}$ insertion device are designed to use the smooth energy distribution of flux from the wiggler magnet. Experiments that scan a large energy range, such as Extended X-Ray Absorption Fine Structure (EXAFS) experiments, can readily use this line. In general, several experiments can run simultaneously with different horizontal sections of the beam.

The main beam line, with a horizontal angle of $12 \mathrm{mr}$, is an x-ray line that covers the energy range 3 to $10 \mathrm{keV}$. It is equipped with an $\mathrm{x}$-ray mirror at $14 \mathrm{~m}$ to focus and reduce the harmonic content of the beam. This line has an in-vacuum, double-crystal monochromator to resolve the incident white radiation to an energy resolution, $\Delta \mathrm{E} / \mathrm{E}$, of about $4 \times$ $10^{-4}$. Experiments can be done in either of two $1.5-\mathrm{m}$ by $2-\mathrm{m}$ radiation hutches.

A second beam line, with a horizontal angle of $3 \mathrm{mr}$, is a soft $\mathrm{x}$-ray beam line that covers the energy range 500-3000 eV. The monochromator on this line will be similar to that provided for soft $x$-rays on the undulator beam lines. Experiments on this line, in general, would be scanning the monochromator over a large energy range and would want the flux to vary smoothly over the complete scan. 
A third beam line is provided to cover the energy range 0.1 to $10 \mathrm{eV}$. This line is provided with a mirror and radiation shutters. In this energy range, normal-incidence optics are possible, and there exists a class of good monochromators, e.g., Seya-Namioka.

A fourth beam line is provided for experiments in the 10 to $500 \mathrm{eV}$ range. A highresolution monochromator of the plane-grating type will serve here.

The superconducting wiggler insertion device (described earlier) on beam line $\mathrm{W}_{\mathrm{F}}$ provides an intense beam of photons with an energy range up to $40 \mathrm{keV}$. The horizontal angle is $51 \mathrm{mr}$. This line can accommodate up to five secondary beam lines that each take a portion of the wide incident beam. Each beam line has adjustable horizontal and vertical slits for control of beam size and separate beam stoppers for independent operation.

Three similar beam lines use the central part of the beam. Each has a horizontal acceptance of $10 \mathrm{mr}$ and is equipped with an x-ray mirror and an in-vacuum, doublecrystal monochromator. One of the three central lines is designed as a soft $\mathrm{x}$-ray beam line and, therefore, has a more complex monochromator to cover the energy range from 500 to $10000 \mathrm{eV}$. The other two central lines cover the energy range from 3 to $40 \mathrm{keV}$; they can be operated with the $\mathrm{x}$-ray mirror up to around $30 \mathrm{keV}$ or without the mirror, to above $40 \mathrm{keV}$. For special experiments, it is also possible to operate without the x-ray monochromator or mirror to provide an intense, wide $(40 \mathrm{~cm}$ at $40 \mathrm{~m})$ white radiation beam.

Two secondary beam lines for EXAFS experiments can use the edges of the main beam. These lines have an energy range of 3 to $40 \mathrm{keV}$, and they are also equipped with an $\mathrm{x}$-ray mirror to reduce the harmonic content of the beam. The $\mathrm{x}$-ray monochromator on these lines could be a conventional double-crystal monochromator operating in helium.

So far, we have emphasized the usage of photons from periodic insertion devices. However, valuable photon fluxes can also be collected from the bending magnets around the ring, and they will undoubtedly be utilized in some future beam lines. In the far infrared regions from $.001 \mathrm{eV}$ to $1 \mathrm{eV}$, the bending magnets are especially attractive since the performance of the undulators and wigglers becomes poor in this energy range because of destructive interference.

In an infrared beam line, a large solid angle of photons is collected by an aspheric mirror. The beam is focused at a downstream window assembly and the desired spectrum is transmitted via relay optics to either a Fourier Transform Interference Spectrometer or a diffraction grating spectrometer. It is desirable to operate the line in conjunction with an insertion device beam line, since the intense photons from the latter could be used to excite the samples to be studied. 


\section{References}

1. Coî́sson, R., S. Guiducci, and M.A. Preger, Nuc. Inst. and Meth. 201, 3 (1982).

2. Courant, E.D., M.S. Livingston, and H. Snyder, Phys. Rev. 88, 1190 (1952).

3. Courant, E.D., and H.S. Snyder, Ann. Phys. 3, 1 (1958).

4. Pellegrini, C., "Single Beam Coherent Instabilities in Circular Accelerators and Storage Rings," A.I.P. Conference proceedings, 87, (1982).

5. Laclare, J.L., "Bunched Beam Instabilities," Proceedings of the XIth International Conference on High Energy Accelerators, Geneva (1980).

6. Nodvick, J.S., and P.S. Saxon, Phys. Rev. 96, 180 (1954).

7. Wilson, P., "Ring Impedance and Stored Current for the Photon Factory," KEK-79-20 (Dec. 1979).

8. Bernardini, C., G.F. Corazza, G. Di Giugno, G. Chigo, J. Haissinski, P. Marin, R. Querzoli, and B. Touschek, Phys. Rev. Lett. 10, 407 (1963).

9. Piwinski, A., "Intrabeam Scattering," Proceedings of the IXth International Conference on High Energy Accelerators, Stanford (1974).

10. Wiedemann, H., PATRICIA Code, 1977.

11. Biagini, M.E., S. Guiducci, M. Preger, M. Serio, and S. Tazzari, "Observation of Ion Trapping at ADONE," Proceedings of the XIth International Conference on High Energy Accelerators, Geneva (1980).

12. Brianti, G., Y. Bacconier, O. Gröbner, E. Jones, D. Potaux, and H. Schöneauer, "Accumulation of Ions," Workshop on PP in the SPS, CERN-SPS-PP-1, (Geneva, 1980).

13. "Stanford Linear Collider Conceptual Design Report," SLAC-229 (June 1980). 

This report was done with support from the Department of Energy. Any conclusions or opinions expressed in this report represent solely those of the author(s) and not necessarily those of The Regents of the University of California, the Lawrence Berkeley Laboratory or the Department of Energy.

Reference to a company or product name does not imply approval or recommendation of the product by the University of California or the U.S. Department of Energy to the exclusion of others that may be suitable. 